\title{
Use of Multiplied, Added, Subtracted and/or FiTted Inversion Recovery (MASTIR) pulse sequences
}

\author{
Ya-Jun Ma ${ }^{1}$, Shujuan Fan $^{1}$, Hongda Shao ${ }^{1}$, Jiang Du ${ }^{1}$, Nikolaus M. Szeverenyi ${ }^{1}$, Ian R. Young ${ }^{2 *}$, \\ Graeme M. Bydder ${ }^{1}$ \\ ${ }^{1}$ Department of Radiology, University of California, San Diego, San Diego, CA, USA; ${ }^{2}$ Formerly Department of Electrical Engineering, Imperial \\ College, London, UK \\ Correspondence to: Graeme M. Bydder. Department of Radiology, University of California, San Diego, San Diego CA 92103-8226, USA. \\ Email: gbydder@health.ucsd.edu.
}

\begin{abstract}
The group of Multiplied, Added, Subtracted and/or fiTted Inversion Recovery (MASTIR) pulse sequences in which usually two or more inversion recovery (IR) images of different types are combined is described, and uses for this type of sequence are outlined. IR sequences of different types can be multiplied, added, subtracted, and/or fitted together to produce variants of the MASTIR sequence. The sequences provide a range of options for increasing image contrast, demonstrating specific tissues and fluids of interest, and suppressing unwanted signals. A formalism using the concept of pulse sequences as tissue property filters is used to explain the signal, contrast and weighting of the pulse sequences with both univariate and multivariate filter models. Subtraction of one magnitude reconstructed IR image from another with a shorter TI can produce very high $\mathrm{T}_{1}$ dependent positive contrast from small increases in $\mathrm{T}_{1}$. The reverse subtracted IR sequence can provide high positive contrast enhancement with gadolinium chelates and iron deposition which decrease $T_{1}$. Additional contrast to that arising from increases in $T_{1}$ can be produced by supplementing this with contrast arising from concurrent increases in $\rho_{\mathrm{m}}$ and $T_{2}$, as well as increases or decreases in diffusion using subtraction IR with echo subtraction and/or diffusion subtraction. Phase images may show $180^{\circ}$ differences as a result of rotating into the transverse plane both positive and negative longitudinal magnetization. Phase images with contrast arising in this way, or other ways, can be multiplied by magnitude IR images to increase the contrast of the latter. Magnetization Transfer (MT) and susceptibility can be used with IR sequences to improve contrast. Selective images of white and brown adipose tissue lipid and water components can be produced using different TIs and in and out-of-phase TEs. Selective images of ultrashort and short $T_{2}$ tissue components can be produced by nulling long $T_{2}$ tissue components with an inversion pulse and subtraction of images with longer TEs from images with ultrashort TEs. The Double Echo Sliding IR (DESIRE) sequence provides images with a wide range of TIs from which it is possible to choose values of TI to achieve particular types of tissue and/or fluid contrast (e.g., for subtraction with different TIs, as described above, and for long $\mathrm{T}_{2}$ tissue signal nulling with UTE sequences). Unwanted tissue and fluid signals can be suppressed by addition and subtraction of phase-sensitive (ps) and magnitude reconstructed images. The sequence also offers options for synergistic use of the changes in blood and tissue $\rho_{\mathrm{m}}, \mathrm{T}_{1}, \mathrm{~T}_{2} / \mathrm{T}_{2}{ }^{*}, \mathrm{D}^{*}$ and perfusion that can be seen with fMRI of the brain. In-vivo and ex-vivo illustrative examples of normal brain, cartilage, multiple sclerosis, Alzheimer's disease, and peripheral nerve imaged with different forms of the MASTIR sequence are included.
\end{abstract}

Keywords: Magnetic resonance imaging (MRI); functional MRI; inversion recovery (IR); pulse sequence; MASTIR; image contrast; tissue property filters

*, deceased September 27, 2019. 
Submitted Apr 13, 2020. Accepted for publication Apr 26, 2020.

doi: 10.21037/qims-20-568

View this article at: http://dx.doi.org/10.21037/qims-20-568

\section{Introduction}

The inversion recovery (IR) pulse sequence was used early in the history of magnetic resonance imaging (MRI) because of its high gray white matter contrast in the brain, and its sensitivity to the presence of diseases which produce an increase or decrease in $T_{1}$ (1-3). The IR sequences used in early studies of the brain and other organs of the body employed intermediate (medium) inversion times (TIs) which resulted in the longitudinal magnetization of the tissues of interest being positive at the time that the $90^{\circ}$ radiofrequency (rf) pulse necessary for data collection (dc) was applied (Figure 1).

It was also possible to use short TI IR (STIR) sequences to null the signal from fat (which had the shortest $T_{1}$ of the tissues that could be imaged with detectable signal at this time) (4). Using this sequence, the longitudinal magnetization of other (longer) $\mathrm{T}_{1}$ tissues was negative at the time the $90^{\circ}$ pulse was applied (Figure 2). For lesions displaying the common change in disease of an increase in each of the three tissue properties mobile proton density $\left(\rho_{\mathrm{m}}\right), \mathrm{T}_{1}$ and $\mathrm{T}_{2}$, and using magnitude reconstruction for the IR sequence, this resulted in high synergistic positive contrast from concurrent increases in each of these three tissue properties so that lesions showed high signal relative to normal tissue. In addition to this high signal from lesions, the STIR sequence also showed very high signal from long $\mathrm{T}_{1}$ and $\mathrm{T}_{2}$ fluids such as cerebrospinal fluid (CSF). This was an advantage in some circumstances, but a disadvantage in others because it resulted in reduced display dynamic range for the tissue abnormalities of principal interest, as well as problems due to partial volume effects between very high signal fluids and normal tissue simulating, or obscuring, relatively high signal lesions in tissues.

One solution to this problem was to use successive inversion pulses in the form of the Double IR (DIR) sequence to null not just fat signals but fluid signals as well (4). The effect was that of multiplying two IR sequences with different TIs. In organs where there was no fat such as the brain it was also helpful to employ the DIR sequence with the shorter of the two TIs used to null either white or gray matter signal (rather than fat signal), and the longer TI used to null CSF in order to selectively visualize gray or white matter respectively (5).
Another option with the IR sequence was to specifically invert and null only the fat signal using pulse sequences selectively tuned to the fat resonant frequency so that they did not affect water signals using SPectral IR or Spectral Presaturation IR (SPIR) (6), as well as SPectral Adiabatic IR or SPectral Attenuated IR (SPAIR), and SPECtral Inversion At Lipid (SPECIAL) pulse sequences. These methods of fat signal suppression could be used with a wide variety of pulse sequences. Fast versions of these sequences were often used with partial inversion pulses (e.g., $105^{\circ}$ ) and an ultrashort TI $\left(\mathrm{TI}_{\mathrm{u}}\right)$ (e.g., $10-15 \mathrm{~ms}$ ).

The use of fluid signal suppression with a long TI coupled to a long echo time (TE) to provide heavy $\mathrm{T}_{2}$ weighting was another development which was published in 1992 (7). This FLuid Attenuated IR (FLAIR) (or later $T_{2}-$ FLAIR) sequence has been widely used since that time for imaging the brain and spinal cord. It provides high signal images of diseased tissue without confusion from partial volume effects between very high signal CSF and normal brain simulating lesions in the brain.

These approaches exploited the high $T_{1}$ contrast of IR sequences, suppression of unwanted signals from fat and other tissues such as white and gray matter, as well as suppression of signals from long $\mathrm{T}_{1}$ fluids (particularly CSF) using single inversion pulses. The exception was the DIR sequence $(4,5)$ which multiplied the effects of two inversion pulses together.

The purpose of this paper is to extend the concept of multiplying two IR sequences together to include Multiplying, Adding, Subtracting and/or fiTting them as MASTIR pulse sequences. The IR images or sequences which are combined in these ways may differ in their type of inversion pulse, TI, Magnetization Transfer (MT) pulses, TE, apparent diffusion $\left(\mathrm{D}^{*}\right)$ weighting, type of $\mathrm{dc}$, image reconstruction [phase-sensitive (ps) or magnitude (m)], post-processing and other sequence parameters. Some examples where IR sequences are combined with non-IR sequences are also included.

The objectives of combining sequences include: (i) to develop high $T_{1}$ dependent contrast (including forms of the sequence in which the $T_{1}$ contrast developed by different variants of the sequence combine synergistically); (ii) to obtain additional contrast to that developed from 


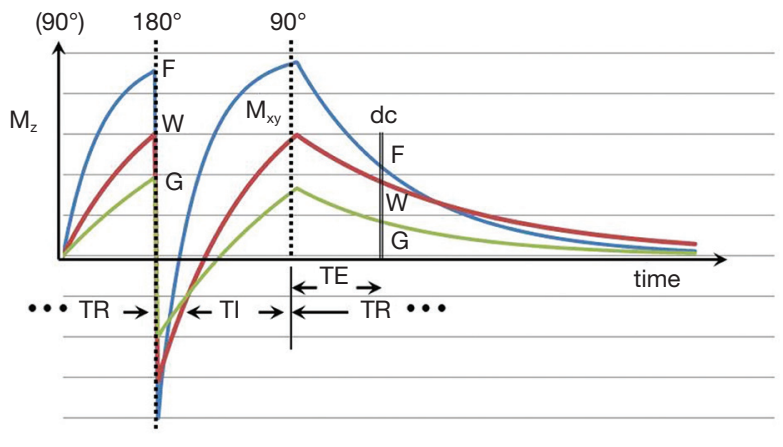

Figure 1 The basic IR pulse sequence with $M_{z}$ then $M_{x y}$ plotted against time. After the initial $90^{\circ}$ pulse from the preceding cycle of the pulse sequence the longitudinal magnetization $M_{z}$ is zero. It recovers with time constant $T_{1}$ and is inverted by the $180^{\circ}$ pulse. $\mathrm{M}_{\mathrm{z}}$ then recovers during TI. When the second $90^{\circ}$ pulse shown is applied $M_{z}$ becomes $M_{x y}$. This decays with time constant $T_{2}$ during $\mathrm{TE}$ and is detected during the data collection (dc). Plots for fat $(\mathrm{F})$, white matter $(\mathrm{W})$ and gray matter $(\mathrm{G})$ are shown. The signal shown on the image is proportional to the magnitude of $M_{x y}$ for $\mathrm{F}, \mathrm{W}$ and $\mathrm{G}$ at the time of the dc. Each cycle of the sequence is described as beginning with a $180^{\circ}$ pulse which is then followed after TI by a $90^{\circ}$ pulse.

$\mathrm{T}_{1}$ differences by supplementing this with contrast due to differences or changes in other tissue properties such as $\rho_{\mathrm{m}}, \mathrm{T}_{2}$, and $\mathrm{D}^{*}$; (iii) to increase the image contrast produced by clinically used contrast agents; (iv) to provide specific imaging of particular tissues or groups of them; (v) to provide options for imaging of blood as well as other fluids such as lymph, urine and CSF, and (vi) to suppress unwanted signals from tissues and/or fluids.

The paper provides a formalism for understanding the use of variants of the MASTIR sequence in these different applications, and includes both established sequences and new variants. It begins with a description of the basic IR sequence, follows this with an outline of the Tissue Property filters (TP-filters) approach to understanding the signal, contrast and weighting of MR images, includes a description of different types of MASTIR sequence, provides illustrative examples, and discusses more general issues. A list of abbreviations used in this paper is included in the Appendix.

\section{The basic IR pulse sequence}

\section{General}

The basic pulse sequence is illustrated in Figure 1. Recovery

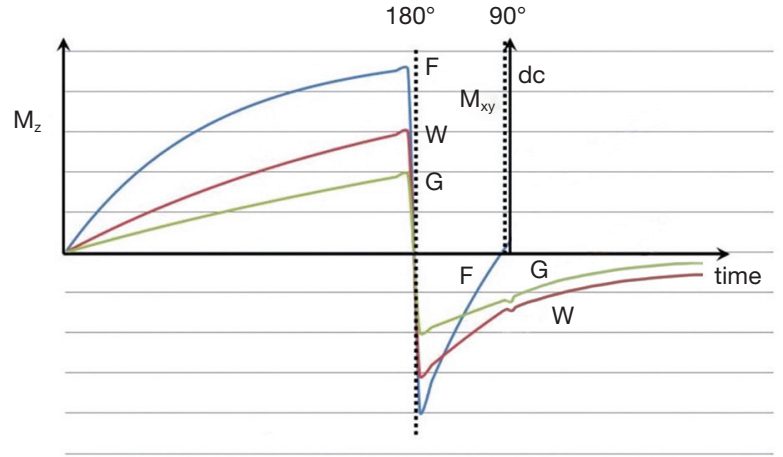

Figure 2 The short TI IR (STIR) sequence. The longitudinal magnetization $M_{z}$ is zero after the first $90^{\circ}$ pulse (from the previous cycle) and recovers with time constant $T_{1}$ for each tissue or fluid. After the $180^{\circ}$ pulse during TI fat (F) recovers rapidly typically to reach zero at the time of the $90^{\circ}$ pulse, but longer $T_{1}$ tissues such as white $(\mathrm{W})$ and gray $(\mathrm{G})$ matter recover more slowly, and their magnetization is negative at the time of the $90^{\circ}$ pulse. $M_{z}$ becomes $M_{x y}$ as a result of the $90^{\circ}$ pulse. During TE it decays with time constant $T_{2}$. W and $G_{2} M_{2}$ are negative at the time of the second $90^{\circ}$ pulse. The effects of an increase in the $T_{1}$ and $T_{2}$ in both $W$ and $G$ are synergistic in producing an increase in signal relative to normal.

of the longitudinal magnetization $\mathrm{M}_{\mathrm{z}}$ occurs during TR after the $90^{\circ}$ pulse of the previous cycle. The inversion $\left(180^{\circ}\right)$ rf pulse then inverts $\left(M_{Z}\right)$. After time TI a $90^{\circ} \mathrm{rf}$ pulse is applied. This converts $M_{Z}$ into transverse magnetization $\left(M_{X Y}\right)$. After a further time TE this magnetization is detected during the dc and the image is reconstructed and processed. The signal detected during the $\mathrm{dc}$ is proportional to $\mathrm{M}_{\mathrm{XY}}$. The magnitude of this depends on: (i) the first $\mathrm{T}_{1}$ dependent preparation period including that from the initial $90^{\circ}$ pulse shown in Figure 1 to the $180^{\circ}$ pulse (part of TR); (ii) the type, and duration of the initial inversion pulse; (iii) the second $T_{1}$ dependent preparation period from the $180^{\circ}$ pulse to the $90^{\circ}$ pulse (TI); (iv) the $\mathrm{T}_{2}$ dependent preparation period from the $90^{\circ}$ pulse to the dc (TE); (v) the type of dc; (vi) the method of image reconstruction; (vii) image processing, and (viii) the repetition time (TR). Each of these is dealt with below.

\section{The first $T_{1}$ dependent preparation time (TR)}

The first $T_{1}$ dependent preparation time is from the initial $90^{\circ}$ pulse shown in Figure 1 to the $180^{\circ}$ pulse. It forms part of the recovery time TR. 
Table $1 T_{1}$ and $T_{2}$ values of tissues and fluids at $1.5 \mathrm{~T}$

\begin{tabular}{|c|c|c|c|c|c|c|}
\hline Tissues and fluids & \multicolumn{3}{|c|}{$\mathrm{T}_{1}(\mathrm{~ms})$} & \multicolumn{3}{|c|}{$\mathrm{T}_{2}(\mathrm{~ms})$} \\
\hline Fat & 240-250 & 200 & 310 & $60-80$ & - & 117 \\
\hline White matter & 780 & 560 & 1,000 & 90 & 82 & 112 \\
\hline Gray matter & 920 & 1,100 & 1,260 & 100 & 92 & 109 \\
\hline Muscle & $860-900$ & 1,075 & 980 & 50 & 33 & 36 \\
\hline Blood (deoxygenated) & 1,350 & - & - & 50 & - & - \\
\hline Blood (oxygenated) & 1,350 & - & 1390 & 200 & - & 310 \\
\hline CSF & $4,200-4,500$ & 2,060 & 4,630 & $2,100-2,300$ & - & - \\
\hline
\end{tabular}

CSF, cerebrospinal fluid.

In Figure 2 the recovery of longitudinal magnetization depends on $T_{1}$ which is shortest for fat $(F)$, followed by white matter $(W)$ and then gray matter $(G)$. Addition preparation pulses may be applied during this period.

\section{The initial inversion pulse}

There are many types of inversion pulse but the two general groups of interest in this context are "hard" (or short) pulses and "soft" (or long) pulses. The first group of hard pulses are of very short duration compared with the $\mathrm{T}_{2} \mathrm{~S}$ of the tissues of interest and may only last a few microseconds. They can fully, or almost fully, invert all the longitudinal magnetization without significant transverse relaxation occurring while the pulse is being applied. After their application, ultrashort $T_{2}$ tissue components $\left(T_{2} \approx 0.1\right.$ to $1 \mathrm{~ms}$ ) are typically fully inverted. Hard pulses require high rf peak power and are available on small bore NMR spectrometers but not usually on clinical machines.

The second group is soft pulses. Their duration (e.g., 1-20 ms) is of the same order, or longer than the shortest $\mathrm{T}_{2} \mathrm{~s}$ of detectable tissue. While the longitudinal magnetization of long $T_{2}$ tissues or fluids is fully inverted by these pulses, ultrashort $T_{2}$ tissues undergo transverse relaxation during the time that the inversion pulse is applied and are not fully inverted. As a result, their longitudinal magnetization is partially saturated, saturated and/or partially inverted. Soft pulses are the type of inversion pulse commonly used on clinical MR machines.

\section{The second $T_{1}$ dependent preparation time (TI)}

The recovery of $M_{Z}$ after inversion of the longer $T_{2}$ components of fat $(\mathrm{F})$, white matter $(\mathrm{W})$, and gray matter $(\mathrm{G})$, are shown in Figure 1. Shorter $\mathrm{T}_{1}$ tissues recover more quickly than longer $\mathrm{T}_{1}$ fluids (such as blood and CSF). Published values of $T_{1}$ for these tissues are given in Table 1 . These values increase with static field strength $\left(\mathrm{B}_{\mathrm{O}}\right)$.

Addition rf pulses may be applied during this period to achieve fat signal suppression or "fat saturation". Magnetization preparations such as MT and $\mathrm{T}_{2}$-preparation can also be applied during this time, as described below.

\section{Fat saturation}

It is possible to apply spectrally selective pulses that only affect fat which has a chemically shifted major $-\mathrm{CH}_{2}$ peak at a resonant frequency $3.3 \mathrm{ppm}$ lower than that of water. The fat longitudinal magnetization can be selectively rotated through $90^{\circ}$ and then dephased with gradients [chemical shift selective saturation, CHESS (11)] to achieve saturation.

The term "fat saturation" is also applied to the situation where an inversion pulse is applied with inversion time $\mathrm{TI}_{\text {WATN }}$ (TI white adipose tissue nulling) chosen to selectively null white fat or white adipose tissue (WAT) without affecting the water longitudinal magnetization. To save time the "inversion" pulse which is applied is often only a partial inversion in the sense that the longitudinal magnetization is rotated beyond $90^{\circ}$ (e.g., to $105^{\circ}$ ), but not to $180^{\circ}$. This can then be coupled with an ultrashort TI, $\mathrm{TI}_{\mathrm{u}}$ or $\mathrm{TI}_{\text {WATN }}$ (e.g., $5-10 \mathrm{~ms}$ ) to produce selective nulling of 
Table 2 Types of data collections (dcs) used with the IR sequence

Gradient echo (GE) and Rapid gradient echo (RAGE)

Spin echo (SE and Fast Spin Echo)

Ultrashort TE (UTE) and Zero TE (ZTE)

Echo planar imaging (EPI)

Balanced steady state free precession (bSSFP)

Dixon in-phase (IP) and out-of-phase (OP) GE or Asymmetric SE (ASE)

Interaction Decomposition of Water and Fat with Echo Asymmetry and Least Squares Estimation (IDEAL)

the WAT signal in a very short time.

\section{Magnetization transfer (MT)}

MT off-resonance pulses used with IR sequences can be applied during both $T_{1}$ dependent preparation periods i.e., before the $180^{\circ}$ pulse during TR, or after the $180^{\circ}$ pulse during TI, as well as during both periods. The pulses reduce both the detectable magnetization or observed $\rho_{\mathrm{m}}$, and the observed $T_{1}$ of the detected free pool, and generally do this more for normal than abnormal tissues i.e., the latter show a reduced MT effect. As a result, when MT pulses are used abnormal tissue can appear to have an increased observed $\rho_{\mathrm{m}}$ and an increased observed $\mathrm{T}_{1}$ relative to normal tissue.

\section{$\mathrm{T}_{2}$-preparation}

This consists of an excitation $90^{\circ}$ pulse, $180^{\circ}$ refocussing pulse and a negative $90^{\circ}$ pulse. It can be used to reduce the longitudinal magnetization to zero prior to the inversion pulse and so lead to more recovery of it during TI.

\section{The $T_{2}$ dependent preparation time (TE)}

After the second $90^{\circ}$ pulse shown in Figure 1 the transverse magnetization $\left(M_{\mathrm{XY}}\right)$ decays with time constant $\mathrm{T}_{2}$ which is different for each tissue. In general, tissues with long $\mathrm{T}_{1} \mathrm{~s}$ have long $\mathrm{T}_{2} \mathrm{~s}$ so that the contrast developed as a result of differences in longitudinal magnetization recovery during $\mathrm{TI}$ is often reversed during TE i.e., the $\mathrm{T}_{1}$ dependent contrast developed in either or both of the $T_{1}$ dependent preparation times is opposed by the $T_{2}$ dependent contrast developed during the $T_{2}$ dependent preparation time. This applies to lesions which have an increase in both $T_{1}$ and $T_{2}$ relative to normal tissue. However, with a short TI the $\mathrm{T}_{1}$ dependent contrast developed in the second $\mathrm{T}_{1}$ dependent preparation period TI, is synergistic with the $T_{2}$ contrast developed in the $T_{2}$ dependent preparation period TE for lesions with an increase in $T_{1}$ and $T_{2}$. This increases lesion contrast (Figure 2).

Additional contrast may be produced during TE from susceptibility effects and diffusion weighting as described below.

\section{Susceptibility}

In the presence of inhomogeneous local magnetic fields due to differences in the static field $\mathrm{B}_{0}$ or tissue susceptibility $\mathrm{T}_{2}$ decay occurs more rapidly than in a homogeneous field and this is denoted by a shorter value for $T_{2}$, the apparent $\mathrm{T}_{2}\left(\mathrm{~T}_{2}{ }^{*}\right)$. This is the situation when gradient echo (GE) acquisitions are used. The additional loss of signal with GE sequences provides sensitivity to susceptibility differences. The effect is reversed by spin echo (SE) sequences.

\section{Diffusion weighting}

Additional gradient magnetic fields can be applied during $\mathrm{TE}$, before and after the $180^{\circ}$ pulse of a SE sequence to produce diffusion weighted contrast as the Stejskal-Tanner Pulsed Gradient SE (PGSE) pulse sequence.

\section{The data collection (dc)}

A list of techniques for dc is shown in Table 2. They include SE and Fast SE (FSE), GE, Rapid Acquisition Gradient Echo (RAGE), balanced Steady State Free Precession (bSSFP), and echo planar imaging (EPI).

There is also a class of fat water suppression or separation (FWSS) techniques such as Dixon (DX) [addition and subtraction of in-phase (IP) and out-of-phase (OP) images acquired at different TEs to provide separate water and fat images respectively] (Table 3).

Ultrashort TE (UTE) and Zero TE (ZTE) are examples of another class of des which utilize very short TEs to detect signal from ultrashort $T_{2}$ tissues. Signal from these 
Table 3 Fat Signal Suppression and Separation (FWSS) techniques

ASE

SE

GE

IDEAL

Selective water excitation

Subtraction (Diffusion based)

Subtraction (MT based)

tissues or tissue components is not usually detected with conventional longer TE GE or SE sequences.

\section{Image reconstruction}

Phase-sensitive (ps) and magnitude $(\mathrm{m})$ reconstruction Forms of image reconstruction of interest in this context are ps and m. As shown in Figures 1,2 the longitudinal magnetization may be positive or negative at TI when the $90^{\circ}$ pulse is applied. ps reconstruction takes this into account and images of the type have both positive and negative signal values. Since the display only shows positive levels of brightness, negative values of signal are shown as low intensity on the display brightness scale i.e., low or zero brightness. Zero image signal level with the sequence then appears mid-gray on the display (corresponding to the value for zero signal seen with no signal and only noise around the outside of the image), and positive sequence signals appear lighter than this.

In the other option, $m$ reconstruction, the signal amplitude of the image is shown irrespective of the sign of its magnetization. Positive magnetization appears the same with both $\mathrm{m}$ and $\mathrm{ps}$ reconstruction, but with $\mathrm{m}$ reconstruction the negative magnetization seen with ps reconstruction is "reflected" across the $\mathrm{X}$ axis and appears positive. Between the positive and negative longitudinal magnetizations there may be a cancellation line where the signal in voxels is zero because the positive magnetization in the voxel cancels out the negative magnetization in it. This may occur with partial volume effects between tissues or fluids with positive and negative longitudinal magnetizations respectively at the time of the dc. Since there are no negative signals with $\mathrm{m}$ reconstruction the display shows all signals with brightness monotonically increasing with signal level from zero. The surrounding noise level on $\mathrm{m}$ reconstructed images is zero and corresponds to zero display signal.

\section{Phase mapping}

In addition to the images using the amplitude of the signal as described above, there are phase maps showing the phase of the vector transverse magnetization at the time of the dc. There is confusion between the use of the term phase (as in phase-sensitive reconstruction) where it is used to designate image reconstruction which shows both positive and negative magnetization, and the use of phase in the sense of an angle describing a vector (such as transverse magnetization) in polar coordinates. Although most imaging is performed with images based on the amplitude of the transverse magnetization vector, phase maps showing the angle of the voxel transverse magnetization at the time of signal detection can also be displayed and provide distinct information.

\section{Image processing}

The particular forms of image processing for this purpose are multiplication, addition, subtraction and/or fitting of one IR image with, or to another image.

\section{The repetition time (TR)}

This includes the time from the initial $90^{\circ}$ pulse until the $180^{\circ}$ pulse (Figure 1 ) as well as the time after the end of the dc to the first $90^{\circ}$ pulse. TR is the time between different cycles of the sequence (i.e., the time between the repetition of each rf pulse).

The recovery time available for the longitudinal magnetization to recover during TR is shorter than this. It may be further shortened by the use of $180^{\circ}$ pulses in a SE or FSE dc. Recovery of longitudinal magnetization also occurs during TI.

\section{Pulse sequences as tissue property filters (TP- filters) (12)}

The common way of showing the signal and contrast between two tissues or fluids with different $T_{1} s$ and $T_{2} s$ using IR and SE sequences is to plot $M_{Z}$ against time beginning with a $90^{\circ}$ pulse (when $M_{Z}$ is zero), and after the $90^{\circ}$ pulse necessary for the dc, follow this with a plot of $M_{X Y}$ against time (since $M_{Z}$ becomes $M_{X Y}$ as a result of the $90^{\circ}$ pulse). This is shown for the IR sequences in Figures 1 and 2, and a SE sequence in Figure 3. Detected signal is 


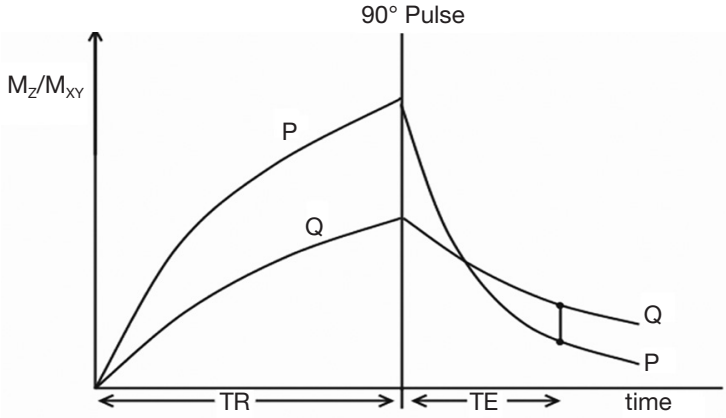

Figure 3 Composite diagram for the SE sequence. This shows recovery of the longitudinal magnetization $\left(M_{z}\right)$ for time TR, followed by decay of the transfer magnetization $\left(M_{x y}\right)$ for time TE after the $90^{\circ}$ pulse, for two tissues, $\mathrm{P}$ and $\mathrm{Q}$. The contrast between the two tissues is proportional to the difference between the two curves (vertical line) at time TE during the dc.

represented by the height of $M_{X Y}$ at the time (TE) of the dc and contrast is represented by the difference in $M_{X Y}$ for $F, W$ and $\mathrm{G}$ in Figures 1 and 2, and that between two tissues $\mathrm{P}$ and $\mathrm{Q}$ in Figure 3. This is useful, but becomes complicated with the use of ps and $\mathrm{m}$ reconstructions as well as additions or subtractions, and it does not include the contributions of $\rho_{\mathrm{m}}$, $\mathrm{D}^{*}$ or other tissue properties to voxel signal and contrast.

An alternative is to start with the simplified Bloch equations, e.g., for a SE sequence where the signal from a voxel $\mathrm{S}$ is given by

$$
\mathrm{S}=\mathrm{K} \rho_{\mathrm{m}}\left(1-\mathrm{e}^{-\mathrm{TR} / \mathrm{T} 1}\right) \mathrm{e}^{-\mathrm{TE} / \mathrm{T} 2}
$$

where $\rho_{\mathrm{m}}, \mathrm{TR}$, and TE have their usual meanings and $\mathrm{K}$ is a scaling function.

It is possible to represent this equation as the multiplication of three segments $S \rho_{\mathrm{m}}, \mathrm{S}_{\mathrm{T} 1}$, and $\mathrm{S}_{\mathrm{T} 2}$ so

$$
\mathrm{S}=\mathrm{KS} \rho_{\mathrm{m}} \mathrm{S}_{\mathrm{T} 1} \mathrm{~S}_{\mathrm{T} 2}
$$

where

$$
\mathrm{S} \rho_{\mathrm{m}}=\rho_{\mathrm{m}}, \mathrm{S}_{\mathrm{T} 1}=\left(1-\mathrm{e}^{-\mathrm{TR} / \mathrm{T} 1}\right) \text {, and } \mathrm{S}_{\mathrm{T} 2}=\mathrm{e}^{-\mathrm{TE} / 2}
$$

In Figures 1-3 magnetization is plotted against time. However, for any given IR or SE pulse sequence the times TR, TI and TE are fixed, while the tissue properties $\rho_{\mathrm{m}}, \mathrm{T}_{1}$, and $T_{2}$ vary, and often cover a wide range of values. For the $\mathrm{SE}$ sequence the equations for time dependent change of $\mathrm{S}$ during TR and TE respectively are of the form $y=1-e^{-x}$, and $\mathrm{y}=\mathrm{e}^{-\mathrm{x}}$ for $\mathrm{T}_{1}$ and $\mathrm{T}_{2}$ respectively as shown in Figure 4 , whereas those for change in the tissue properties $T_{1}$ or $T_{2}$ at the fixed times TR and TE of the SE sequence are of the

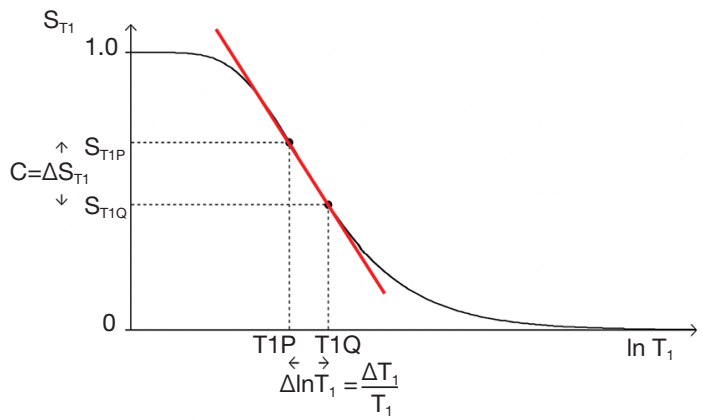

Figure 4 Relationship between change in $\ln T_{1}\left(\Delta \ln T_{1}\right)$ and change in signal (or contrast) $\Delta \mathrm{S}_{\mathrm{T} 1}$. The positive difference $\Delta \ln \mathrm{T}_{1}=\Delta \mathrm{T}_{1} / \mathrm{T}_{1}$ from $\mathrm{P}$ to $\mathrm{Q}$ along the $\mathrm{X}$ axis produces a negative change in signal $\Delta S_{\mathrm{T} 1}$ from $\mathrm{P}$ to $\mathrm{Q}$ along the $\mathrm{Y}$ axis in a region where the $\mathrm{T}_{1}$-filter is steeply sloping. The change $\Delta \ln T_{1}$ would produce little or no signal contrast if it was opposite either the high or the low signal plateau regions of the filter. The slope of the curve between $\mathrm{P}$ and $\mathrm{Q}$ which is the $T_{1}$-filter sequence weighting is shown in red. It is negative.

quite different forms $\mathrm{y}=1-\mathrm{e}^{-1 / x}$, and $\mathrm{y}=\mathrm{e}^{-1 / x}$ respectively as shown in Figures 4,5 using a natural logarithmic (ln) $\mathrm{X}$ axis.

$\mathrm{S}_{\mathrm{T} 1}$ is a low pass filter (Figure 4) and $\mathrm{S}_{\mathrm{T} 2}$ is a high pass filter (Figure 5). By this it is meant that lower (shorter) values of $T_{1}$ "pass" (i.e., they are not reduced) with the $T_{1}$ filter, and higher (longer) values of $T_{2}$ pass with the $T_{2}$ filter. The curves also show that an increase in $T_{1}$ from $P$ to $\mathrm{Q}$ decreases signal shown along the $\mathrm{Y}\left(\mathrm{S}_{\mathrm{T} 1}\right)$ axis in Figure 4 , and that an increase in $T_{2}$ from $P$ to $Q$ increases signal shown along the $\mathrm{Y}\left(\mathrm{S}_{\mathrm{T} 2}\right)$ axis in Figure 5 . Thus, the contrast $\mathrm{C}=\Delta \mathrm{S}_{\mathrm{T} 1}$ (difference in signal) produced by an increase in $\mathrm{T}_{1}$ is negative, and the contrast $\Delta \mathrm{S}_{\mathrm{T} 2}$ produced by an increase in $T_{2}$ is positive.

With an increase in TR the curve for $\mathrm{S}_{\mathrm{T} 1}$ in Figure 4 shifts to the right and the negative slope of the curve decreases. With an increase in TE the curve for $\mathrm{S}_{\mathrm{T} 2}$ shown in Figure 5 also shifts to the right and its positive slope decreases.

The curves in Figures 4,5 both have a high and low signal plateaus where they are relatively insensitive to changes in $T_{1}$ or $T_{2}$ along the $X$ axis respectively and therefore show low contrast. It is in the more steeply sloping region between the two plateaus where high negative or positive contrast is produced by changes in $T_{1}$ or $T_{2}$.

The slope of the filter in Figures 4,5 is the sequence weighting for each filter. This is the first partial derivative of $\mathrm{S}_{\mathrm{T} 1}$ or $\mathrm{S}_{\mathrm{T} 2}$ respectively for small changes in $\Delta$. It is also the tangent of the angle the filter makes with the $\mathrm{X}$ axis and 
is shown in red in Figures 4,5. The $T_{1}$ and $T_{2}$-filters each provide univariate models of signal contrast and weighting in which only a single tissue property is considered at a time.

The combination of the filters (multivariate model) for $\rho_{\mathrm{m}}$

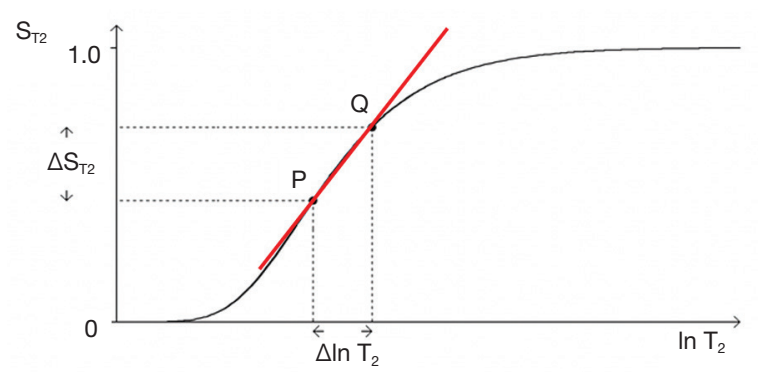

Figure 5 Plot of $\mathrm{S}_{\mathrm{T} 2}$ against $\ln \mathrm{T}_{2}$ for two tissues, $\mathrm{P}$ and $\mathrm{Q}$. The positive difference $\Delta \ln \mathrm{T}_{2}=\Delta \mathrm{T}_{2} / \mathrm{T}_{2}$ from $\mathrm{P}$ to $\mathrm{Q}$ along the $\mathrm{X}$ axis produces a positive difference in signal in signal (or contrast) $\Delta \mathrm{S}_{\mathrm{T} 2}$ from $\mathrm{P}$ to $\mathrm{Q}$ along the $\mathrm{Y}$ axis where it corresponds with a sloping region of the $\mathrm{T}_{2}$-filter. Little or no difference in signal would be produced by $\Delta \ln T_{2}$ if it was opposite either the upper or the lower flat regions of the curve. The slope of the $\mathrm{T}_{2}$-filter between $\mathrm{P}$ and $\mathrm{Q}$ which is its sequence weighting is shown in red. It is positive. (which has an exponential filter using a $\ln X$ axis), $T_{1}$ and $T_{2}$ is shown in Figure 6. For two tissues $\mathrm{P}$ and $\mathrm{Q}$, the fractional

changes in each of the tissue properties $\frac{\Delta \rho_{\mathrm{m}}}{\rho_{\mathrm{m}}}, \frac{\Delta \mathrm{T}_{1}}{\mathrm{~T}_{1}}$ and $\frac{\Delta \mathrm{T}_{2}}{\mathrm{~T}_{2}}$ shown along the $\mathrm{X}$ axes of each filter are multiplied by the slopes of their respective filters to give the contrast shown as the difference between $\mathrm{P}$ and $\mathrm{Q}$ along the $\mathrm{Y}$ axes of each filter i.e., $S_{\rho m}(A), S_{T 1}(B)$ and $S_{T 2}(C)$. The signals for $\mathrm{P}$ and $\mathrm{Q}$ along the $\mathrm{Y}$ axis of each of the three filters are then multiplied together to give the overall signals for $\mathrm{P}$ and $\mathrm{Q}$, as well as the overall signal difference, or contrast between them as shown in the lower center of Figure 6. The slopes of the curves which are their sequence weightings are shown in red in Figure 6. The slopes are positive for the $\rho_{\mathrm{m}}$ and $\mathrm{T}_{2}$-filters but negative for $\mathrm{T}_{1}$-filter.

For the IR sequence when TR is long $\left(1-\mathrm{e}^{-\mathrm{TR} / \mathrm{T} 1}\right) \approx 1$, and $\mathrm{S}_{\mathrm{T} 1}$ is largely determined by the second $\mathrm{T}_{1}$ dependent preparation period, TI, where:

$$
\mathrm{S}_{\mathrm{T} 1}=\left(1-2 \mathrm{e}^{-\mathrm{TI} T \mathrm{~T}}\right)
$$

This takes the form shown in Figure 7 for ps (A) and $\mathrm{m}$ (B)

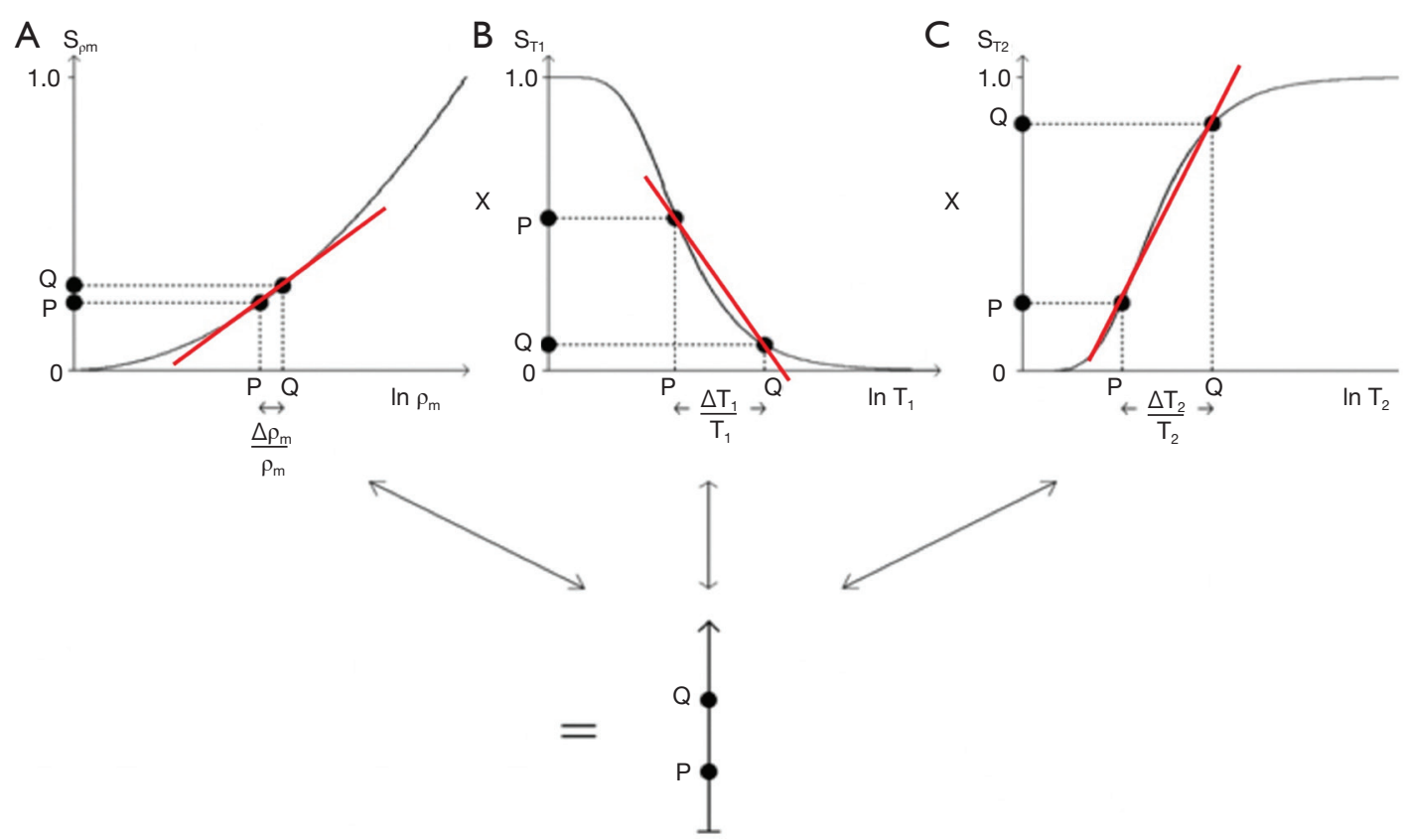

Figure 6 The SE sequence showing its $\rho_{\mathrm{m}}$-filter (A), $T_{1}$-filter (B) and $T_{2}$-filter (C). Using a $\log$ TP scale the $\rho_{\mathrm{m}}$-filter is exponential, the $\mathrm{T}_{1}$ filter is a low pass sigmoid and the $\mathrm{T}_{2}$-filter is a high pass sigmoid. The signals which $\mathrm{P}$ and $\mathrm{Q}$ produce with each filter in (A), (B) and (C) are multiplied together to give the overall contrast shown in the lower center of the diagram. The slopes of $\rho_{\mathrm{m}}, \mathrm{T}_{1}$ and $\mathrm{T}_{2}$-filters which are their respective sequence weightings are shown in red. They are positive for $\rho_{\mathrm{m}}$, negative for $T_{1}$ and positive for $T_{2}$. The contrast produced in changing each TP positively along the $\mathrm{X}$ axis from $\mathrm{P}$ to $\mathrm{Q}$ is positive for the $\rho_{\mathrm{m}}$-filter, negative for the $\mathrm{T}_{1}$-filter and positive for the $\mathrm{T}_{2}$-filter. 

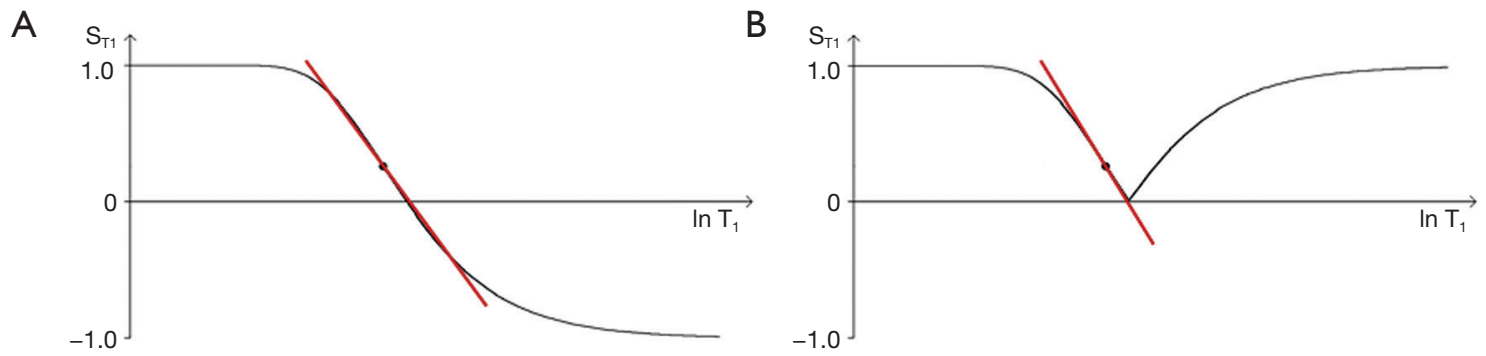

Figure 7 Long TR IR sequence filters with phase corrected (A) and magnitude (B) reconstructions. The $\mathrm{T}_{1}$-filter in (A) has high and low plateaus with a sloping region between. It has twice the range (+1 to -1), and twice the slope of the corresponding $S E T_{1}$-filter. The $T_{1}$-filter in (B) is the same as (A) up to the zero, or null point. Beyond this, it is a mirror reflection across the $\mathrm{X}$ axis of the curve shown in (A). The slopes of the curves in (A) and (B) for the maximum sequence weighting are shown in red. This is at $0.26=26 \%$ of the maximum signal. The slopes of the filters shown in (A) and (B) are opposite in polarity for $\mathrm{T}_{1}$ values greater than that at the null point.
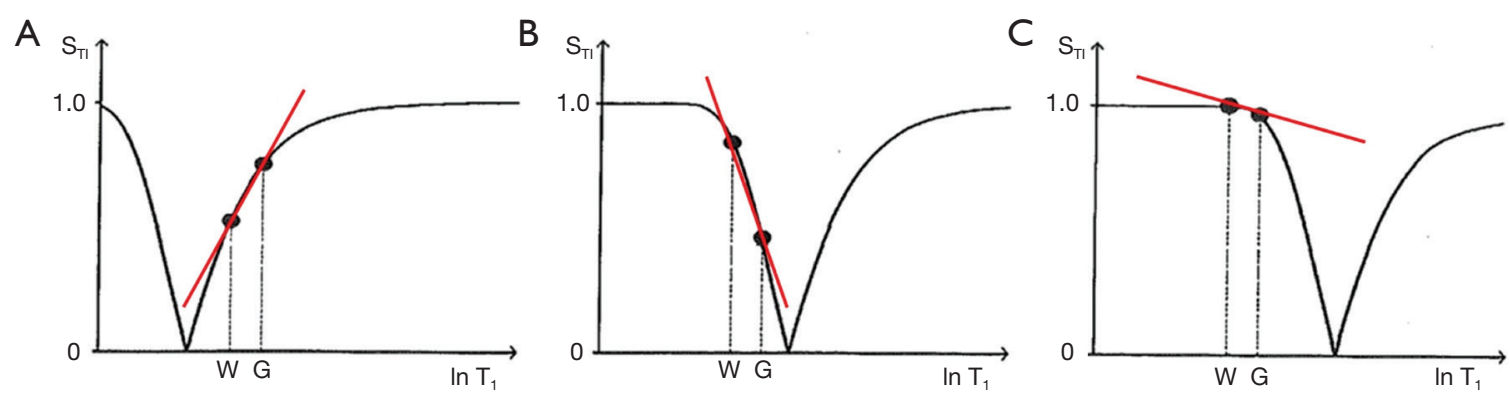

Figure 8 Plots of $S_{T 1}$ against $\ln T_{1}$ of the IR $T_{1}$-filter for short TI (A), intermediate TI (B), and long TI (C) IR sequences. The positions of white matter $(W)$ and gray matter $(G)$ of the brain along the $X$ axis are shown, and are fixed in (A), (B) and (C). In (A) (the STIR sequence), $\mathrm{G}$ is higher signal than W. In (B) (the intermediate TI sequence) the filter is shifted to the right and W is higher signal than $\mathrm{G}$, and in (C), the long TI sequence (e.g., $\mathrm{T}_{2}$-FLAIR sequence) the filter is further shifted to the right and $W$ is slightly higher signal than $G$. These are the three main classes of the long TR IR sequence. The slopes of the filters between $W$ and $G$ which are the sequence weightings of the $T_{1^{-}}$ filters are shown in red. They are highly positive in (A), highly negative in (B) and mildly negative in (C).

reconstructions. With ps reconstruction the $T_{1}$-filter has a range from -1 to $+1(\mathrm{~A})$. In the $\mathrm{m}$ form the signal range is from 0 to 1 (B). The curves show twice the slope of the $S_{\mathrm{T} 1}$ segment $\left(1-\mathrm{e}^{-\mathrm{TR} / \mathrm{Tl}}\right)$ in the SE sequence (Eqs. 1-3). The IR filter is a low pass filter with ps reconstruction (Figure $7 A$ ), and a notch filter with $\mathrm{m}$ reconstruction (Figure $7 B$ ). Its sequence weighting (the slope of the filter) at the point of maximum weighting is shown in red (Figure 7).

With increase in TI the $\mathrm{T}_{1}$-filter shifts to the right (Figures 8 ). White $(\mathrm{W})$ and Gray $(\mathrm{G})$ matter are shown in a fixed position on the $\mathrm{X}$ axes as TI is increased from (A) to (C). With short TIs as with the STIR sequence G signal is greater than that of $\mathrm{W}$ (Figure $8 A$ ), for intermediate $\mathrm{TI}_{\mathrm{s}} \mathrm{W}$ signal is greater than that of $\mathrm{G}$ (Figure $8 B$ ), and for long $\mathrm{TI}_{s}$ $\mathrm{W}$ signal is slightly greater than that of $\mathrm{G}$ (Figure $8 C$ ). This corresponds to the $T_{1}$ dependent contrast seen with the short TI (e.g., STIR), intermediate TI, and long TI (e.g., $\mathrm{T}_{2}$-FLAIR) sequences respectively. The slopes of the filters in the region between $W$ and $G$ are shown in red on each curve. The slope is highly positive in (A), highly negative in (B) and slightly negative in (C).

As explained above, sequence weighting is the first partial derivative of the filter signal equation and is the slope of each filter. Image weighting is the proportion of the image contrast due to each tissue property. For a single TP-filter (univariate model) it is $\pm 100 \%$ attributable to that tissue property (e.g., Figures $7 A, 7 B, 8 A, 8 B$ ). For a combination of tissue properties (multivariate model) (Figure 6) the relative contributions of each to the image can be calculated, including the contributions from both the change in tissue property along the $\mathrm{X}$ axis, and the sequence weighting for that tissue property (i.e., the slope of the filter). This can be 
expressed as an image weighting ratio.

In summary, TP-filters provide a graphical representation of signal, contrast, difference or change in tissue property, sequence weighting and image weighting, and assign a positive or negative sign to each of these. They can be used singly (the univariate model) (Figures $4,5,7,8$ ) or in combination (the multivariate model) (Figure 6). The combination of TP-filters is particularly helpful for showing whether contrast developed by different TP-filters in the same sequence is synergistic or opposed. TP-filters are shown in mathematical form in tables at the end of the text of this paper.

\section{Added IR (AIR), subtracted IR (S $\left.{ }^{1} I R\right)$, subtracted SIR (S IR) (same initial TI) (typically SE, GE dcs) (13)}

It is possible to reconstruct images with the same TI in both ps and $\mathrm{m}$ forms as shown in Figures $9 A, B$. These can be added as an Added IR (AIR) sequence or subtracted as a Subtracted IR ( $\left.{ }^{1} \mathrm{IR}\right)$ sequence. This section considers IR sequences with the same TI as the only step, or the initial step in combining them by addition or subtraction.

\section{$A I R\left(p s T I_{s i l l}+m T I_{s i / l}\right)$}

In Figure 9 addition of the ps (A) and $\mathrm{m}$ (B) images produces a (normalized) low pass $\mathrm{T}_{1}$-filter in (C) in which signal is seen from tissues with shorter $T_{1} s$, but is not seen from tissues or fluids with longer $T_{1}$ s. The filter has a "pass" band for the tissues which have shorter values of $T_{1}$, a "transition" band for intermediate values of $\mathrm{T}_{1}$, and a "stop" band for tissues and fluids with long values of $\mathrm{T}_{1}$. It can be used to show, for example, white matter in the brain without gray matter or CSF. Increases in $T_{1}$ in the region of the transition band result in reductions in signal.

\section{$S^{1} I R\left(p s T I_{s i / l}-m T I_{s i / l}\right)$}

In Figure 10 subtraction of the $m$ image (B) from the ps image (A) is shown. This produces a (normalized) high pass $\mathrm{T}_{1}$-filter $(\mathrm{C})$ with shorter $\mathrm{T}_{1}$ tissues zero signal, and longer $T_{1}$ tissues and fluids high signal. Increases in $T_{1}$ in the transition band result in positive change in signal.

It is also possible to choose, for example, a long $\mathrm{TI}\left(\mathrm{TI}_{1}\right)$ corresponding to a $T_{1}$ between blood and CSF (since the nulling $\mathrm{TI}=0.69 \mathrm{~T}_{1}$ ). This displaces the high pass filter to the right. With this $\mathrm{TI}_{1}$ very long $\mathrm{T}_{1}$ fluids such as CSF and urine may show signal, while blood shows zero signal.
Subtracted $S^{1} I R\left(S^{2} I R\right)\left[\left(m T I_{s / i}-p s T I_{s / i}\right)-\left(m T I_{l}-p s T I_{\nu}\right)\right]$

In Figure 11 subtraction of the SIR filter with a long $\mathrm{TI}_{1}$ (B) from the SIR filter with a short or intermediate $\mathrm{TI}_{\mathrm{s} / \mathrm{i}}$ (A) produces a (normalized) band pass $\mathrm{T}_{1}$-filter $(\mathrm{C})$ which could be used to show, for example, shorter $T_{1}$ tissues with no signal, blood with signal, and longer $\mathrm{T}_{1} \mathrm{CSF}$ with zero signal and so provide a basis for angiography or venography.

\section{SIR sequences (different TIs) (typically SE, GE dcs) (13-16)}

In this group of sequences, the only subtraction is of images with two different TIs. These images can each be constructed in ps or $\mathrm{m}$ form and be subtracted in either order (i.e., longer from shorter TIs, or shorter from longer TI, i.e., the reverse form designated by r). This provides eight options. Two of these are discussed in more detail below.

\section{$\operatorname{SIR}\left(m T I_{s}-m T I_{i}\right)$}

Subtraction of an $\mathrm{m}$ image with an intermediate $\mathrm{TI}\left(\mathrm{TI}_{\mathrm{i}}\right)$ from an $\mathrm{m}$ shorter $\mathrm{TI}\left(\mathrm{TI}_{\mathrm{s}}\right)$ image, i.e., $\left(\mathrm{mTI}_{\mathrm{s}}-\mathrm{mTI}_{\mathrm{i}}\right)$ is shown in Figure 12 with the subtraction $\mathrm{T}_{1}$-filter in blue. This has a high positive slope within its central transition band. An increase in $\mathrm{T}_{1}$ in this region produces high positive contrast. In this univariate model, signals from tissues with shorter $T_{1} s$ than that at the nulling points of $\mathrm{TI}_{\mathrm{s}}$ are negative and increase with decreasing $T_{1}$, while signals from tissues with $T_{1}$ s longer than the $T_{1}$ at $T_{i}$ are positive and decrease as $T_{1}$ increases.

If $\mathrm{p}=\mathrm{TI}_{\mathrm{i}} / \mathrm{TI}_{\mathrm{s}}$ is the ratio of the two $\mathrm{TI}_{\mathrm{s}}$, for long values of $\mathrm{TR}$ also $\mathrm{p} \approx \mathrm{TI}_{\mathrm{i}} / \mathrm{TI}_{\mathrm{s}}$ where $\mathrm{T} 1_{\mathrm{i}}$ and $\mathrm{T} 1_{\mathrm{s}}$ are the values of $\mathrm{T}_{1}$ at the respective nulling points. Using Eq. 4 the normalized signal difference SD between the high and low peaks of the filter at the $\mathrm{T} 1_{\mathrm{s}}$ of the short and long $\mathrm{TI}$ nulling points is approximately given by:

$$
S_{D}=\left(e^{-\frac{1}{p}}-e^{-p}\right)
$$

As $\mathrm{p}$ increases from 1 the amplitude of the signal difference increases from zero up to a maximum of 1 (Figure 13).

The slope of the curve between the two null points is approximately given by:

$$
\frac{\mathrm{dS}_{\mathrm{D}}}{\mathrm{dp}}=\frac{\mathrm{e}^{-\frac{1}{\mathrm{p}}}}{\mathrm{p}^{2}}+\mathrm{e}^{-\mathrm{p}}
$$



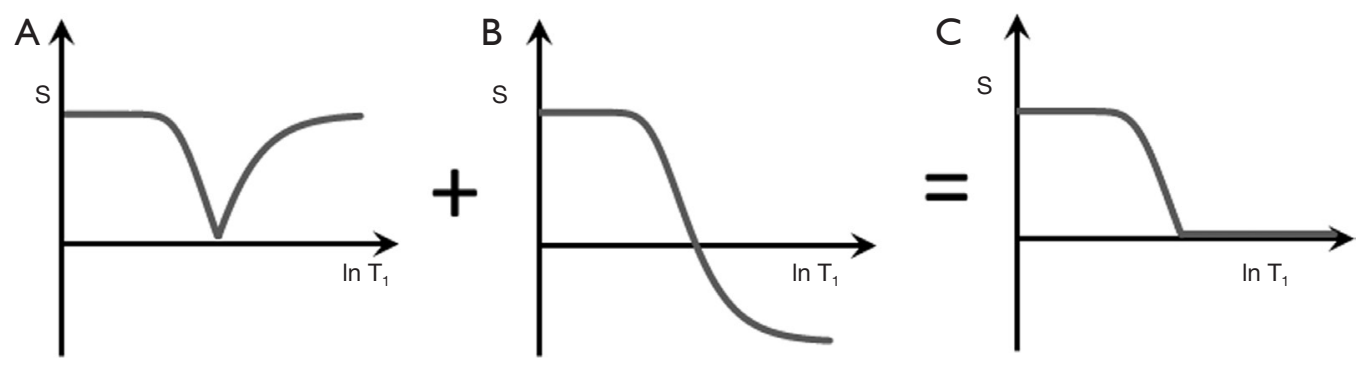

Figure $9 \mathrm{~T}_{1}$-filter for Added IR (AIR) ( $\left.\mathrm{ps}^{T} \mathrm{TI}_{\mathrm{s} / \mathrm{i} / 1}+\mathrm{mTI}_{\mathrm{s} / \mathrm{i} / \mathrm{l}}\right)$ sequence. The ps sequence at any short, intermediate or long $\mathrm{TI}(\mathrm{TI} / \mathrm{s} / \mathrm{l})(\mathrm{A})$ is added to the $m$ reconstructed version of the same sequence with the same TI (B) to give a low pass $T_{1}$-filter in (C). The $S$ values are normalized in this filter. In (C) signal is seen from shorter values of $T_{1}$, there is then a transition band as $T_{1}$ increases, and no signal is seen for longer values of $T_{1}$.
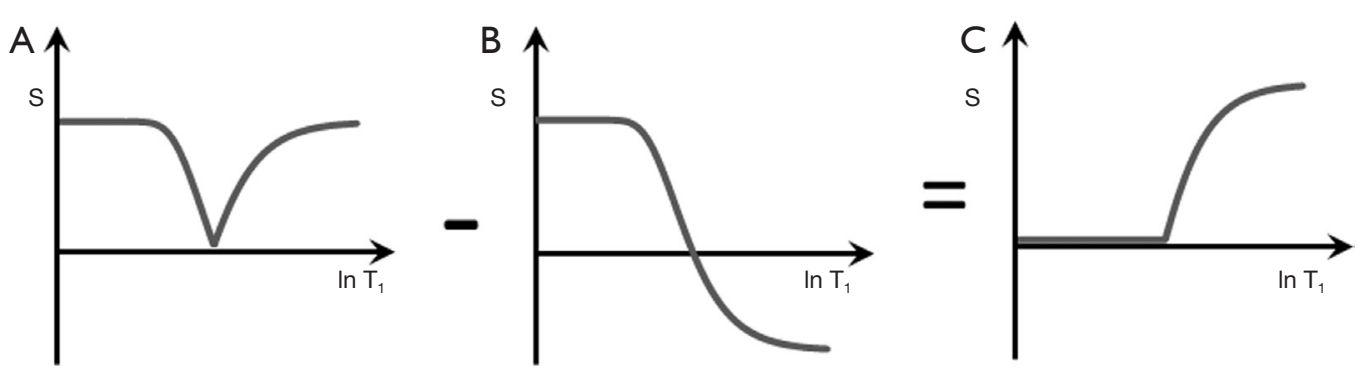

Figure $10 \mathrm{~T}_{1}$-filter for subtracted $\mathrm{IR}\left(\mathrm{S}^{1} \mathrm{IR}\right)\left(\mathrm{mTI}_{\mathrm{s} / \mathrm{i} / \mathrm{p}}\right.$ - ps $\left.\mathrm{TI}_{\mathrm{s} / \mathrm{i} / \mathrm{f}}\right)$ sequence. The $\mathrm{m}$ sequence (A) at any short, intermediate or long $\mathrm{TI}$ (TIs, $\left.\mathrm{TI}_{\mathrm{i}}, \mathrm{TI}_{1}\right)$ has subtracted from it the corresponding ps sequence with the same TI (B). This results in a (normalized) high pass $\mathrm{T}_{1}$-filter $(\mathrm{C})$. With this filter short $T_{1}$ values are zero. There is a transition band as $T_{1}$ increases and higher signals are seen as $T_{1}$ increases further.

This slope is maximum at $\mathrm{p}=1$ and decreases to zero as $\mathrm{p}$ increases.

With this sequence within the transition band there is the potential for nearly doubling again the already double $\mathrm{T}_{1}$ sequence weighting of the IR sequence relative to a $\mathrm{SE}$ sequence $T_{1}$-filter. This is achieved by subtracting from the rapidly rising curve $\left(\mathrm{TI}_{\mathrm{s}}\right)$ the rapidly decreasing curve $\left(\mathrm{TI}_{\mathrm{i}}\right)$ so that the latter also makes a positive contribution to contrast when $\mathrm{T}_{1}$ increases.

For the short $\mathrm{TI}_{\mathrm{s}} \mathrm{IR}$ acquisition alone (e.g., with white matter nulling) the $\rho_{\mathrm{m}}$ and $T_{2}$ sequence weightings are positive and synergistic with the $T_{1}$ sequence weighting in the transition band for an increase in $\rho_{\mathrm{m}}, \mathrm{T}_{1}$ and $\mathrm{T}_{2}$. For the intermediate $\mathrm{TI}_{\mathrm{i}} \mathrm{IR}$ acquisition alone (e.g., for gray matter nulling) the $T_{1}$ sequence weighting in the transition band is negative while the $T_{2}$ sequence weighting is positive. The latter can be minimised by using a short or ultrashort TE. It can be made negative and become synergistic with the $T_{1}$ sequence weighting by using echo subtraction (ES) (see later) to reverse the $T_{2}$ sequence weighting and make it negative. This increases contrast when the $T_{i} I R$ sequence is subtracted from the $\mathrm{TI}_{s} \mathrm{IR}$ sequence as with the subtracted IR echo subtraction (SIRES) sequence.

Relative to the $T_{2}$-FLAIR sequence, the $T_{1}$ contrast in the transition band with the SIR sequence is: (i) synergistic with that produced by $\rho_{\mathrm{m}}$ and $T_{2}$ for an increase in all three tissue properties, not opposed to them, and (ii) larger in size. Relative to the $T_{2}$-weighted $\mathrm{SE}$ sequence the $T_{1}$ contrast of the SIR sequence is high, not zero or near zero, and is synergistic with that of $\rho_{\mathrm{m}}$ and $\mathrm{T}_{1}$ for increases in all three tissue properties. In addition, the signal from CSF is much lower with the SIR sequence compared to the $T_{2}$ weighted SE sequence.

\section{$r S I R\left(m T I_{i}-m T I_{S}\right)$ (reverse subtraction IR)}

The $T_{1}$-filters for forward i.e., the default (A) and reverse (B) subtraction $\left(\mathrm{mTI}_{\mathrm{i}}-\mathrm{psTI} \mathrm{I}_{\mathrm{s}}\right)$ are shown in Figure 14. In Figure $14 B$, as $T_{1}$ increases, there is positive increasing signal, then a highly negative sloping region in the transition band between the $\mathrm{T}_{1} \mathrm{~s}$ corresponding to the null points for $\mathrm{TI}_{s}$ and $\mathrm{TI}_{\mathrm{i}}$ followed by a negative signal 

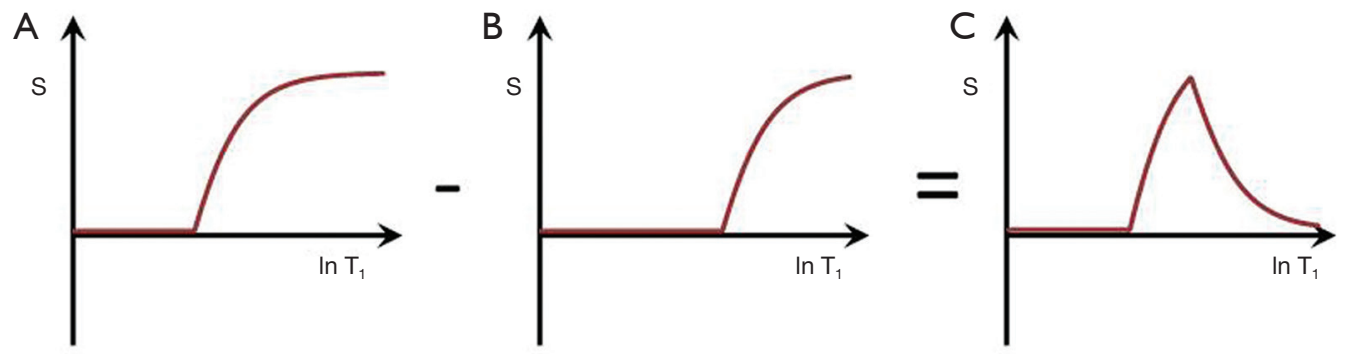

Figure 11 Subtracted $S^{1} I R\left[S^{2} I R\left(\mathrm{mTI}_{\mathrm{s} / \mathrm{i}}-\mathrm{ps}_{\mathrm{TI} / \mathrm{i}}\right)-\left(\mathrm{mTI}_{1}-\mathrm{ps} \mathrm{TI}_{1}\right)\right]$ sequence. The high pass filter in $(\mathrm{B})$ with a long $\mathrm{TI}_{1}$ produced by subtracting a ps image from an $\mathrm{m}$ image, is subtracted from the high pass filter in $(\mathrm{A})$ which has a short or intermediate $\mathrm{TI}\left(\mathrm{TI}_{\mathrm{s}}\right.$ or $\left.\mathrm{TI}_{\mathrm{i}}\right)$. This gives the band pass filter shown in (C). With longer values of TI this could be used to selectively image blood.

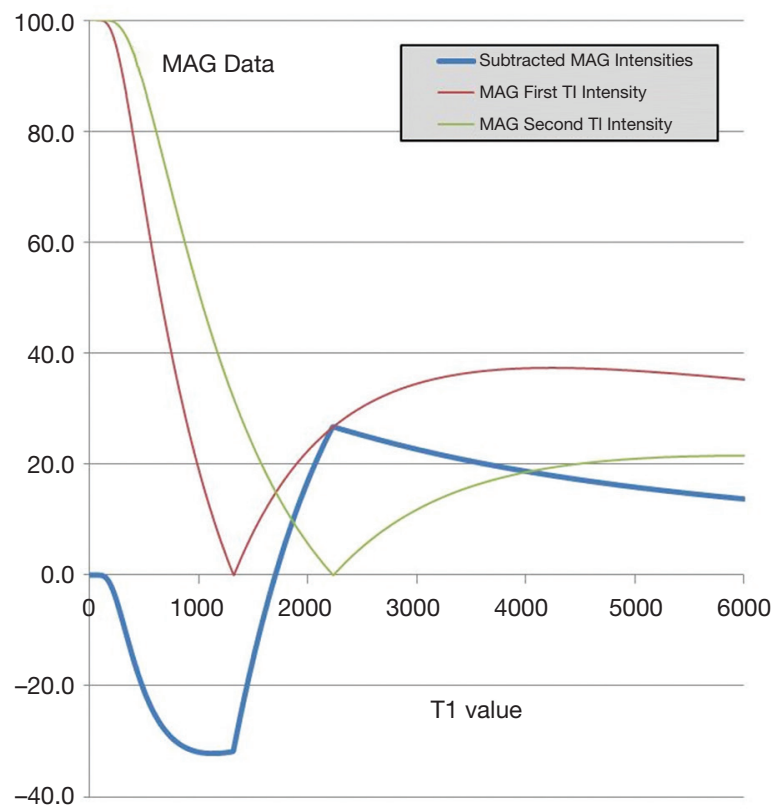

Figure $12 \mathrm{~T}_{1}$-filter for SIR $\left(\mathrm{mTI}_{\mathrm{s}}-\mathrm{mTI}_{\mathrm{i}}\right)$ filter. An $\mathrm{m}$ reconstructed intermediate $\mathrm{TI}\left(\mathrm{TI}_{\mathrm{i}}\right)$ (green image is subtracted from a $\mathrm{m}$ reconstructed short TI (TIs) image (red). The difference image (blue) shows that for $T_{1} s$ in the range between the null points of the two TIs there is a very high positive slope or sequence weighting. The slope of the subtracted curve in this range is greater than that of either of the two original images. This leads to high positive contrast for increases in $T_{1}$ in the transition band.

for longer $T_{1}$ tissues and fluids. This reverse subtraction $\left(\mathrm{mTI}_{\mathrm{i}}-\mathrm{mTI}_{\mathrm{s}}\right)$ can be used to provide low signal for gray matter, with very high negative $T_{1}$ sequence weighting leading to high positive signal when there is a decrease in $\mathrm{T}_{1}$ in gray matter (if, for example, it is the tissue nulled at $\mathrm{TI}_{\mathrm{i}}$ ). This may be seen with iron deposition and contrast agents such as Gd-DTPA. The sequence does not show contrast enhancement (i.e., increase in signal) for tissues with $\mathrm{T}_{1} \mathrm{~s}$ corresponding to $\mathrm{TI}_{\mathrm{s}}$ or shorter, or for tissues with $T_{1}$ s longer than those corresponding to $\mathrm{TI}_{\mathrm{i}}$ with decreases in $T_{1}$.

GE/EPI acquisitions can be used to increase the sensitivity of the sequence to susceptibility effects from iron or Gd-DTPA.

The values of $\mathrm{TI}_{\mathrm{s}}$ and $\mathrm{TI}_{\mathrm{i}}$ may need to be shortened and lengthened respectively to widen the range of values of $T_{1}$ which show positive contrast with decreases in $T_{1}$, at the expense of reducing the slope of the filter in the transition band, and so producing less contrast.

\section{Multiplied IR (MIR), DIR, TIR, QIR, MP-RAGE, MSIR and FLAIR ${ }^{2}$ Sequences (typically SE, GE dcs)}

Multiplied IR (MIR) sequences multiply two or more IR sequences or images together. Examples are discussed below.

\section{The DIR sequence in the brain}

Figure 15 shows multiplication of the $\mathrm{T}_{1}$-filter of a long TI IR sequence nulling CSF (A) by a short TI sequence nulling white matter (B) to give the $\mathrm{T}_{1}$-filter shown in $(\mathrm{C})$ which is of double notch form. This is a DIR sequence used to selectively null white matter and CSF and so exclusively show gray matter (5).

\section{The DIR sequence in the body}

In the body (apart from the heart) it is usual to null fat and fluid with the DIR sequence but otherwise it is similar to that shown in Figure $15(4,17)$. 


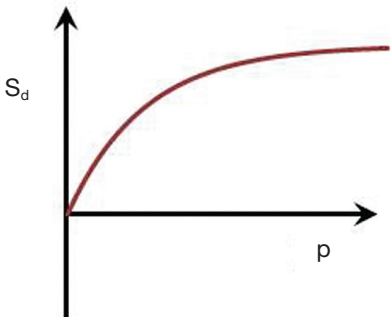

Figure 13 Plot of signal difference $\left(\mathrm{S}_{\mathrm{D}}\right)$ between the high and low peaks on the SIR image shown in Figure 13 (blue curve) against $\mathrm{p}$ (ratio of $\mathrm{TI}_{\mathrm{i}} / \mathrm{TI}_{\mathrm{s}}$, i.e., the value of $\mathrm{TI}$ to null the intermediate $\mathrm{T}_{1}$ tissue to the value of TI to null the short $T_{1}$ tissue). Signal increases to 1 as $p$ increases. The slope of the curve is highest at $\mathrm{p}=1$ and decreases as $\mathrm{p}$ increases. As $\mathrm{p}$ increases there is more signal, but less contrast.

\section{The DIR sequence in the beart}

The term DIR is used in cardiology to describe an initial non-selective inversion pulse followed soon after by a sliceselective inversion pulse through the heart (18). This inverts then reinverts the heart longitudinal magnetization within the slice to leave it relatively unaffected. The longitudinal magnetization of the blood outside the cardiac slice remains inverted. When this outside blood flows into the slice, a value of TI can be chosen to selectively null its signal and so, after a $90^{\circ}$ pulse is applied, provide high contrast between zero signal blood and high signal myocardium around it. This is described as "black blood" imaging. The reinverted blood originally in the cardiac slice flows elsewhere.

\section{The triple IR (TIR) sequence in the heart}

The term TIR is used to describe the cardiac version of DIR as outlined above with an additional slice-selective inversion pulse before the $90^{\circ}$ pulse to null signal from fat and potentially provide synergistic $T_{1}$ and $T_{2}$ contrast (19). The additional inversion pulse causes re-inversion of the inflowing blood and requires adjustment of the TI to null it.

\section{The TIR sequence for angiography}

The term TIR is also used in angiography to describe the use of two non-slice-selective inversion pulses to null tissue signals over a wide range of $T_{1}$ values and selectively show them with a low or zero background $(20,21)$, together with a slice selective inversion pulse to reinvert in-flowing blood.
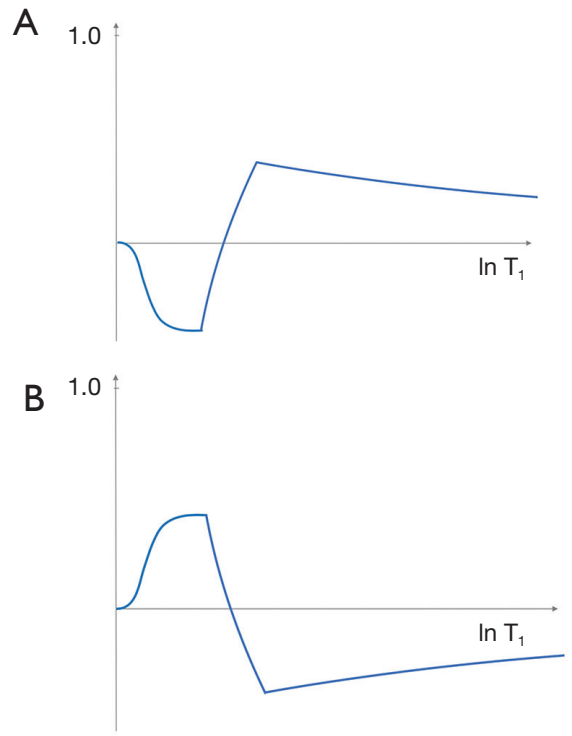

Figure $14 \mathrm{~T}_{1}$-filter for forward $\mathrm{SIR}\left(\mathrm{mTI}_{\mathrm{s}}-\mathrm{mTI}_{\mathrm{i}}\right)(\mathrm{A})$ and reversed rSIR $\left(\mathrm{mTI}_{\mathrm{i}}-\mathrm{mTI}_{\mathrm{s}}\right)(\mathrm{B})$. Between the null points for $\mathrm{TI}_{\mathrm{s}}$ and $\mathrm{TI}_{\mathrm{i}}$ the $T_{1}$-filter in (A) has a marked positive slope while the $T_{1}$ filter in (B) has a marked negative slope. In (B) reductions in $T_{1}$ from the intermediate $\mathrm{TI}_{\mathrm{i}}$ null point produce marked increases in signal. This occurs with $T_{1}$ shortening due to Gd-DTPA or iron deposition.

This is followed by a $90^{\circ}$ pulse.

\section{The quadruple IR (QIR) sequence for angiography}

The term QIR is used both to describe the use of two DIR pulses to provide a very wide range of tissue signal suppression and so allow detection of inflowing blood (22), and to describe a form of angiography in which a nonselective inversion pulse is followed by a selective slice selection of reinverted blood in the vessel of interest, a slice selective pulse is used to null venous blood, and a short TI inversion is used to null fat (23).

\section{Magnetization prepared rapid acquisition gradient echo (MP-RAGE), MP-2RAGE and MPnRAGE sequences}

(i) MP-RAGE is a 3D GE based sequence which usually uses an intermediate TI to provide high gray white matter contrast in the brain with white matter having the higher signal (24).

(ii) The MP-2RAGE sequence includes an additional 


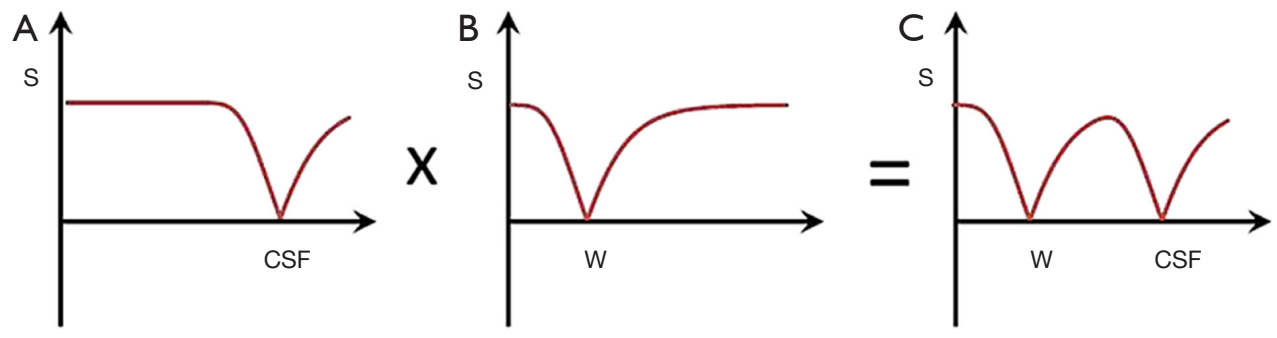

Figure 15 The DIR sequence for imaging the brain. (A) is the $T_{1}$-filter nulling CSF, (B) is the $\mathrm{T}_{1}$-filter nulling white matter (W) and (C) is (A) multiplied by (B) in which both $\mathrm{W}$ and CSF are nulled. The sequence weighting is the slope of the $\mathrm{T}_{1}$-filter in $(\mathrm{C})$. It is zero, negative and positive for different values of $T_{1}$. CSF, cerebrospinal fluid.

interleaved inversion pulse so that data with two TIs are acquired. These are multiplied together and normalized. The MP-2RAGE sequence is usually optimized to provide high gray white matter contrast with white matter having the higher signal $(25,26)$.

(iii) Two variants of the MP-2RAGE sequence are FLAWS1 and FLAWS2 (Fluid And Water Suppressed versions 1 and 2) which have features of the MPRAGE sequence as well as features of the DIR sequence in which white matter signal is suppressed and fluid signal is suppressed (27).

(iv) Another variant of MP-2RAGE is one in which Null Point Imaging (NPI) is employed. This nulls tissue at a TI between that of gray and white matter and aims to have their signals of equal magnitude. It may use $\mathrm{m}$ or ps reconstruction $(28,29)$.

(v) MPnRAGE. The MPnRAGE sequence uses radial $\mathrm{k}$-space mapping with a sliding window acquisition and progressively steps through a range of TIs (30). It can produce a large number of IR images and is similar to the DESIRE-UTE (Double Echo Sliding IRE-UTE) sequences (31) but uses a single RAGE data acquisition rather than the double echo acquisition of the DESIRE-UTE version. The MPnRAGE sequence as originally proposed is a fitted sequence designed to measure T1 not a MIR sequence, however the acquired images can also be multiplied, added or subtracted.

\section{The MSIR sequence}

With this sequence an additional initial inversion pulse (Figure 16A) is used to reduce the long $\mathrm{T}_{1}$ fluid signal of the SIR sequence. This has the effect of multiplying Figure 12 shown in Figure $16 B$ by an extra initial segment to give
Figure 16C. It can reduce the signal from long TI fluids such as CSF to zero. In addition, the $\mathrm{TE}$ of the $\mathrm{TI}_{1}$ sequences is usually shortened to reduce opposed $T_{2}$ contrast. This sequence can also be regarded as the subtraction of two DIR sequences with the same initial long TI $\left(\mathrm{TI}_{1}\right)$ value but shorter and longer second $\mathrm{TI}$ values i.e., $\mathrm{TI}_{\mathrm{s}}$ and $\mathrm{TI}_{\mathrm{i}}$ for the two DIR sequences.

\section{$F_{L A I R^{2}}$}

It is also possible to multiply a $T_{2}$-FLAIR sequence by a $\mathrm{T}_{2}$-weighted $\mathrm{SE}$ sequence to give the $\mathrm{FLAIR}^{2}$ sequence (32). This retains the low CSF signal of the $T_{2}-$ FLAIR sequence and increases its $\mathrm{T}_{2}$-weighting. It is an example of the multiplication of an IR sequence by another type of sequence.

\section{Echo subtraction (ES)}

Another way of reducing the longer $T_{2}$ signal is subtraction of an image with a longer TE (e.g., short TEs, intermediate $\mathrm{TE}_{\mathrm{i}}$ and long $\mathrm{TE}_{\mathrm{l}}$ ) from one with a shorter $\mathrm{TE}$ (e.g., $\mathrm{TE}_{\mathrm{u}}$ ) (Figures 17 I-III). With the first echo at $\mathrm{TE}_{\mathrm{u}}$ and the second echo at TEs this produces a relatively narrow band pass $\mathrm{T}_{2}$ filter (Figure $17 \mathrm{I}$, top row).

With a longer intermediate TEi, as shown in the second row (II), ES produces a broader band pass $\mathrm{T}_{2}$-filter. With this filter for $\mathrm{T}_{2} \mathrm{~s}$ in the intermediate range, signal decreases as $\mathrm{T}_{2}$ increases (Figure $17 \mathrm{II}$, middle row). This reverses the contrast behavior of a conventional SE sequence. For a long $\mathrm{TE}_{1}$ as the second echo, the band pass filter is broader again and the effect is primarily suppression of very long $T_{2}$ signals (Figure 17 III, lower row) $(33,34)$.

Figure 18 shows the use of an intermediate $\mathrm{TE}_{\mathrm{i}}$ subtraction from $\mathrm{TE}_{\mathrm{u}}$ in the multivariate form with the 

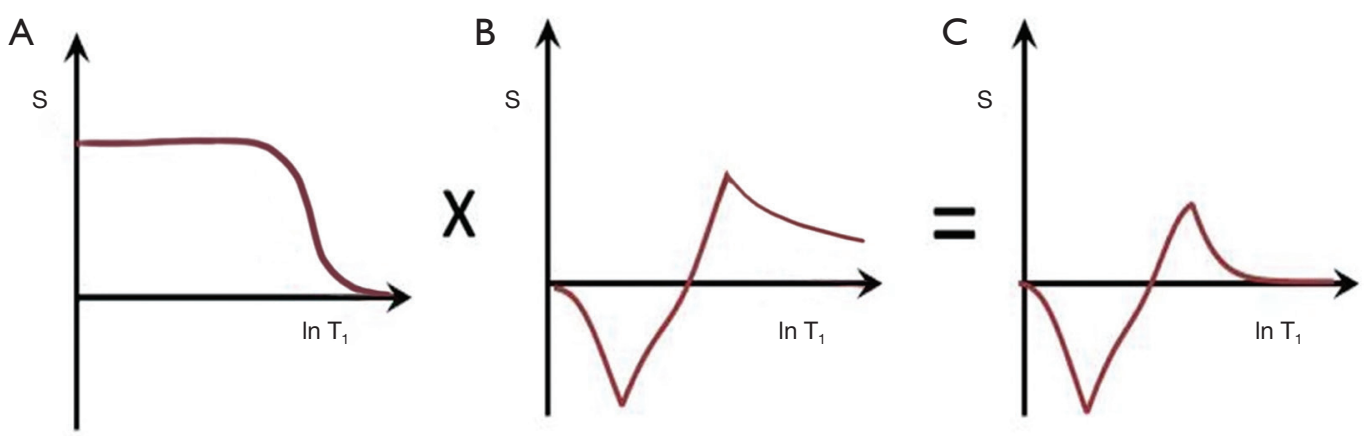

Figure 16 The MSIR sequence. This sequence multiplies a long $\mathrm{T}_{1}$-filter (A) by the SIR sequence $\mathrm{T}_{1}$-filter shown in Figure 13 (blue curve) to give the MSIR $\mathrm{T}_{1}$-filter $(\mathrm{C})$. This is similar to (B) but the signal at high values of $\mathrm{T}_{1}$ is lower.

SIRES sequence. In the top row (I) for a positive increase in each of $\rho_{\mathrm{m}}, T_{1}$ and $T_{2}$ from $\mathrm{P}$ to $\mathrm{Q}$, the positive sequence weighting of each filter results in high positive contrast from $\mathrm{P}$ to $\mathrm{Q}$.

In the middle row (II), the $\rho_{\mathrm{m}}$ sequence weighting is positive, the $T_{1}$-filter sequence weighting with the longer $\mathrm{TI}_{\mathrm{i}}$ filter is negative, and so is the sequence weighting for the ES filter $\mathrm{S}_{\mathrm{TE} 2}-\mathrm{S}_{\mathrm{TE} 1}$. For the same increase from $\mathrm{P}$ to $\mathrm{Q}$ of each of $\rho_{\mathrm{m}}, T_{1}$ and $T_{2}$ this results in positive contrast for $\rho_{\mathrm{m}}$ and negative contrast for $\mathrm{T}_{1}$ and $\mathrm{T}_{2}$. The product (right side) shows negative contrast from $\mathrm{P}$ to $\mathrm{Q}$.

In the bottom row (III), normalized subtraction of the middle row signals for $\mathrm{P}$ and $\mathrm{Q}$ (i.e., the product) from the upper row signals from $\mathrm{P}$ and $\mathrm{Q}$ (i.e., the product) is shown. This produces even higher positive contrast from $\mathrm{P}$ to $\mathrm{Q}$. The final subtraction image produces little or no contrast for $\rho_{\mathrm{m}}$ but synergistic high contrast for concurrent increases in $T_{1}$ and $T_{2}$.

\section{Diffusion (D*) and diffusion subtraction (DS) (typically SE, EPI des)}

Diffusion-Weighted whole-body Imaging with background Body Signal suppression (DWIBS) (35) usually employs a STIR sequence for fat signal suppression. This gives more consistent performance in the presence of variations in susceptibility in the body than spectral fat saturation. In addition it produces synergistic positive contrast with concurrent increases in $\rho_{\mathrm{m}}, \mathrm{T}_{1}$ and $\mathrm{T}_{2}$ providing that the $\mathrm{D}^{*}$ of the lesion is reduced. This is so for many tumors, acute cerebral infarction and other conditions. When $\mathrm{D}^{*}$ is reduced the synergistic $T_{1}$ contrast produced by the STIR sequence can be increased by increasing the TI and using chemical shift based fat saturation.
In addition to an increase in $T_{1}$ and $T_{2}$ lesions often show an increase in $\mathrm{D}^{*}$. The $\mathrm{T}_{2}$ and $\mathrm{D}^{*}$ contrast is then opposed resulting in a net loss in contrast from these two tissue properties. This can be reversed by using diffusion subtraction [DS i.e., D without sensitization (Do) minus $\mathrm{D}$ with sensitization (Dw)] (Figure 19). It makes the $\rho_{\mathrm{m}}, \mathrm{T}_{1}$ and $T_{2}$ and $D^{*}$ contrast positive and synergistic when using a TIs sequence. DS also provides fat suppression since fat is largely unaffected by diffusion weighting, but signal may be seen from fluids since the signal from fluid is not reduced by diffusion effects in the unsensitised Do image, but reduced in the sensitized Dw image. An additional long TI inversion pulse may be used to suppress long $T_{1}$ signal in the unsensitizied image so that when unsensitized and sensitized images are subtracted, the fluid signal is low or zero. If fluid does not feature in the diffusion subtracted images fluid suppression may not be required. The fluid signal on the unsensitized Do image can also be suppressed by use of long TE ES.

For an increase in $\rho_{\mathrm{m}}, \mathrm{T}_{1}$ and $\mathrm{T}_{2}$ with a decrease in $\mathrm{D}^{*}$ subtraction of the $\mathrm{TI}_{\mathrm{i}} \mathrm{IR}$ sequence as a whole from the TIs IR sequence as a whole gives synergistic positive contrast for $T_{1}, T_{2}$ and $D^{*}$ with no contrast sensitivity for $\rho_{\mathrm{m}}$ from $\mathrm{P}$ to Q. This can be seen in Figure 20 in multivariate form where a TIs sequence with synergistic positive $T_{1}, T_{2}$ and $\mathrm{D}^{*}$ contrast is shown in the top row (I). An intermediate TIi sequence using ES and DS produces synergistic negative $\mathrm{T}_{1}, \mathrm{~T}_{2}$ and $\mathrm{D}^{*}$ contrast from $\mathrm{P}$ to $\mathrm{Q}$ in the middle row (II). In the bottom row (III), normalized subtraction of the middle row resultant product signals from $\mathrm{P}$ and $\mathrm{Q}$ from the top row resultant signals leads to a further increase in the positive contrast from $\mathrm{P}$ to $\mathrm{Q}$. The normal $\mathrm{D}^{*}$ anisotropy in white matter with $\mathrm{D}^{*}$ higher for fibers parallel to the sensitizing gradient than for $\mathrm{D}^{*}$ perpendicular to the 

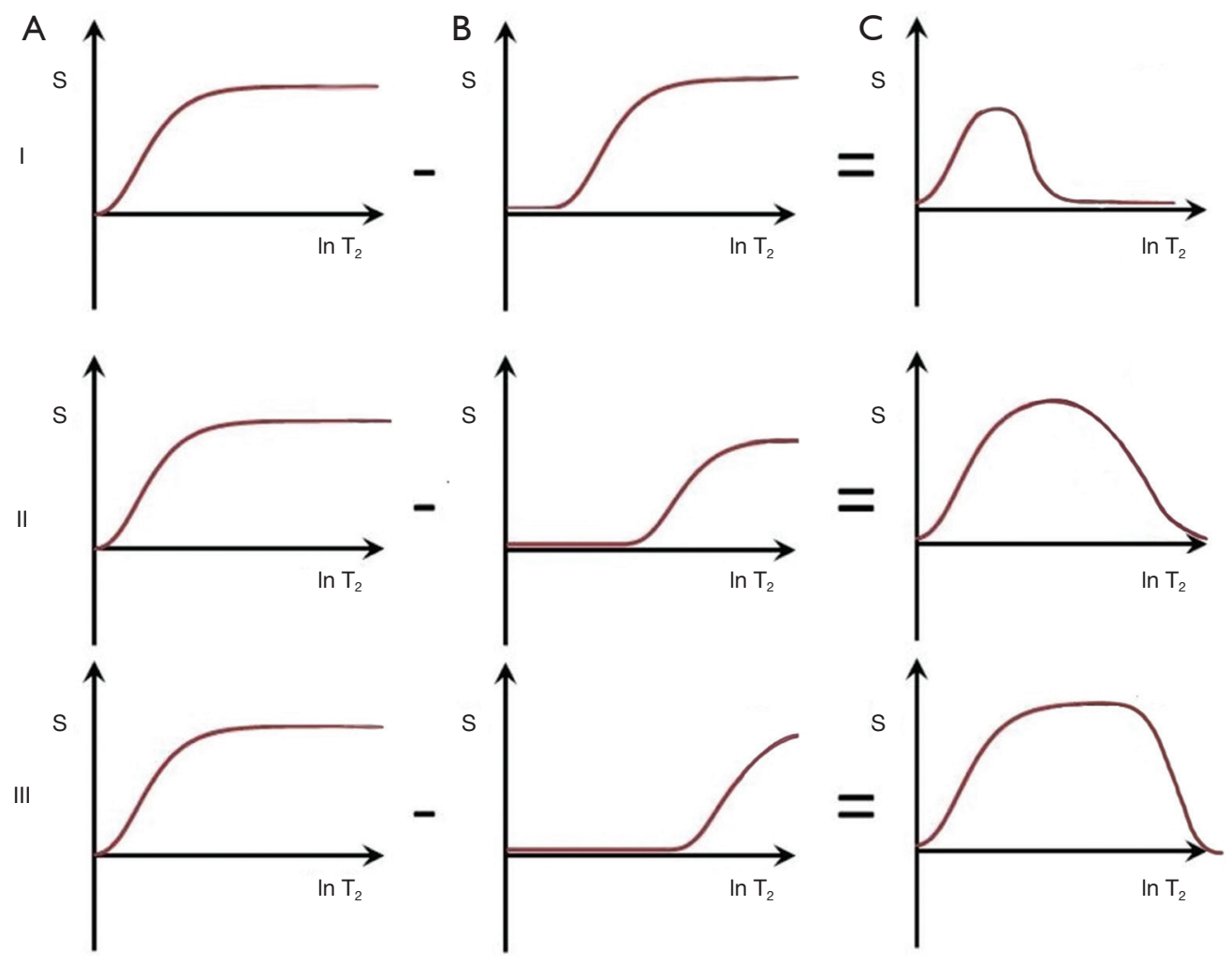

Figure 17 Echo subtraction (ES) $\mathrm{T}_{2}$-filter. Subtraction from an ultrashort TE image $\left(\mathrm{TE}_{\mathrm{u}}\right)$ of respectively a short $\mathrm{TE}$ image $\left(\mathrm{TE}_{\mathrm{s}}\right)$ is shown in the upper row (I), subtraction of an intermediate $\mathrm{TE}_{\mathrm{i}}$ in the middle row (II), and subtraction of a long $\mathrm{TE}_{\mathrm{l}}$ in the lower row (III) (C). This results in a narrow band pass filter (top row) (C), a broader band pass filter (middle row, C) and a broader still filter (lower row, $\mathrm{C}$ ). In the top row a small range of $\mathrm{T}_{2} \mathrm{~s}$ show high signal with adjacent positive and negative sequence weighting. In the middle row, for medium $\mathrm{T}_{2}$ values the slope of the curve is negative and this is the reverse of the usual SE sequence weighting. In the bottom row (C) the signal from very long $T_{2}$ fluids is suppressed.

gradient may cause confusion with increased $\mathrm{D}^{*}$ in white matter due to disease.

Figure 21 using a short $\mathrm{TI}_{\mathrm{s}}$ and DS (row I column D) shows synergistic positive contrast for increases in $\rho_{\mathrm{m}}, \mathrm{T} 1$, $\mathrm{T}_{2}$ and $\mathrm{D}^{*}$ from $\mathrm{P}$ to $\mathrm{Q}$. In row II ES (column C) is used to reverse the contrast produced by the $T_{2}$-filter. It is not possible to do this and retain the sequence weighting of the $\mathrm{D}^{*}$ filter. This would require a heavily $\mathrm{D}^{*}$ weighted sequence with a short or ultrashort TE which is not attainable with a PGSE sequence on clinical systems. The $\mathrm{T}_{1^{-}}$and $\mathrm{T}_{2}$-filters produce negative contrast. The $\mathrm{D}^{*}$ filter in row II (shown in dashed lines) is not included. Row III shows subtraction of the row II product from the row I product and this produces higher positive contrast from $\mathrm{P}$ to $\mathrm{Q}$ than row I alone.

Use of a short TIs and DS with a gradient echo diffusion sequence as the SIR Diffusion Gradient Echo Subtraction sequence (SIRDGES) gives positive contrast from $\mathrm{P}$ to $\mathrm{Q}$ for concurrent increases in $\rho_{\mathrm{m}}, \mathrm{T}_{1}, \mathrm{~T}_{2}$ and $\mathrm{D}^{*}$.

For a decrease in $T_{1}$ and $T_{2}{ }^{*}$ with an increase in $\mathrm{D}^{*}$ as with iron deposition use of a short TIs and ES as the SIRDGES sequence results in positive synergistic contrast for $\mathrm{T}_{1}, \mathrm{~T}_{2}$ and $\mathrm{D}^{*}$.

In tumors of the prostate in the peripheral and transitional zones typically each of $T_{1}, T_{2}$ and $D^{*}$ is decreased relative to normal tissue. In this case synergistic positive and negative contrast can be achieved using ES and DS but ES with unaffected $D^{*}$ contrast in one of rows I or II is not possible.

When changes in $T_{2}$ and $D^{*}$ are opposed the $\mathrm{D}^{*}$ filter can be included in both rows I and II but when they are both either increased or decreased this is not possible with the PGSE sequence and only one of the two rows can have a synergistic $D^{*}$ filter. 

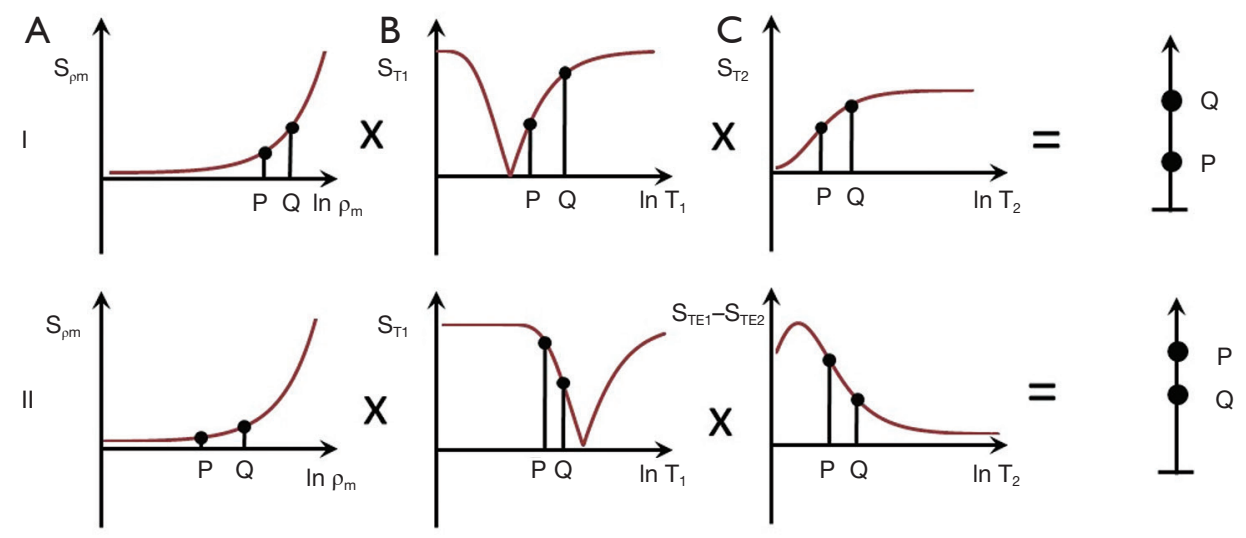

III

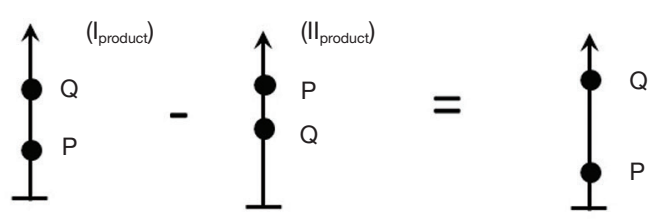

Figure 18 SIR Echo Subtraction (SIRES) sequence (multivariate model). The $\rho_{\mathrm{m}}, \mathrm{T}_{1}$ and $\mathrm{T}_{2}$-filters for a short TIs sequence are shown in the top row (I). For an increase in $\rho_{\mathrm{m}}, \mathrm{T}_{1}$ and $\mathrm{T}_{2}$ from $\mathrm{P}$ to $\mathrm{Q}$ this results in an increase in signal from $\mathrm{P}$ to $\mathrm{Q}$ in each filter, and multiplication of each of the filters shows high positive contrast from $\mathrm{P}$ to $\mathrm{Q}$ (right hand side). In the middle row (II) an intermediate TIi is used and the $\mathrm{T}_{1}$-filter sequence weighting from $\mathrm{P}$ to $\mathrm{Q}$ is negative. The $\mathrm{ES} \mathrm{T}_{2}$-filter $\left(\mathrm{S}_{\mathrm{TE} 1}-\mathrm{S}_{\mathrm{TE} 2}\right)$ is also negative from $\mathrm{P}$ to $\mathrm{Q}$. The multiplied result shows negative contrast from $\mathrm{P}$ to $\mathrm{Q}$ (right hand side). The bottom row (III) shows normalized subtraction of the signals from $\mathrm{P}$ and $\mathrm{Q}$ in the middle row (II product) from the corresponding signals in the first row (I product). This creates even higher positive contrast from $\mathrm{P}$ to $\mathrm{Q}$ than from the top row alone.

\section{Phase imaging (typically UTE, ZTE and GE, SE des)}

MR images are usually displayed in magnitude form, but phase maps can also be created. Phase differences arise from $B_{O}$ inhomogeneity, susceptibility differences, chemical shift and contrast agent effects and are typically seen with GE sequences. Phase differences can also be created by application of pulsed gradients with PGSE sequences when there is fluid flow with the phase difference proportional to the fluid velocity. Phase difference maps are created by obtaining an initial phase image at $\mathrm{TE}_{1}$ with signal $\theta_{\mathrm{TE} 1}$ and subtracting from it a second image with signal $\theta_{\mathrm{TE2}}$ obtained at a second $\mathrm{TE}_{2}$.

Inversion pulses can also produce phase differences. They are a consequence of the fact that following an inversion pulse and recovery for time TI, different tissues may show either positive or negative longitudinal magnetization. When the magnetizations of these tissues are rotated through $90^{\circ}$ into the transverse plane the transverse magnetizations of tissues which had positive longitudinal magnetization become $180^{\circ}$ out of phase with those of tissues which had negative longitudinal magnetizations. This is seen with SE dcs as well as GE dcs, and is present immediately after rotation by the $90^{\circ}$ pulse.

This phase difference differs from fat-water phase changes due to chemical shift. The signals from fat and water (or water containing tissue) are in phase immediately after excitation by the $90^{\circ}$ pulse and evolve to become out of phase as a function of their chemical shift and the strength of $\mathrm{B}_{0}$. With increasing TE the signals come back into phase and then move out of phase again. This does not occur with the out of phase transverse magnetization seen following an inversion pulse and a $90^{\circ}$ pulse, unless one of the tissues is fat.

The signals can arise from: (i) uninverted positive magnetization detected with UTE or ZTE sequences from tissue with ultrashort $T_{2}$ components, (ii) positive short $\mathrm{T}_{1}$ fat magnetization and negative water magnetization at the time of the $90^{\circ}$ pulse, and (iii) positive shorter $T_{1}$ water magnetization and negative longer $T_{1}$ water magnetization at the time of the $90^{\circ}$ pulse.

It is possible to selectively null shorter or longer $T_{1}$ components to obtain exclusively in phase or out of phase 

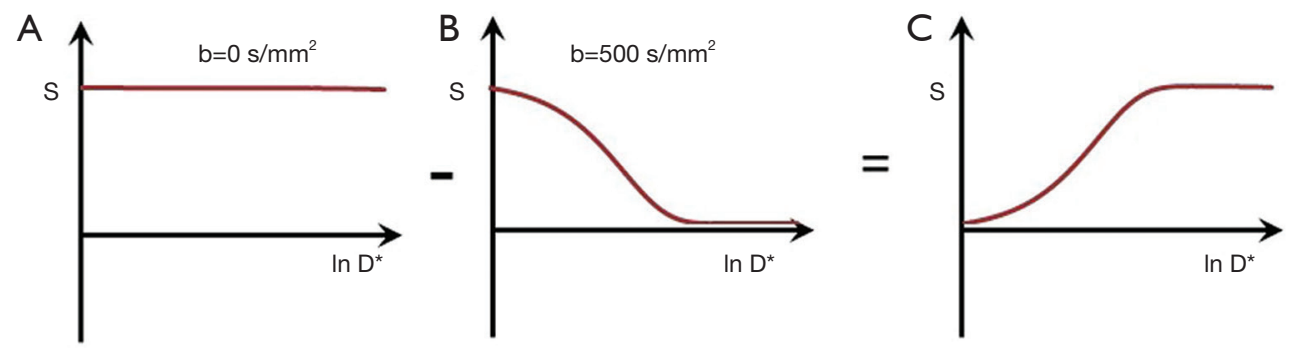

Figure 19 The $D^{*}$-filter for b values of " 0 " s/mm² and $1,000 \mathrm{~s} / \mathrm{mm}^{2}(A)$ and DS filter $\left(\mathrm{S}_{\mathrm{Do}}-\mathrm{S}_{\mathrm{D} 1000}\right)\left(\mathrm{b}=1,000 \mathrm{~s} / \mathrm{mm}^{2}-\mathrm{b}=0 \mathrm{~s} / \mathrm{mm} \mathrm{images}\right)(\mathrm{B})$. $\mathrm{S}$ is plotted against $\ln \mathrm{D}^{*}$ (univariate model). The $\mathrm{b}=0 \mathrm{~s} / \mathrm{mm}$ filter has zero slope and no $\mathrm{D}^{*}$-weighting. The $\mathrm{b}=1,000 \mathrm{~s} / \mathrm{mm}^{2} \mathrm{D}^{*}$-filter has a negative sequence weighting. Contrast is maximized where $b^{*}=1$ with this filter. Subtraction of the $b=1,000 \mathrm{~s} / \mathrm{mm}^{2}$ filter from the $b=0 \mathrm{~s} / \mathrm{mm}^{2}$ filter reverses the sequence weighting so that an increase in $\mathrm{D}^{*}$ increases the signal on the subtracted $\mathrm{D}^{*}$-filter (B). This can be used to make $D^{*}$ contrast synergistic with the contrast produced by increases in $\rho_{m}$ and $T_{2}$ when $D^{*}$ is increased.
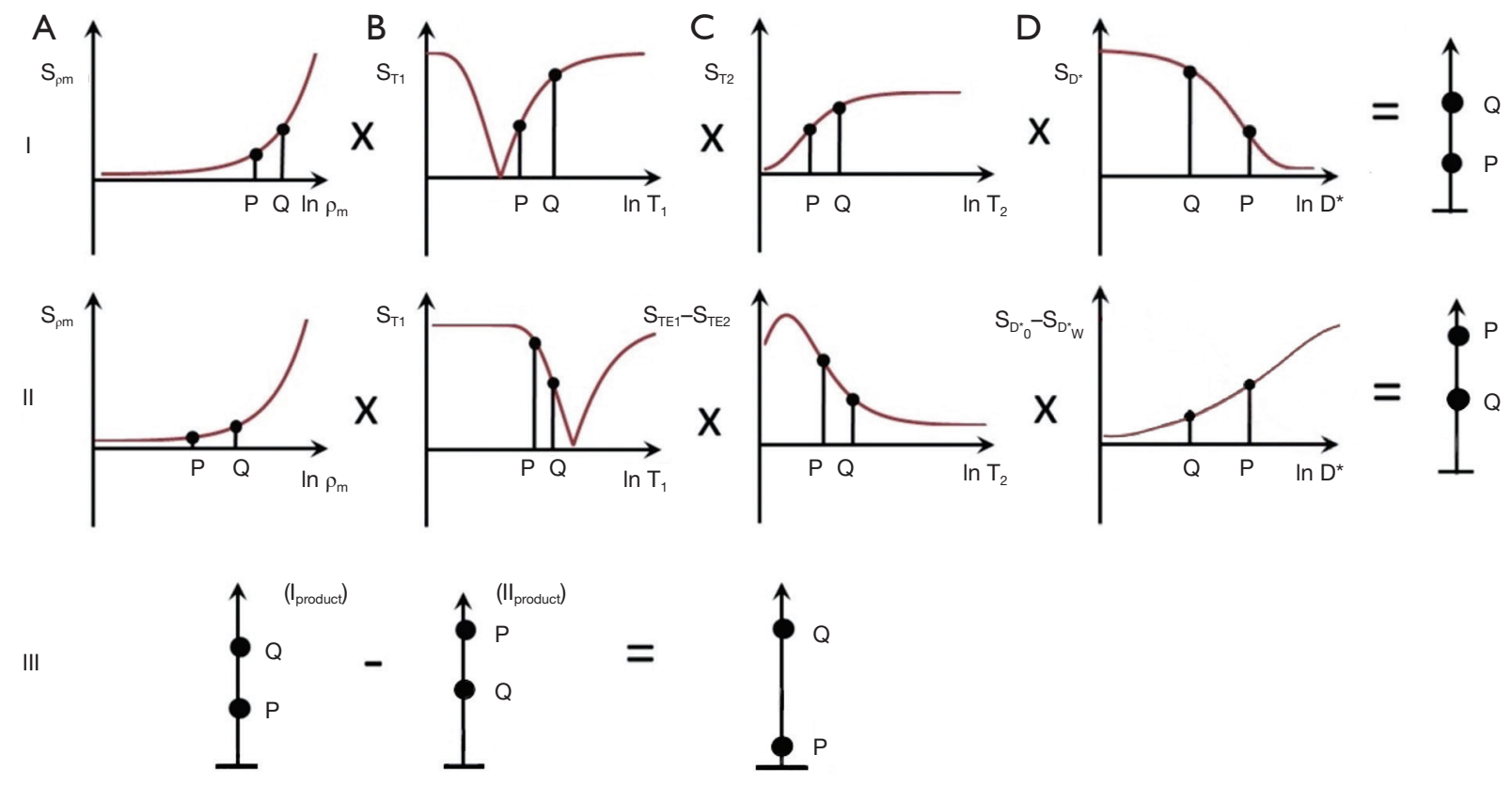

Figure 20 The subtracted IR diffusion echo subtraction (SIRDES) sequence for an increase in $\rho_{\mathrm{m}}, \mathrm{T}_{1}$, and $\mathrm{T}_{2}$ with a decrease in $\mathrm{D}^{*}$ from $\mathrm{P}$ to Q. The TP-filters for the sequence are shown in the top (I) and middle (II) rows. In the top row, a short TIs is used so that increases in $\rho_{\mathrm{m}}$, $\mathrm{T}_{1}$, and $\mathrm{T}_{2}$ with a decrease in $\mathrm{D}^{*}$ from $\mathrm{P}$ to $\mathrm{Q}$ result in positive contrast for each of the four filters, and the product of the signals (far right) shows positive contrast from $\mathrm{P}$ to $\mathrm{Q}$. In the middle row (II), with an intermediate $\mathrm{TI}_{\mathrm{i}}$ an increase in $\mathrm{T}_{1}$ from $\mathrm{P}$ to $\mathrm{Q}$ results in negative contrast. An $\mathrm{ES}$ $\mathrm{T}_{2}$-filter is used to produce negative contrast for an increase in $\mathrm{T}_{2}$ from $\mathrm{P}$ to $\mathrm{Q}$. The $\mathrm{DS} \mathrm{D}^{*}$-filter also shows negative contrast for a decrease in $\mathrm{D}^{*}$ from $\mathrm{P}$ to Q. Multiplication (far right) shows negative contrast from $\mathrm{P}$ to $\mathrm{Q}$. In the bottom row (III) subtraction of the product in row II from the product in row I increases the positive contrast from $\mathrm{P}$ to $\mathrm{Q}$ even further compared to that in row $\mathrm{I}$ alone.

images. It is also possible to selectively image tissues and fluid in phase and then out of phase, and add and subtract the two images to produce selective images of tissue alone and fluid alone (36). This technique has been used to show the brain without signal from CSF (37).
The large phase differences produced by differences in $T_{1}$ are a potential source of contrast for tissues that have longitudinal magnetizations near zero at the null point, but the amplitude of the magnetization may be small and the standard deviation of the phase is inversely proportional to 

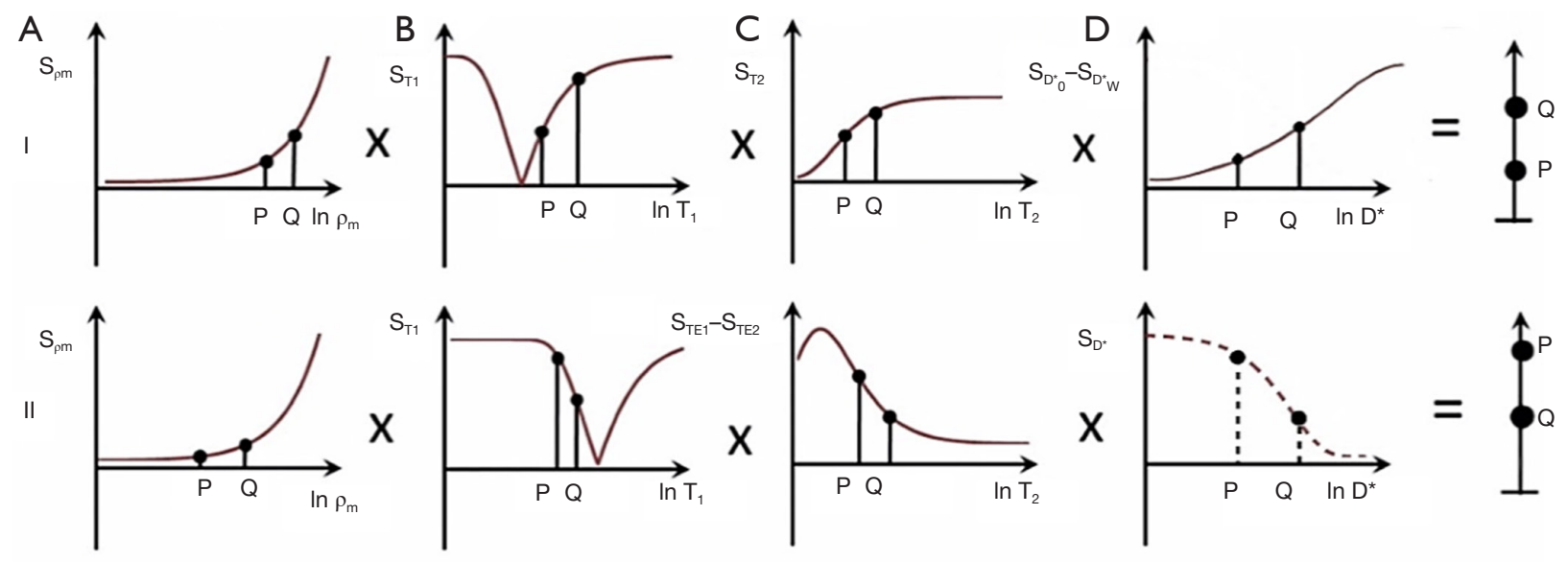

III
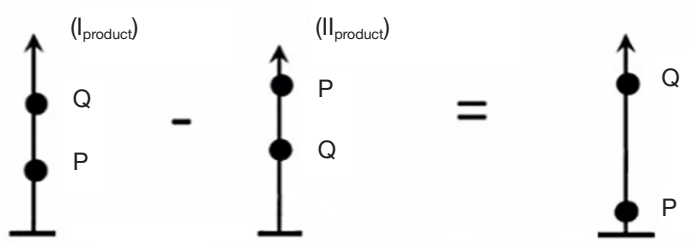

Figure 21 The SIRDES sequence for an increase in each of $\rho_{\mathrm{m}}, \mathrm{T}_{1}, \mathrm{~T}_{2}$ and $\mathrm{D}^{*}$ from $\mathrm{P}$ to $\mathrm{Q}$. In row $\mathrm{I}$ using a short $\mathrm{TI}_{\mathrm{s}} \mathrm{T}_{1}$-filter and $\mathrm{DS}$ filter, the increases in $\rho_{\mathrm{m}}, \mathrm{T}_{1}, \mathrm{~T}_{2}$ and $\mathrm{D}^{*}$ result in synergistic positive contrast from $\mathrm{P}$ to $\mathrm{Q}$ shown on the right. In row II, using an intermediate TIi T1-filter and ES the increases in $\mathrm{T}_{1}$ and $\mathrm{T}_{2}$ result in synergistic negative contrast from $\mathrm{P}$ to $\mathrm{Q}$ as shown on the right. However, it is not possible with the PGSE sequence to retain the negative $\mathrm{D}^{*}$ weighting and reverse the $\mathrm{T}_{2}$ contrast since this would require a heavily diffusion weighted sequence with an ultrashort or short TE. The $\mathrm{D}^{*}$ filter is therefore only shown in dashed line form. In row III, subtraction of the product in row II from the product in row I results in higher positive contrast from $\mathrm{P}$ to $\mathrm{Q}$ than in row I alone.

the magnitude signal to noise ratio (SNR). As a result, the phase image around the null point may be noisy.

Magnitude and phase IR mages with the same TI from the same acquisition can be multiplied together to improve gray white matter contrast in imaging the brain by exploiting the susceptibility difference between the two tissues (38).

\section{Fitting}

The term fitting is applied to the use of a model to relate the signal from two or more images The images are usually acquired in $\mathrm{T}_{1}$ dependent preparation periods typically $\mathrm{TI}$, or the $\mathrm{T}_{2} / \mathrm{T}_{2}{ }^{*}$ dependent period and are usually used to create maps.

In the TI period they include: (i) fitting of images acquired with the Look-Locker sequence or its variants using multiple TIs with single or multiple exponentials (39), and (ii) sliding radial acquisitions with progressively increasing TIs in the acquisition window [e.g., MPnRAGE and DESIRE (Double Echo Sliding IR)], as well as MT (e.g., with and without additional off-resonance rf pulses) preparations.

With an IR sequence after the $90^{\circ}$ pulse during the TE period two or more images may be acquired for multi-echo $\mathrm{T}_{2} / \mathrm{T}_{2}{ }^{*}$ modelling of single or multiple $\mathrm{T}_{2} / \mathrm{T}_{2}{ }^{*}$ components. This can be applied to the particular case of imaging fat and water tissue components using IDEAL or LIPO-QUANT (Liver Imaging of Phase-interference related signal Oscillation and QUANTification) for example $(40,41)$.

Contrast enhancement is usually modelled using the same acquisition at different times after contrast administration.

\section{Magnetization transfer (MT) (typically SE, GE dcs)}

With the IR sequence MT pulses can be applied before the inversion pulse during TR, after the inversion pulse during TI, or during both periods. Using basic modeling approaches these pulses produces a reduction in the observed $\rho_{\mathrm{m}}$ in the free pool and an equal fractional reduction in its observed $T_{1}(42)$. 


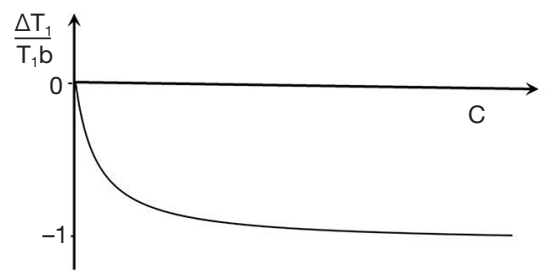

Figure 22 Plot of fractional change in $T_{1}\left(\Delta T_{1}\right)$ over baseline $T_{1}$ $\left(\Delta \mathrm{T}_{1} / \mathrm{T}_{1 \mathrm{~b}}\right)$ against concentration of contrast agent, c (e.g., GdDTPA). The fractional change in $T_{1}$ is negative and asymptotically approaches -1 at high concentrations of $\mathrm{c}$.

Fat and fluids display little or no MT effect so these typically cancel out in images produced from MT subtraction (MTS) of images without MT minus those with MT.

Disease generally reduces MT effects. When MT pulses are applied this results in diseased tissues showing a relative increase in their detectable $\rho_{\mathrm{m}}$ and observed $T_{1}$ with respect to the normal tissue. These effects may be made synergistic with the increase in $\rho_{\mathrm{m}}, T_{1}$ and $T_{2}$ usually seen in disease by use of SIR sequences.

The MTR ratio (MTR) (MT without $\left[\mathrm{MT}_{\mathrm{o}}\right]$ minus MT with $\left[\mathrm{MT}_{\mathrm{w}}\right]$ divided by $\mathrm{MT}_{\mathrm{o}}$ ) may be negative i.e., a net gain in signal with the IR sequence or positive with a net loss in signal. There are difficulties with the denominator for the MTR at the null point of IR sequences and it may be more useful to employ MTS rather than MTR, or normalize the MTR by making the denominator the square root of the sum of the squares of the signals with and without MT pulses.

MT effects may also arise incidentally from the use of off-resonance pulses in multi-slice IR imaging (43) and the use of fat saturation pulses (44). The variation between slices seen with multislice MT can be avoided with 3D acquisitions.

\section{Susceptibility $(\chi)$}

Susceptibility effects can be used with IR sequences to achieve $T_{1}$ contrast synergy with $T_{2}{ }^{*}$ effects. When the same agent on process decreases $T_{2}{ }^{*}$ it may also concurrently decrease $T_{1}$; likewise, there may be parallel increases in $T_{1}$ and $T_{2}{ }^{*}$ when susceptibility effects are reduced.

A magnitude reconstructed STIR sequence can be used to decrease signal resulting from a decrease in $T_{1}$ and a reduction in $T_{2}$ so that both effects are synergistic.

A synergistic increase in signal can be achieved with an intermediate TI and ES of a later echo from an earlier (e.g., UTE one) as with magnetic iron oxide particles (MIOPs) (45) and manganese enhancement (46).

Susceptibility phase images can be multiplied by IR magnitude images of the brain to increase contrast between gray and white matter (38).

FLAIR images can also be multiplied by susceptibility weighted images acquired with a GE sequence to show lesions and veins simultaneously in MS as FLAIR* (47).

\section{Contrast agents}

The comments below mainly relate to the clinical use of Gd-DTPA and magnetic iron oxide particle (MIOP) based agents. These agents produce a reduction in $T_{1}, T_{2}$ and/or $\mathrm{T}_{2}{ }^{*}$ (e.g., Figure 22) which is the opposite to the increase in $\mathrm{T}_{1}, \mathrm{~T}_{2}$ and $\mathrm{T}_{2}{ }^{*}$ commonly seen in disease.

(i) IR sequences can be subtracted before and after contrast administration to selectively show enhancement.

(ii) Reversed subtraction $\left(\mathrm{mTI}_{\mathrm{i}}-\mathrm{mTI}_{\mathrm{s}}\right) \mathrm{rSIR}$ sequences provide augmented $\mathrm{T}_{1}$ sensitivity and can be used to detect $T_{1}$ shortening due to contrast agents in the $T_{1}$ transition band of these sequences and show this as an increase in signal (Figure 14).

(iii) It is possible to make the $T_{1}$ shortening effect of contrast agents synergistic with the $\mathrm{T}_{2} / \mathrm{T}_{2}$ * shortening effect as with MIOPS by subtracting a later $\mathrm{TE}_{2}$ image from an earlier image at $\mathrm{TE}_{1}(\mathrm{ES})$. This reverses the sign of the sequence weighting of the $\mathrm{T}_{2}{ }^{*}$ filter of the SIRGES (SIR Gradient Echo Subtraction) sequence.

(iv) The range of detection of $\mathrm{T}_{1}$ and/or $\mathrm{T}_{2}$ shortening can also be extended by using UTE or similar sequences to detect signal even when the $\mathrm{T}_{2}$ and/or $\mathrm{T}_{2}{ }^{*}$ of the tissue or fluid of interest is greatly shortened by the contrast agent.

(v) The approach is similar for the detection of iron using the reversed rSIRGES Gradient Echo Subtraction and the gradient echo form of Diffusion (SIRDGES).

(vi) In general terms, the $T_{1}$ sequence weighting increases from T1-weighted SGE to T1-weighted SE to IR-FSE or MP-RAGE and then to rSIRES.

(vii) Gd-DTPA may produce a very large reduction in $\mathrm{T}_{1}$ e.g., in blood and in many tissues where it is present in relatively large concentrations. In these 
circumstances obvious contrast enhancement may be seen with less $T_{1}$-weighted sequences such as the T1-weighted SGE and so can be used effectively for fast Dynamic Contrast Enhancement.

(viii) The rSIRES sequence is generally slower than other $T_{1}$-weighted sequences but has higher $T_{1}$ weighting and may be useful in situations where double or treble dose contrast with or without a delay has been necessary to visualize enhancement or to make it more obvious. This includes some lesions in multiple sclerosis and some metastases in the brain. In other situations rSIRES may reveal contrast enhancement in apparently nonenhancing lesions such as low grade gliomas and Alzheimer's Disease.

\section{White adipose tissue (WAT) and brown adipose tissue (BAT). Yellow and red bone marrow [typically GE, Dixon fat water separation or suppression (FWSS) dcs]}

As outlined in the introduction, the STIR sequence was used to null the signal from fat in 1985 . Soon afterwards spectrally selective inversion pulses tuned to the fat frequency (but not that of protons in water) were used to selectively invert and null the signal from fat but not that of water in the form of SPIR, SPAIR and SPECIAL sequences.

In addition to techniques designed to suppress the signal from fat, other techniques have been used to separate the signals from fat and water so that they can be specifically imaged. The first of these used the basic Dixon method in which asymmetric SE or GE images are obtained with two TEs selected so that the fat and water signals are in and out of phase (48). Subtraction and addition of these images provide selective fat and water images. More advanced Dixon techniques use three points and other modifications (49). Further advances included IDEAL (Iterative Decomposition of water and fat with Echo Asymmetry and Least squares estimation (40) and LIPO-QUANT) (41) where multiple echoes are obtained, correction is made for differences in $T_{1}$ and $T_{2}{ }^{*}$ between fat and water signals and knowledge of the amplitude as well as $T_{1}$ and $T_{2}$ of different fat peaks (besides that of $-\mathrm{CH}_{2}$ ) is incorporated.

There is considerable interest in imaging metabolically active Brown Adipose Tissue (BAT) and separating it from much more abundant storage White Adipose Tissue (WAT) in humans. FDG-PET images of BAT reflect its metabolic activity and are amongst the most dramatic in medicine, but the use of radioactivity is a major restriction for studies involving children, populations, volunteers and follow up examinations.

Considerable effort has therefore been expended in developing MR techniques of different types $(50,51)$. In humans the most widely used method has been IDEAL Proton Density Fat Fraction (PDFF) imaging where WAT is recognised by virtue of the fact that its PDFF is typically $90 \%$ or above, whereas BAT typically only has a PDFF of about $30-70 \%$. The rest of BAT consists of mitochondrial rich water tissue with similarities to skeletal muscle. The main problem is that partial volume effects between WAT and muscle (and other water containing tissues) may simulate BAT since these effects have PDFFs in the BAT range. Considerable radiological skill may be needed to distinguish them from BAT. This has put a premium on MR techniques that can selectively display BAT and distinguish it from WAT. This is not a problem in rodents where WAT is in high concentration and is mainly located separately from WAT, but in adult humans BAT is generally in much lower concentration, widely dispersed and often mixed with WAT.

MASTIR sequences can be used to address this problem in several ways. The T1s of fat in WAT and BAT have been described as very different (e.g., $341 \mathrm{~ms}$ for WAT, and $460 \mathrm{~ms}$ for BAT at $3 \mathrm{~T}$ ) (52), and very similar (e.g., $280 \mathrm{~ms}$ for the WAT $-\mathrm{CH}_{2}$ peak and $278 \mathrm{msec}$ for the BAT $-\mathrm{CH}_{2}$ peak at 3T) (53).

\section{STIR echo subtraction (STIRES) (TI FANW $\left.\left[T E_{\text {in }}-T E_{\text {out }}\right]\right)$ (using $D X d c$ )}

Assuming that the $\mathrm{T}_{1}$ of fat in WAT is shorter than that of fat in BAT, it is possible to use $\mathrm{TI}_{\text {WATN }}$ to selectively null the fat in WAT so that the only remaining fat signal is that from BAT. This BAT fat can then be separated from water using the two point Dixon approach with subtraction of an in-phase image at $\mathrm{TE}_{\text {in }}$ from an out-of-phase one at $\mathrm{TE}_{\text {out }}$ to provide an exclusive image of BAT fat. This technique has been described as "dual echo inversion recovery" by Holstila et al. (54) and has been successfully performed by these authors in rats and human volunteers.

\section{Fat saturated SIR (FSSIR) $\left[T I_{\text {WATN }} \times\left(m T I_{s}-m T I_{i}\right)\right](G E$, asymmetric $S E, d c$ )}

Assuming the TIs of fat in WAT and BAT are the same, it 
is possible to spectrally suppress the fat signals from the fat in both WAT and BAT tissue with a single $\mathrm{TI}_{\mathrm{WATN}}=\mathrm{TI}_{\mathrm{BATN}}$ and then use a $\mathrm{TI}_{\mathrm{s}}$ to null BAT water and a $\mathrm{TI}_{\mathrm{i}}$ to null muscle water. These images can be subtracted to produce an image in which BAT water is higher signal (positive) and muscle water is lower signal (negative). (The water in WAT is only present in very low concentration.)

\section{Yellow and red bone marrow}

Yellow bone marrow can be imaged like WAT and the fat signal can be suppressed with $\mathrm{TI}_{\text {WATN }}$ or $\mathrm{TI}_{\mathrm{u}}$ to reveal normal water containing tissues such as subchondral veins, and abnormal water containing features such as bone marrow edema. Red bone marrow includes fat in significant proportions (e.g., 30-50\%), soft tissue which contains iron, and blood. These tissues and fluids are subject to susceptibility effects from trabecular bone. The main clinical interest is often primary or secondary malignancy in the soft tissue.

A STIR sequence suppresses fat (WAT) but leaves signal from water containing soft tissue, and from blood which is usually higher signal than the soft tissue. Addition of diffusion weighting provides synergistic $\rho_{\mathrm{m}}, \mathrm{T}_{1}$ and $\mathrm{T}_{2}$ contrast with diffusion contrast providing that $\Delta \mathrm{D}^{*}$ is negative. The signal from blood is reduced by the diffusion weighting and its high $D^{*}$. The synergistic $T_{1}$ contrast can be increased by use of a SIRES sequence instead of STIR. Reversal of $\mathrm{D}^{*}$ contrast by diffusion subtraction (DS) is an option when $\mathrm{D}^{*}$ is increased.

Fat suppressed DESIRE-UTE can be used to selectively detect ultrashort $T_{2}$ components associated with iron accumulation.

Subtraction of MT (without minus with MT) reduces the fat and blood signals leaving tissue signal. Abnormal tissue may show increased signal if its $M T$ effect is reduced. STIR MT sequences can make $\rho_{\mathrm{m}}, \mathrm{T}_{1}$ and $\mathrm{T}_{2}$ effects synergistic.

DIR sequences can be used to null fat and fluid and exploit any increased in the $T_{1}$ and $T_{2}$ in the water containing tissue component. This may be a useful option when $\mathrm{D}^{*}$ is increased.

\section{Ultrashort $\mathbf{T}_{2}$ tissues (typically UTE, ZTE dcs) (55)}

A key concept in this section is that conventional clinical pulse sequence inversion pulses do not fully invert the magnetization of tissues with $\mathrm{T}_{2} \mathrm{~s}$ of about the same duration as the pulse or less. Instead, the ultrashort $T_{2}$ tissue magnetization is saturated, partially saturated or partially inverted and its longitudinal magnetization recovers from a higher value than $-M_{Z}$. This may be of no consequence with conventional SE or GE des since the short or ultrashort $\mathrm{T}_{2}$ signals are not usually detected by them, but when UTE pulse sequences are used these signals can be detected and produce signal after the $90^{\circ}$ pulse.

Tissues may have a majority of short $T_{2}$ components (e.g., cortical bone) or a minority of short $\mathrm{T}_{2}$ components (as with many other tissues including myelin in the brain). The longitudinal magnetization of longer $T_{2}$ components is fully inverted by conventional $180^{\circ}$ pulses and $\mathrm{TI}_{\mathrm{s}}$ may be chosen to null this magnetization. After this it is possible to selectively visualize the signals from ultrashort $T_{2}$ tissue components.

Direct subtraction of a second echo image from the first (UTE) image may be used to detect ultrashort tissue $T_{2}$ components. However, if they are in low concentration (e.g., $3 \%$ ), a $2 \%$ decay in the $97 \%$ long $\mathrm{T}_{2}$ signal between components during the time between the first and second echoes may result in nearly $50 \%$ contamination of the subtracted image with long $T_{2}$ components and this is not detectable from the signal present in the second image.

However, if the long $T_{2}$ signal is nulled (as accurately as possible) and there is no signal on the second image this means that the ultrashort $T_{2}$ signal has decayed to zero and that long $T_{2}$ signal was very largely, or completely nulled at the first (UTE) echo. Even if it was not exactly nulled at the time of the first echo, and it had undergone $2 \%$ decay to reach zero signal at the time of the second echo, it means that it is a very minor contaminant not a $\approx 50 \%$ one.

\section{IR echo subtraction (IRES-UTE) [mTI $\left.I_{i}\left(T E_{u}-T E_{s}\right)-U T E\right]$}

A single inversion pulse can be used, followed by a first (UTE) echo and then a second echo (usually GE) with the second image subtracted from the first. Successful nulling is achieved when the signal on the second image is zero. In the brain, the $T_{1}$ of white matter varies with age, sex and site within the brain so that it may not be possible to achieve this throughout it with a single value of TI.

In the brain, for nulled long $\mathrm{T}_{2}$ components in white matter using $m$ reconstruction, subtraction of the TEs image from the $\mathrm{TE}_{\mathrm{u}}$ (UTE) image results in positive signal from the uninverted ultrashort $T_{2}$ components. For gray matter long $\mathrm{T}_{2}$ component longitudinal magnetization is negative at the long $T_{2}$ white matter nulling time, and the gray matter uninverted longitudinal magnetization 
ultrashort $T_{2}$ components are positive at this time leading to a net reduction in the transverse gray matter magnetization in $\mathrm{m}$ reconstructed images after the $90^{\circ}$ pulse. At TEs the gray matter ultrashort signal component is zero, and the long $T_{2}$ transverse signal is essentially unchanged from the TEu image, so the $\mathrm{m}$ reconstructed signal is larger than the TEu signal. Subtraction of the signal of the image at TEs from that at TEu gives a negative value. The difference between positive signal from white matter and the negative signal from gray matter produces high contrast between the two tissues on the subtracted image.

\section{Multiplied IR echo subtraction (MIRES) [ $\left(m T_{1} s \times m T I_{j}\right)$ $\left.\left(T E_{u}-T E_{s}\right)\right]$ and Short TR Adabatic pulse prepared IR (STAIR)}

A potential solution to the problem of nulling long $T_{2}$ components with different $T_{1} \mathrm{~s}$ is to use two inversion pulses to provide a wider range of $\mathrm{TI}_{s}$ for which the long $\mathrm{T}_{2}$ signal is successfully nulled. Another approach is to use a short TR (e.g., 140-150 ms) with a single optimized TI to give signal suppression for a broad range of TIs as the STAIR or STAIRES sequence.

\section{Double echo sliding IR (DESIRE-UTE) (31)}

Another approach is to use a sliding IR sequence which produces a range of $\mathrm{TI}_{\mathrm{s}}$. These can be chosen to cover the likely values of TI to null the long $T_{2}$ signal of the tissue of interest and ensure that the signal is zero on the second image for one or more $\mathrm{TI}_{\mathrm{s}}$. $\mathrm{TI}_{s}$ can be chosen to cover values for different tissues (e.g., both gray and white matter) as well as changes in $T_{1}$ in disease.

In disease an increase in $T_{2}$ of the ultrashort $T_{2}$ component means more magnetization is inverted (so the short $T_{2}$ component signal is reduced). If there is a concurrent increase in $T_{1}$, this also reduces signal. The longer $T_{2}$ of the short $T_{2}$ components also reduces the signal resulting from ES. These synergistic effects provide high sensitivity for increases in $T_{1}$ and $T_{2}$ in the ultrashort $\mathrm{T}_{2}$ range.

\section{Angiography (typically GE, SE, bSSFP dcs)}

Angiography can be performed with contrast agents in which there are large decreases in $T_{1}$ and $T_{2} / T_{2}{ }^{*}$, or without contrast agents when there are less marked differences in tissue or fluid properties between blood and tissues $(56,57)$ as described below.

Inflow of fully recovered blood into a slice of partially saturated blood leads to a shortening of blood $\mathrm{T}_{1}$. Inflow of negatively longitudinally magnetized blood produced by an inversion pulse leads to an increase in $T_{1}$.

Inflow of blood in excess of what is metabolically required may lead to less desaturation and a decrease in paramagnetic deoxyhemoglobin and so produce a net increase in $\mathrm{T}_{2}$.

It is possible to alternatively apply $180^{\circ}$ pulses outside of the slice to invert inflowing blood and not invert it producing contrast as a function of flow.

It is also possible to null the surrounding tissues to increase contrast between higher signal blood and tissue.

There are mechanisms which increase or maximise the signal from blood (e.g., inflow of fully recovered blood with flow compensation) as well as mechanisms that decrease signal from blood (e.g., dephasing in the presence of a gradient). Subtraction of the second case from the first produces synergistic high contrast from blood with suppression of background tissue signal.

Inversion pulses can also be used to produce black blood imaging of the heart and produce more widespread suppression of background tissue signal while maintaining that from blood [see previous section on (MIR)].

\section{Perfusion}

The same general considerations that apply to angiography apply to perfusion where signal is detected from the combination of blood and tissue, not just from blood alone. Contrast agents usually lead to relatively larger changes in tissues than non-contrast agent approaches.

Inversion and saturation pulses can be used upstream of a volume of interest to modify the signal of inflowing blood and the signal from this volume can also be modified. This is described under the general heading of Arterial Spin Labelling (ASL) $(58,59)$. A particular form of this technique employs inversion pulses alternatively applied and not applied to the upstream blood followed by subtraction of the two images from the volume of interest (Flow-sensitive Alternating IR [FAIR]) (60). This technique can also be combined with diffusion (61).

\section{Regional Cerebral Blood Volume (CBV), VAscular Space Occupancy (VASO)}

$\mathrm{CBV}$ is a measure of the blood content of tissue irrespective 
of its flow. Contrast agents can be used for CBV as well as non contrast agent methods exploiting the long $\mathrm{T}_{1}$ and/ or $\mathrm{T}_{2}$ of blood relative tissue and compared to the baseline signal with an inversion pulse. Vascular space occupancy is a technique in which the signal from blood is nulled with an inversion pulse $(62,63)$.

\section{Lymphography, cholangio-pancreatography, urography, cisternography and CSF mapping}

Subtraction IR sequences either singly or in combination can be used to suppress the signal from soft tissues as well as blood to selectively demonstrate lymph or interstitial fluid, bile, urine and CSF. The separation is based on differences in $T_{1}$ rather than exploiting the long $T_{2} s$ of these fluids.

\section{Functional MRI of the brain (fMRI)}

In the classical fMRI procedure activation is associated with increased blood flow leading to decreased saturation of deoxyhemoglobin, less susceptibility effect and an increase in $T_{2}$ and $T_{2}{ }^{*}$. This applies to venous blood and tissues as a consequence of the blood within them. The increased blood in tissue can lead to an increase in CBV (the fraction of blood within tissue, and so $\rho_{\mathrm{m}}$ ) and an increase in $T_{1}$ of the combined signal from tissue and blood. Increase in blood in tissue may lead to an increase in $\mathrm{D}^{*}$. The overall changes are similar to the common changes seen in disease of an increase in $\rho_{\mathrm{m}}, \mathrm{T}_{1}, \mathrm{~T}_{2}$ and $\mathrm{D}^{*}$ as well as increased vascular flow and perfusion. The usual data acquisition is with a heavily $\mathrm{T}_{2}{ }^{*}$ weighted EPI sequence, but it is also possible to use SIRGES or SIRDGES as for clinical lesions to produce synergistic contrast.

Inflow of fully recovered blood to a partially saturated blood and tissue produces a net shortening of $\mathrm{T}_{1}$, but the blood longitudinal magnetization can be inverted prior to inflow leading to an increase in $T_{1}$ to make it synergistic with the other tissue property changes.

\section{Quantification of MR tissue properties}

The use of two $\mathrm{TI}_{\mathrm{s}}$ and the use of two $\mathrm{TE}_{\mathrm{s}}$ with different sequences as well as different degrees of diffusion and MT weighting allows measurement of $\mathrm{T}_{1}, \mathrm{~T}_{2}, \mathrm{D}^{*}$ and MTRs at a basic level as part of a standard imaging protocol. Fat fractions can also be obtained, but accurate measurements of $\mathrm{T}_{1}, \mathrm{~T}_{2}, \mathrm{MT}$ and fat fractions require purpose designed data acquisitions.
Values of $T_{1}$ for nulling can be obtained by basic techniques or more advanced methods such as Look-Locker and fingerprinting (64).

The wider dynamic range of the SIR $T_{1}$-filter and SIR sequences with MT contrast may allow more sensitive measurement.

The difficulty in accurate measurements is incorporated in the way that tissue properties are described e.g., as "observed" and "apparent".

\section{Faster imaging}

The acquisition of MASTIR images is prolonged when it is necessary to obtain images with two TIs. This is much less so for two TEs, and not so for ps and $\mathrm{m}$ reconstructions.

The sequence TR, TI and "inversion" pulse angle can be reduced to produce faster images with appropriately adjusted nulling points but this is usually accompanied by a reduction in signal level.

Two inversion images with different TIs can be obtained from a single inversion pulse using the Look-Locker sequence but this is also accompanied by a reduction in SNR.

Multi-spoke acquisition as for UTE and other radial sequences can speed up sequences and produce a useful reduction in long $\mathrm{T}_{2}$ signals (65).

Radial sampling repeatedly samples the centre of k-space and this can be exploited to improve SNR in the region of most importance in determining contrast.

K-space sampling may follow a centric ordered pattern as used for contrast enhancement.

Increases in time efficiency are also possible with both Sensitivity Encoding (SENSE) and compressed SENSE data acquisitions.

\section{SNR and contrast to noise ratio (CNR)}

In general, the IR sequence is regarded as high contrast but low SNR. This may be exacerbated when two separate acquisitions are required (e.g., with two TIs). The trade-off of signal $v s$ contrast may be explicit as in Eqs. $[5,6]$.

The target tissue of interest may be in low concentration, e.g., short $T_{2}$ components in the brain, blood in many tissues and normal fat in the liver. Subtracted differences may also be small and there may only be small changes in tissue properties when subtle disease is being sought. These all tend to decrease SNR and CNR.

The nulling of signal may allow use of thicker slices. In 

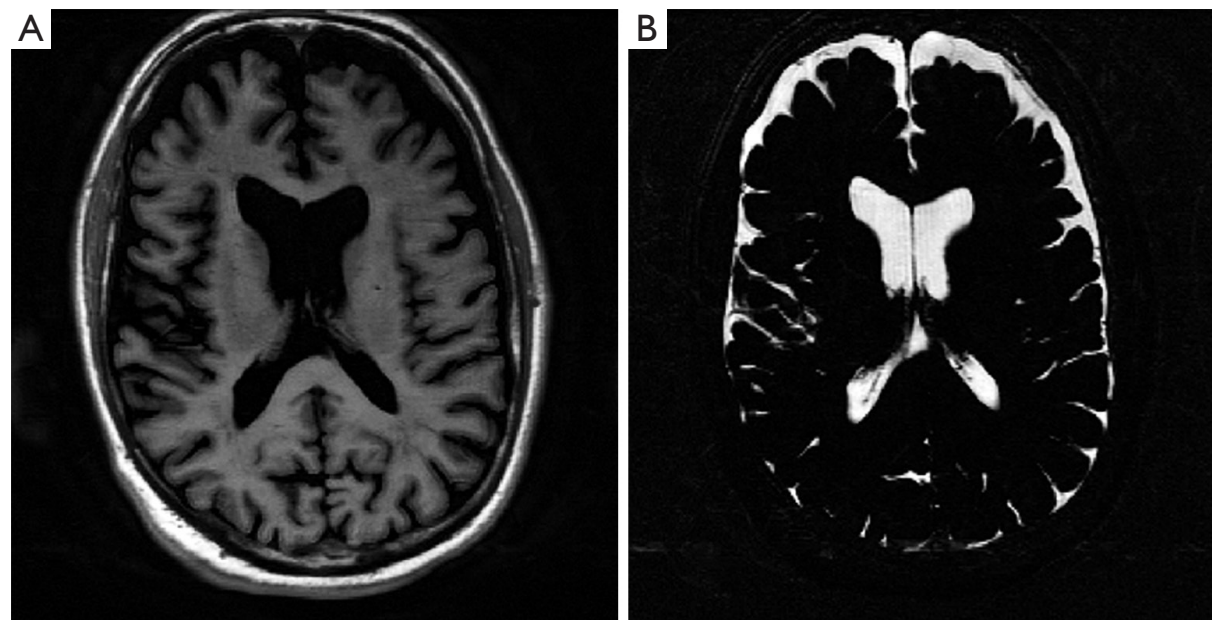

Figure $23 \mathrm{AIR}\left(\mathrm{ps} \mathrm{TI}_{\mathrm{i}}+\mathrm{mTI}_{\mathrm{i}}\right)(\mathrm{A})$ and $\mathrm{S}^{1} \mathrm{IR}\left(\mathrm{mTI}_{\mathrm{i}}-\mathrm{ps} \mathrm{TI}_{\mathrm{i}}\right)(\mathrm{B})$ images of the head in a normal subject. The brain is seen in (A) with zero signal from CSF. In (B) there is no signal from the brain and CSF shows high signal. The patterns follow those shown in AIR $\mathrm{T}_{1}$-filters (Figure 9) and the $\mathrm{S}^{1} \mathrm{IR}_{\mathrm{1}}$-filter (Figure 10). CSF, cerebrospinal fluid.

addition, some tissues such as cartilage are in layers with relatively little curvature in particular places and these may also allow the use of thicker slices without significantly increasing problematic partial volume effects.

It may also be possible to combine one image with a relatively high SNR with another of lower SNR which provides most of the contrast.

\section{Examples}

\section{AIR and SIR images (same initial TIs)}

Figure $23 A$ shows an AIR image with a TI of $1,200 \mathrm{~ms}$ at 3.0 T in which signal is seen from the brain and no signal is seen from CSF consistent with a low pass $\mathrm{T}_{1}$-filter as shown in Figure 9. Figure $23 B$ is the corresponding $S^{1} \mathrm{IR}$ image in which there is no signal from the brain but there is a high signal from CSF consistent with the high pass $T_{1}$-filter shown in Figure 10.

Figure 24 is a fat suppressed AIR-UTE image of the knee articular cartilage showing short and ultrashort $\mathrm{T}_{1}$ tissue. Short and ultrashort $T_{1}$ and $T_{2}$ components are highlighted within the deep layers of the cartilage. Longer $T_{1}$ tissues within cartilage and elsewhere as well as fluid signals have been suppressed.

\section{SIR (different TIs)}

Figure $25 \mathrm{~A}$ is a short $\mathrm{TI}_{\mathrm{s}}$ image in which white matter is nulled and gray matter is seen. Figure $25 B$ is an intermediate Tli image in which gray matter is nulled and white matter is seen. Figure $25 C$ is a subtraction image of (B) (intermediate $\mathrm{TI}_{\mathrm{i}}$ ) from (A) (short $\mathrm{TI}_{\mathrm{s}}$ ) showing high signal for gray matter, low signal for white matter and intermediate signal for CSF. Figure 25D is the reverse subtraction in which gray matter is low signal and white matter is high signal with CSF intermediate signal. With the SIR sequence the highest intracranial contrast is between gray and white matter, not between CSF and white matter as with conventional $T_{1}$ and $\mathrm{T}_{2}$-weighted images.

Figure 26 is a case of Multiple Sclerosis in which the same slice is shown with a $\mathrm{T}_{2}$-weighted $\mathrm{SE}$ sequence in (A) and a MSIR sequence in (B). In (A) lesions are seen as high signal in the central white matter which also shows some background slightly increased signal. CSF is high signal. In (B) many of the lesions appear larger. In addition, the two highest signal lesions in (A) have central low signal areas. There is extensive diffuse high signal throughout much of the central white matter which extends peripherally towards the $\mathrm{U}$ fibers in (B). High signal is also seen in areas at the gray white matter junction. CSF is zero signal. The mild background change seen in (A) is far more obvious and more extensive in (B).

Figure $27 \mathrm{~A}$ is a gray matter nulled $\left(\mathrm{TI}_{1}\right)$ image and Figure $27 \mathrm{~B}$ is a white matter nulled $\left(\mathrm{TI}_{\mathrm{s}}\right)$ image in a normal brain. Figure $27 \mathrm{C}$ is the (reverse) subtraction of (B) from (A) i.e., $\left(\mathrm{mTI}_{\mathrm{i}}-\mathrm{mTI}_{\mathrm{s}}\right)$. White matter is white, gray matter is black and CSF is gray. In (C) the $\mathrm{U}$ fibers in the posterior, frontal 

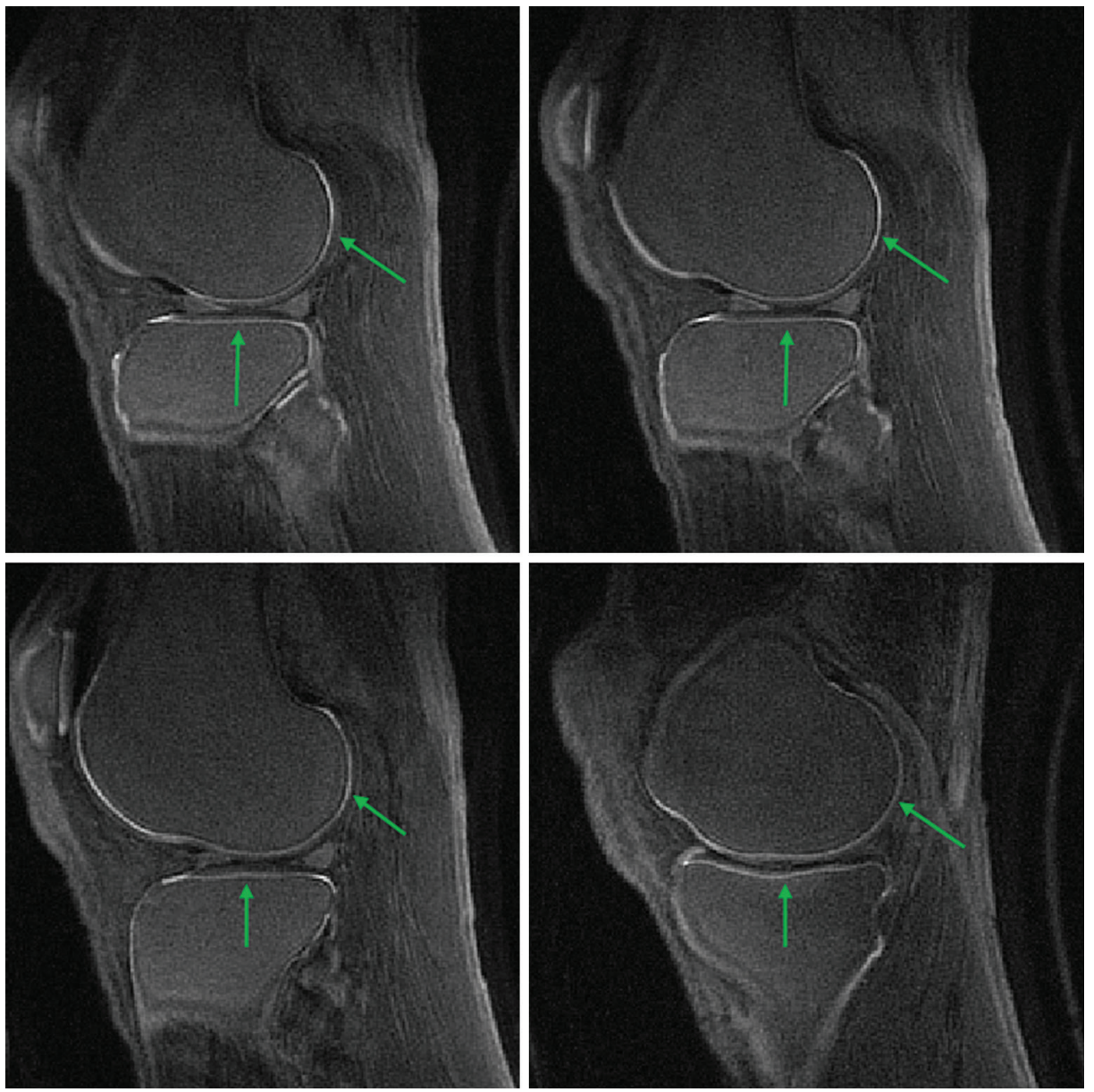

Figure 24 Fat suppressed AIR-UTE image of the knee. The short and ultrashort T1 deep and radial layers of the articular cartilage show high signal (arrows). The longer T1 tissue and fluid signals are suppressed.

and temporal lobes are well seen. These are not seen in (A) or (B).

Figure 28 is a gray matter nulled $\left(\mathrm{TI}_{\mathrm{i}}\right)$ image, $(\mathrm{B})$ is a white matter nulled $\left(\mathrm{TI}_{\mathrm{s}}\right)$ image of a normal volunteer who had had repeated Gd-DTPA administrations. (C) is the (reverse) subtraction $\left(\mathrm{mTI}_{\mathrm{i}}-\mathrm{mTI}_{\mathrm{s}}\right)$. The dentate nuclei (arrows in $\mathrm{C}$ ) are high signal consistent with marked shortening of $T_{1}$ due to gadolinium deposition. White matter in the temporal lobe is best seen in (C).

Figure 29 shows brain samples: normal (A), mild Alzheimer's Disease (AD) (B), and marked $\mathrm{AD}(\mathrm{C})$ all imaged with SIR $\left(\mathrm{mTI}_{\mathrm{s}}-\mathrm{mTI}_{\mathrm{i}}\right)$ sequences. The cortex is high signal and white matter is low signal in all three images. Layers are clearly seen within the cortex in the normal brain with the external layer high signal and layer IV low signal (A). The layers are less obvious in (B) and are indistinct in (C).

Figure 30 is of the median nerve tissue sample. (A) is a short TIs nulled image with the perineurium nulled, (B) is a higher $\mathrm{TI}_{\mathrm{i}}$ image with the endoneurium and nerve fibers nulled. (C) is the subtraction of (A) from (B). It shows a high signal from the perineurium and variable signals within nerve fasciculi which are not apparent on either (B) or (C).

\section{IR UTE and phase images}

Figure 31 is from a Multiple Sclerosis patient and shows

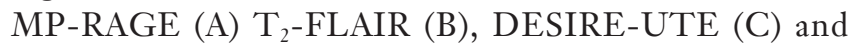
phase (D) images. Two lesions are seen as low signal on (A), high signal on (B), high signal on (C) and high signal on (D). The appearances are consistent with an increase in $\mathrm{T}_{1}(\mathrm{~A})$, increase in $T_{2}(B)$, loss of myelin (C) and phase reversal (D).

Figure 32 is a transverse forearm image showing the radius and ulnar. UTE-IR ES image with magnitude image (A) and phase image (B). The phase image shows high signal 

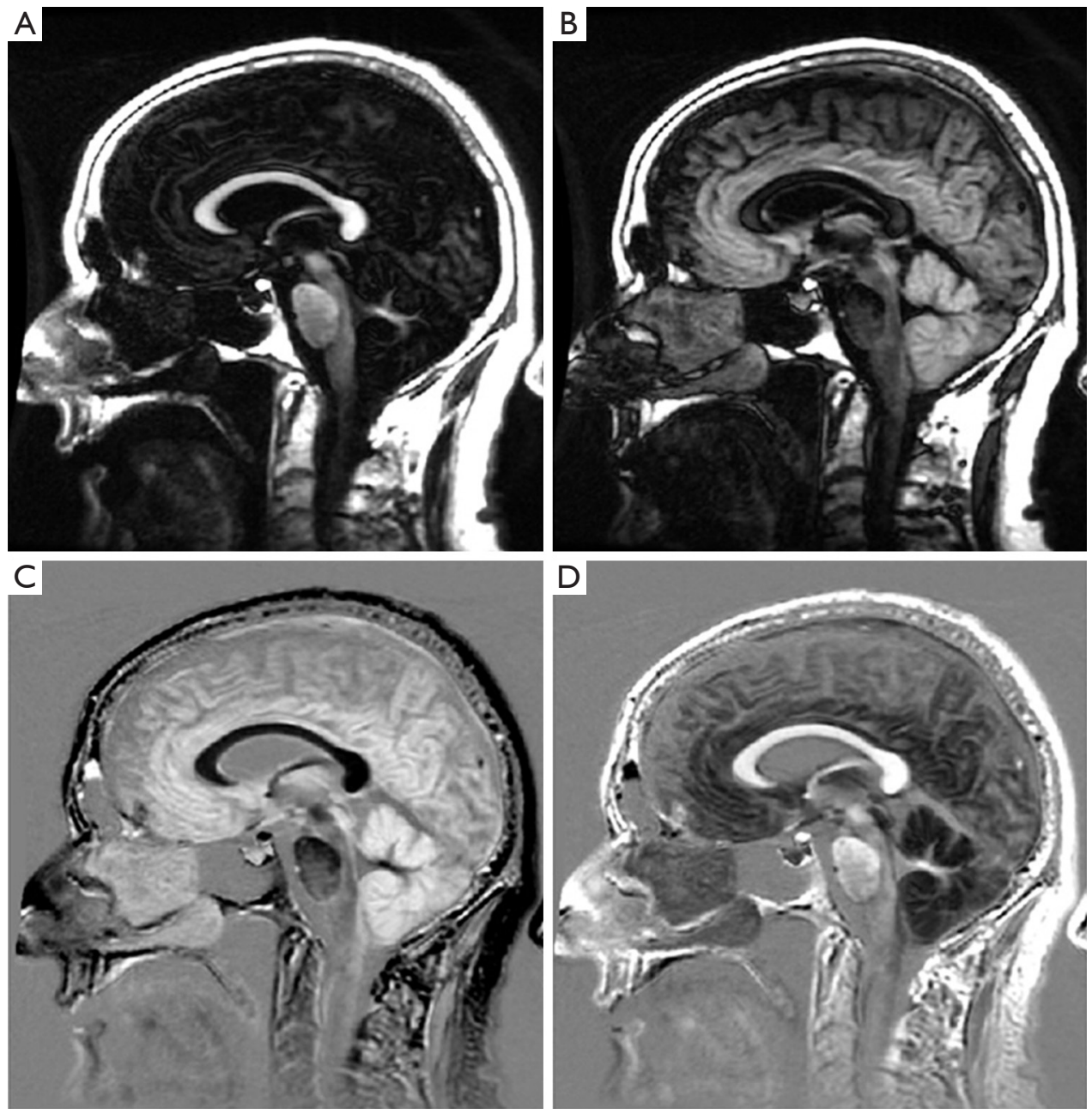

Figure 25 Sagittal images of a normal brain: mTIi nulling gray matter (A), mTIs nulling white matter $(B), \operatorname{SIR}\left(\mathrm{mTI}_{\mathrm{s}}-\mathrm{mTI}_{\mathrm{i}}\right)$ in $(\mathrm{C})$ and reversed SIR $\left(\mathrm{mTI}_{1}-\mathrm{mTI}_{\mathrm{s}}\right)$ in (D). There is high gray white matter contrast with gray matter high signal and white matter low signal in (C). In (D) the gray white matter contrast is reversed with white matter white, and gray matter black. CSF is of intermediate signal in both (C) and (D). CSF, cerebrospinal fluid.

within the cortex of both bones (arrows).

\section{Discussion}

\section{General}

The description above provides a framework for understanding different types of MASTIR sequences. These employ different inversion pulses, $\mathrm{TI}_{\mathrm{s}}, \mathrm{TE}_{\mathrm{s}}$, weightings (e.g., $\mathrm{D}^{*}, \mathrm{MT}$ ), data collections, reconstructions and/or processing and can be used to increase the sensitivity of the IR sequence to changes in $T_{1}$ by synergistic increase in $T_{1}$ contrast derived from one or more IR acquisitions. This contrast can be added to that produced by $\rho_{\mathrm{m}}, \mathrm{T}_{2}$ and $\mathrm{D}^{*}$ for the common changes (increases) seen in disease as well as decreases in $T_{1}, T_{2}, T_{2}{ }^{*}$, and/or $D^{*}$ produced by disease, iron deposition and contrast agents. The sequence can also be used to highlight or suppress the signal from specific tissues and/or fluids.

There are other combinations of IR sequences not dealt with in this paper such as weighted averaging of IR images with different $\mathrm{TI}_{\mathrm{s}}(65)$ and $\mathrm{T}_{1}$ Rho And Magnetization transfer and INvErsion Recovery (TRAMINER) (66).

\section{Pulse sequences as TP-filters}

The TP-filters approach provides a method of visualizing 

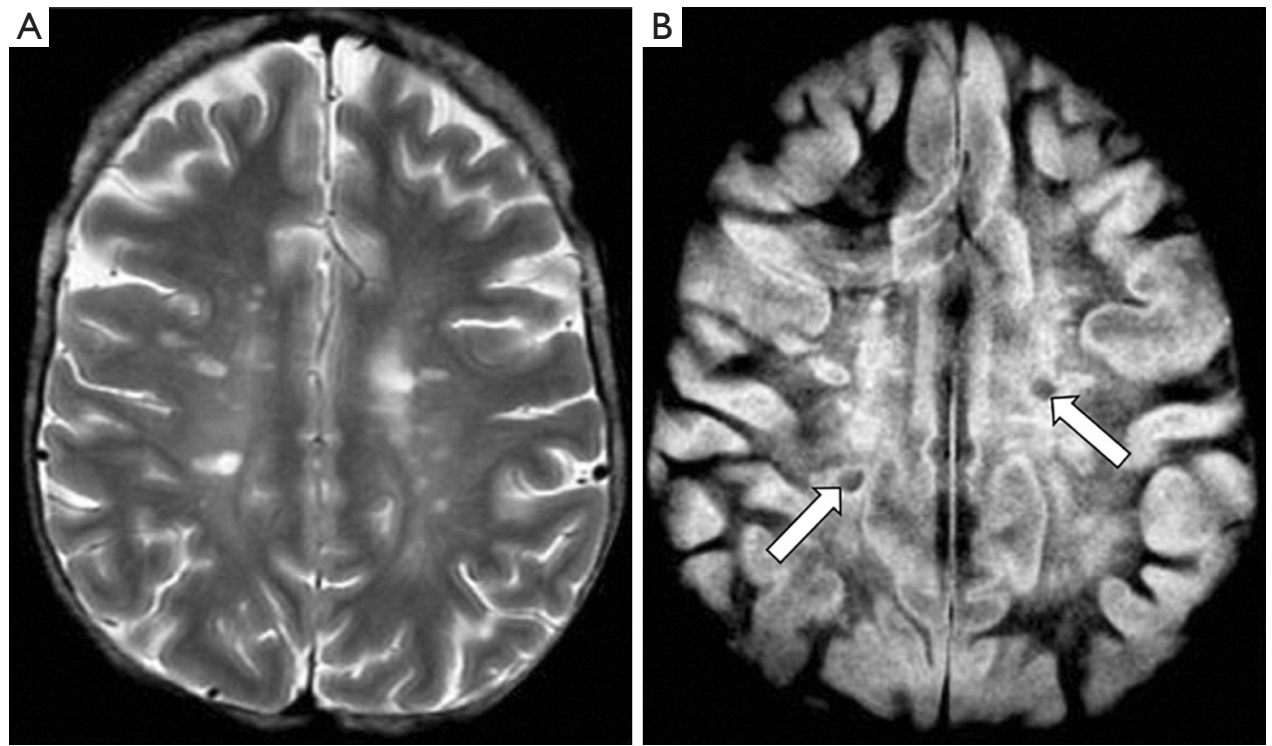

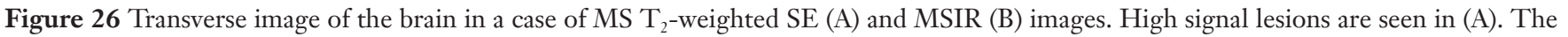
two with highest signal appear dark in (B) (arrows). Other lesions appear more extensive in (B). There is a mild increase in signal centrally in (A). This is much more marked in (B) where it extends towards the peripheral U fibers in much of the white matter. The MSIR image shows much higher contrast than the $\mathrm{T}_{2}$-weighted SE image for this abnormality. CSF is zero signal in (B). CSF, cerebrospinal fluid.
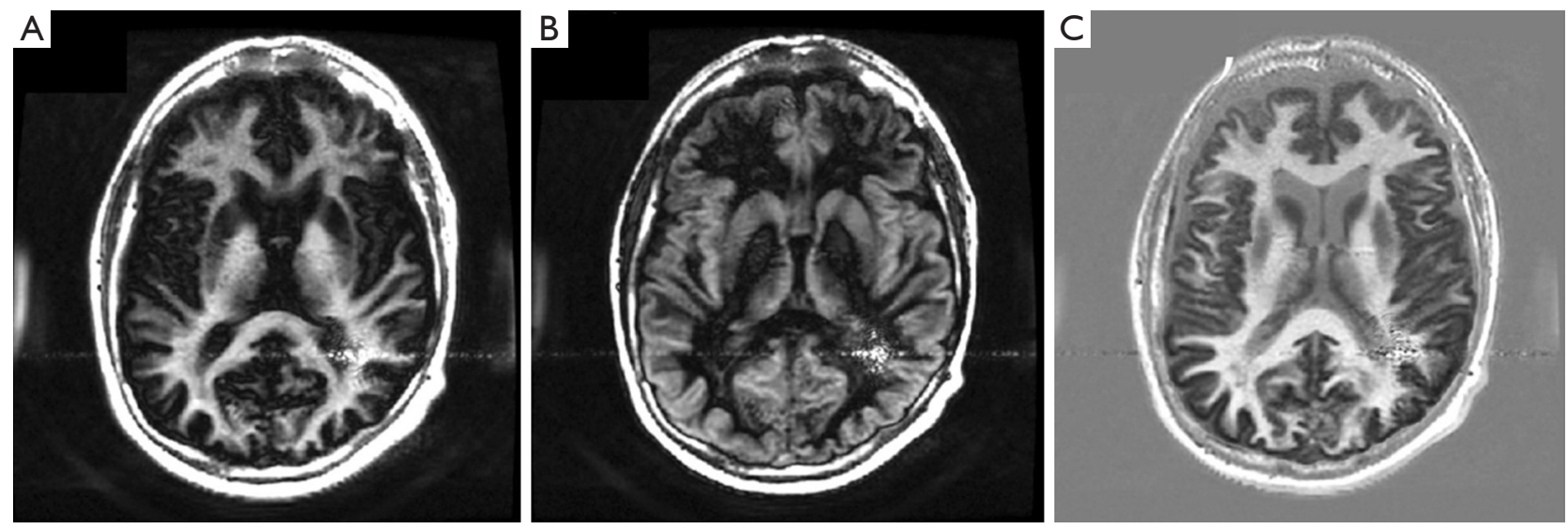

Figure 27 Brain $\left(\mathrm{mTI}_{1}-\mathrm{mTI}_{\mathrm{s}}\right)$ reversed subtraction. Gray matter is nulled in (A) with TIi and white matter is nulled in (B) with TIs subtraction of (B) from (A) gives (C). The $\mathrm{U}$ fibers in the posterior frontal and temporal lobes are seen more clearly in (C) than in (A) or (B).

the image signal, contrast and weighting produced by pulse sequences from tissues and fluids with different $M R$ properties. The filters show sequence weighting as the slope of the filter, and image weighting as the overall contribution to contrast from sequence weighting multiplied by change in tissue properties for each tissue property.

The TP-filters show whether contrast produced by a difference or change in one tissue property is synergistic or opposed to that produced by difference or change in another tissue property.

The effects of multiplication, addition and subtraction which may be different over particular ranges of tissue property values are well shown with this approach. More advanced features dealing with issues such as fractional contrast, and other types of pulse sequences are dealt with elsewhere (12). 

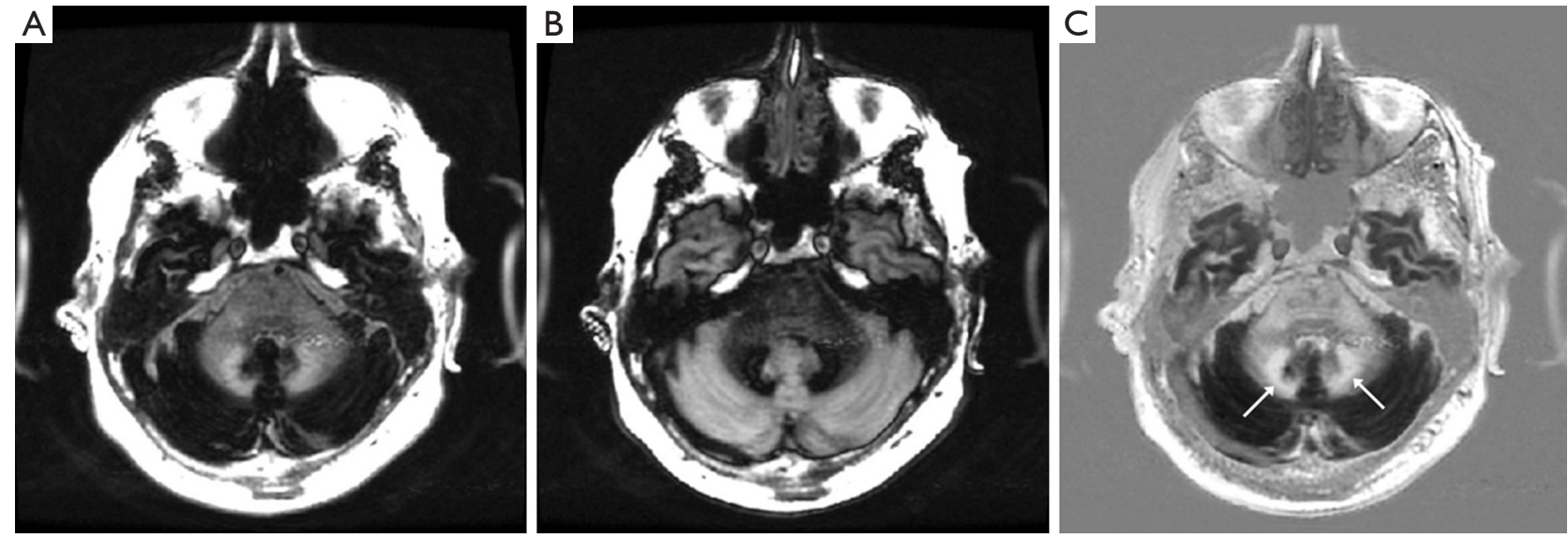

Figure $28 \mathrm{rSIR}\left(\mathrm{mTI}_{\mathrm{i}}-\mathrm{mTI}_{\mathrm{s}}\right)$ reversed subtraction image in a normal subject after multiple administrations of intravenous Gd-DTPA. (A) is a gray matter nulled image $\left(\mathrm{TI}_{\mathrm{i}}\right),(\mathrm{B})$ is a white nulled image $\left(\mathrm{TI}_{\mathrm{s}}\right)$ and $(\mathrm{C})$ is the reversed subtraction $\mathrm{rSIR}\left(\mathrm{mTI}_{\mathrm{i}}-\mathrm{mTI}_{\mathrm{s}}\right)$ image. The dentate nuclei are high signal in (C) (arrows). White matter is best seen in the temporal lobes in (C).
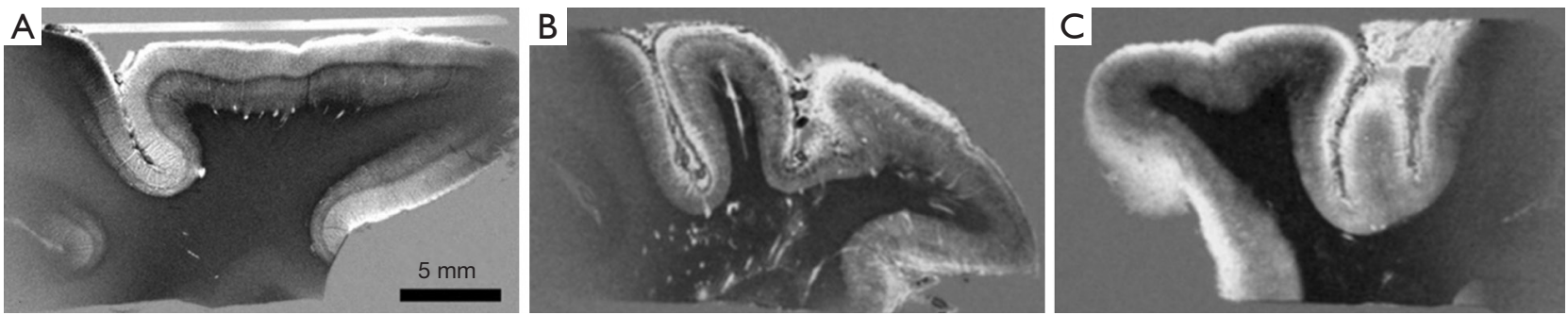

Figure 29 Normal (A), mild AD (B) and marked AD (C) SIR images of cortical brain tissue samples obtained at 11.7T. Cortical layers are well seen in (A), less distinct in (B) and are not well seen in (C).
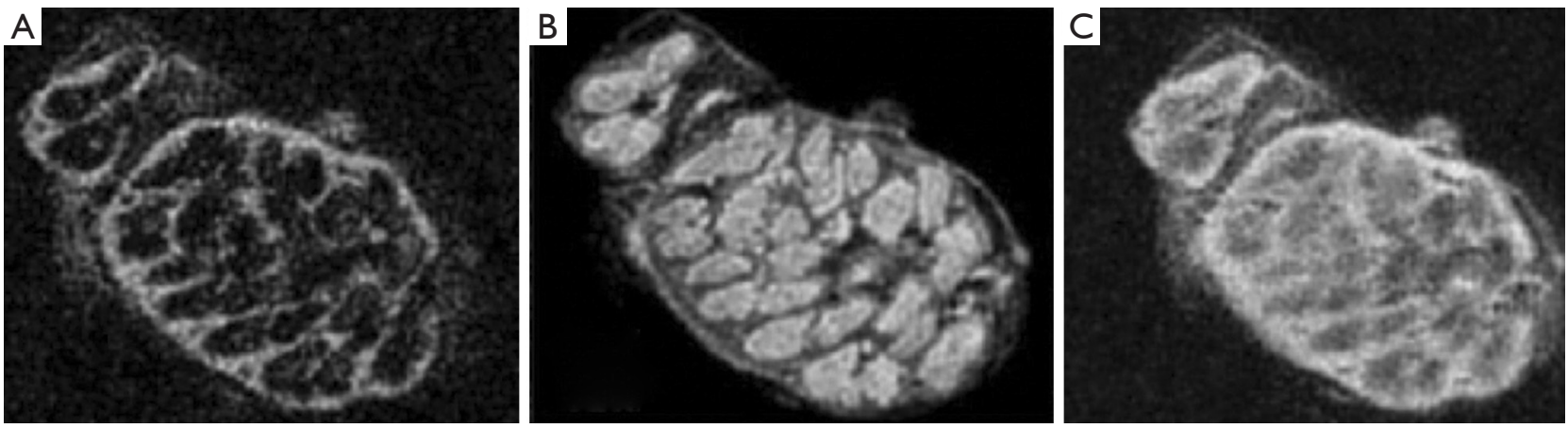

Figure 30 Median nerve tissue sample images with short TIs (A), intermediate TIi (B) and subtraction (A,B) in (C). The perineurium is seen in (A) and the endoneurium and nerve in (B). Subtraction (C) shows variation in the fascicular signal which is not apparent on (A) or (B). 

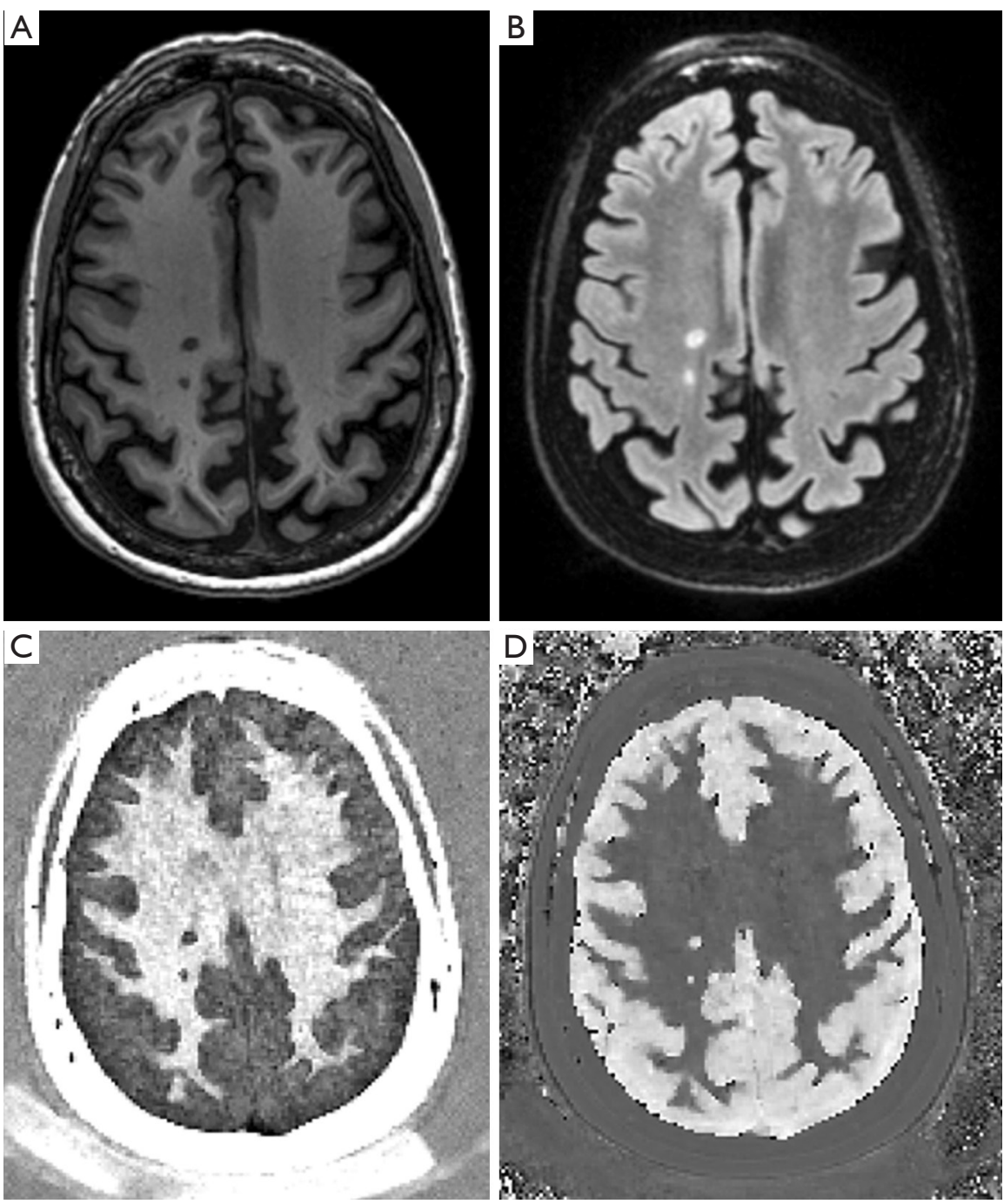

Figure 31 MP-RAGE (A), T -FLAIR (B), ES IR-UTE magnitude (C) and phase image (D). Two lesions are seen as low signal due to increase in $\mathrm{T}_{1}(\mathrm{~A})$, high signal due to an increase in $\mathrm{T}_{2}(\mathrm{~B})$, low signal due to loss myelin in $(\mathrm{C})$ and high signal due to phase reversal in (D).
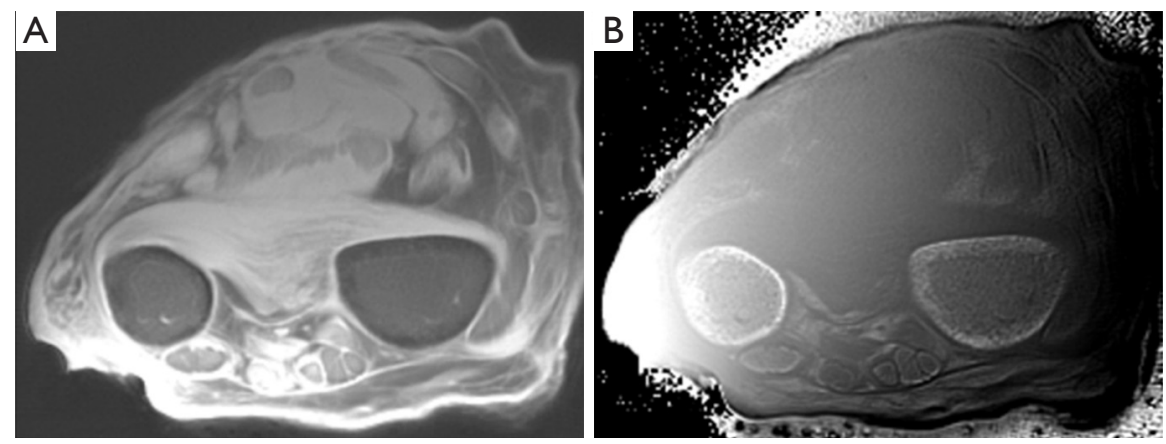

Figure 32 IR UTE transverse image of the forearm: magnitude (A) and phase (B) images showing the radius and ulnar. The phase image (B) shows high signal in cortical bone. 


\section{Fat and fluid signal suppression and separation}

In addition to the methods of fat signal suppression and separation already mentioned it is possible to perform asymmetric adiabatic pulse to excite fat but not water (67). Fat suppression can also be achieved with minimal saturation of water when performing UTE sequences using a soft-hard comparative rf pulse (68).

In the preceding text methods of selectively suppressing long $T_{1}$ and $T_{2}$ water signals from fluids have been described. These include the use of inversion pulses, subtraction of images with long TEs from those with short TEs, and subtraction where the long $T_{1}$ long $T_{2}$ fluid is unaffected by the preparation (e.g., MT), as well as diffusion weighting. There are also specific processes that reduce signal from flowing fluids (dephasing). This effect can be increased by the use of gradients, as well as the reduction in signal produced by diffusion in the presence of a gradient. Longer $T_{2}$ signals can also be reduced by dephasing the steady state in bSSFP sequences, and by the use of multiple spokes and nulling for short $T_{1}$ fat in radial acquisitions (69).

There are also other methods including the use of a long $90^{\circ}$ pulse to selectively produce transverse magnetization of longer $\mathrm{T}_{2}$ components followed by dephasing using gradients. This can be regarded as a $T_{2}$ preparation pulse since it selectively reduces signals as a function of their $T_{2} s$.

Inversion pulses alone may fully invert long $\mathrm{T}_{2}$ magnetization but concurrently create transverse magnetization which can be used to image ultrashort of short $\mathrm{T}_{2}$ tissues with fluid signal suppression.

In addition to suppressing signal in these ways it is also possible to use composite pulses to selectively filter the detected signal as a function $\mathrm{T}_{2}$ of tissues or fluids (70). One form of this $\mathrm{T}_{2}$-preparation is a $90^{\circ}-180^{\circ}-90^{\circ}$ pulse to selectively rotate the longitudinal magnetization into the transverse plane, allow transverse relaxation during which short $T_{2}$ components decay to zero and long $T_{2}$ components are largely unchanged. The magnetization is then rotated into the longitudinal direction. The long $\mathrm{T}_{2}$ magnetization is inverted and recovers from $-M_{Z}$, while the short $T_{2}$ magnetization recovers from zero. The long $T_{2}$ can then be nulled and the shorter $T_{2}$ components can be detected.

\section{Nomenclature}

This section seeks to clarify potential points of confusion.

(i) The term attenuation is used generally to describe a complete or partial reduction in signal produced by nulling, saturation, subtraction, or other methods. The term signal suppression is used in the same way. Nulling is specific for attenuation produced by an inversion pulse.

(ii) Tissue $T_{1} s$, and $T_{2} s$ as well as TIs and TEs are described as ultrashort, short, intermediate and long as general descriptive terms with the addition of "supershort" to describe $\mathrm{T}_{2} \mathrm{~s}$ of tissue that are not directly visible with MRI (e.g., $<0.1 \mathrm{~ms}$ ) but which are important in MT. The range of $T_{2} \mathrm{~s}$ is $<0.1$ supershort, $0.1-1$ ultrashort, 1-10 ms short, 10-100 ms medium and 100+ ms long and TEs follow this pattern.

The description of $\mathrm{TI}_{s}$ is approximately TIu (as for $\mathrm{TI}_{\text {WATW }}$ ) $<20 \mathrm{~ms}$, short, $\mathrm{TI}_{\mathrm{s}}$, when the longitudinal magnetization is generally negative (except for fat), as with the STIR sequence, intermediate, $\mathrm{TI}_{\mathrm{i}}$, when tissue magnetization is positive but CSF is negative as is typical for brain imaging with MP-RAGE etc, and long, $\mathrm{TI}_{\mathrm{l}}$, when tissue magnetization is longer again including CSF as zero signal, as with the $T_{2}$-FLAIR sequence. The range of $T_{1}$ values follows this general pattern with ultrashort in blood about $20 \mathrm{~ms}$ following I.V. Gd-DTPA, short about that of fat i.e., $200 \mathrm{~ms}$, intermediate, and long up to that of fluids (e.g., 4,000 $\mathrm{ms}$ for CSF).

(iii) the term fat saturation is used to describe both fat nulling typically with an "inversion" pulse just beyond $90^{\circ}$ as with ultrashort $\mathrm{TI}_{\mathrm{WATN}}$ as well as with CHESS where a $90^{\circ}$ pulse is used to selectively rotate the magnetization and it is then dephased with gradients.

(iv) The term $T_{2}$-FLAIR is used to describe the common CSF nulled form of the sequence as well as another form of the IR sequence in which $\mathrm{T}_{2}$ preparation is used (71). $T_{1}$-FLAIR is a sequence with an intermediate TI in which CSF signal is nulled with the help of a shorter TR than is usually used with a $\mathrm{T}_{2}$-FLAIR sequence (72).

\section{Generalization}

Many of the principles outlined above are applicable to the 20 or more fluids and the 200 or more tissues present in the body.

The emphasis is on features such as: (i) demonstrating normal anatomy including the situation where there are two 
Table 4 TP-Filters in mathematical form

\begin{tabular}{|c|c|c|c|c|}
\hline TP & $\rho_{\mathrm{m}}$ & $\mathrm{T}_{1}$ & $\mathrm{~T}_{2}$ & $D^{*}$ \\
\hline Typical sequence & SE & SE & SE & PGSE \\
\hline $\mathrm{S}_{\mathrm{TP}}$ & $\rho_{\mathrm{m}}$ & $1-e^{-\frac{T R}{T_{1}}}$ & $\mathrm{e}^{-\frac{\mathrm{TE}}{\mathrm{T}_{2}}}$ & $\mathrm{e}^{-\mathrm{bD*}}$ \\
\hline$\frac{\mathrm{S}_{\mathrm{TP}}}{\ln \mathrm{TP}}$ & $\rho_{\mathrm{m}}$ & $-\frac{\mathrm{TR}}{\mathrm{T}_{1}} \mathrm{e}^{-\frac{\mathrm{TR}}{\mathrm{T}_{1}}}$ & $\frac{\mathrm{TE}}{\mathrm{T}_{2}} \mathrm{e}^{-\frac{\mathrm{TE}}{\mathrm{T}_{2}}}$ & $-b D^{*} e^{-b D^{*}}$ \\
\hline
\end{tabular}

*, Neglecting higher order terms.

Table 5 TP-Filters in mathematical form

\begin{tabular}{|c|c|c|c|c|}
\hline TP & $\mathrm{T}_{1}$ & $\mathrm{~T}_{1}$ & $\mathrm{~T}_{1}$ & $\mathrm{~T}_{1}$ \\
\hline$S_{T P}$ & $1-2 e^{-\frac{T I}{T_{1}}}$ & $\begin{array}{l}1-2 \mathrm{e}^{-\frac{\mathrm{TI}}{\mathrm{T}_{1}}}+\mid 1-2 \mathrm{e}^{-\frac{\mathrm{TI}}{\mathrm{T}_{1}} \mid} \\
\text { For } \mathrm{T}_{1} \geq \mathrm{T}_{1} \mathrm{sp}=0 \\
\text { For } \mathrm{T}_{1} \leq \mathrm{T}_{1} \mathrm{sp}=1-2 \mathrm{e}^{-\frac{\mathrm{TI}}{\mathrm{T}_{1}}}\end{array}$ & $\begin{array}{l}1-2 \mathrm{e}^{-\frac{\mathrm{TI}}{\mathrm{T}_{1}}}-\mid 1-2 \mathrm{e}^{-\frac{\mathrm{TI}}{\mathrm{T}_{1}} \mid} \\
\text { For } \mathrm{T}_{1} \leq \mathrm{T}_{1} \mathrm{sp}=0 \\
\text { For } \mathrm{T}_{1} \geq \mathrm{T}_{1} \mathrm{sp}=1-2 \mathrm{e}^{-\frac{\mathrm{TI}}{\mathrm{T}_{1}}}\end{array}$ & $\begin{array}{l}1-2 \mathrm{e}^{-\frac{\mathrm{Tl}_{1}}{\mathrm{~T}_{1}}}-\mid 1-2 \mathrm{e}^{-\frac{\mathrm{TI}}{\mathrm{T}_{1}} \mid} \\
+2 \mathrm{e}^{-\frac{\mathrm{TI}_{2}}{\mathrm{~T}_{1}}}+\left|1-2 \mathrm{e}^{-\frac{\mathrm{TI} \mathrm{I}_{2}}{\mathrm{~T}_{1}}}\right| \\
\text { For } \mathrm{T}_{1} \leq \mathrm{T}_{1} \mathrm{sp}_{1}=0 \\
\text { For } \mathrm{T}_{1} \geq \mathrm{T}_{1} \mathrm{sp}_{2}=0 \\
\text { For } \mathrm{T}_{1} \mathrm{sp}_{1} \leq \mathrm{T}_{1} \leq \mathrm{T}_{1} \mathrm{sp}_{2}=1-2 \mathrm{e}^{-\frac{\mathrm{TI}}{\mathrm{T}_{1}}}\end{array}$ \\
\hline$\frac{\partial \mathrm{S}_{\mathrm{TP}}}{\partial \ln \mathrm{TP}}$ & $-2 \frac{\mathrm{TI}}{\mathrm{T}_{1}} \mathrm{e}^{-\frac{\mathrm{TI}}{\mathrm{T}_{1}}}$ & $\begin{array}{l}\text { For } \mathrm{T}_{1} \leq \mathrm{T}_{1} \mathrm{sp} \\
-2 \frac{\mathrm{TI}}{\mathrm{T}_{1}} \mathrm{e}^{-\frac{\mathrm{TI}}{\mathrm{T}_{1}}}\end{array}$ & $\begin{array}{l}\text { For } \mathrm{T}_{1} \geq \mathrm{Tsp} \\
-2 \frac{\mathrm{TI}}{\mathrm{T}_{1}} \mathrm{e}^{-\frac{\mathrm{TI}}{\mathrm{T}_{1}}}\end{array}$ & $\begin{array}{l}\text { For } \mathrm{T}_{1} \mathrm{sp}_{1} \leq \mathrm{T}_{1} \leq \mathrm{T}_{1} \mathrm{sp}_{2} \\
-2 \frac{\mathrm{TI}}{\mathrm{T}_{1}} \mathrm{e}^{-\frac{\mathrm{TI}}{\mathrm{T}_{1}}}+2 \mathrm{e}^{-\frac{\mathrm{TI}_{2}}{\mathrm{~T}_{1}}}\end{array}$ \\
\hline
\end{tabular}

*, Neglecting higher order terms. $T_{1} s p, T_{1}$ specific; $T_{1} s p_{1}$, First $T_{1}$ specific; $T_{1} s p_{2}$, Second $T_{1}$ specific.

(or more) well defined normal tissue types (e.g., gray and white matter, cortex and medulla); (ii) distinguishing normal and abnormal tissue, and (iii) demonstrating specific tissues or fluids (e.g., ultrashort $\mathrm{T}_{2}$ tissue components).

It may be useful to package groups of MASTIR sequences with different options for particular applications e.g., for the brain with sequences to show high synergistic $T_{1}$ contrast, high synergistic $T_{1}$ and $T_{2}$ contrast, high synergistic $T_{1}$, $\mathrm{T}_{2}$ and $\mathrm{D}^{*}$ contrast, and contrast enhancement using a synergistic $T_{1}$ sequence, as well as the presence of iron, myelin, myelin water, blood and other fluids.
The basic components of MASTIR sequences are available on many commercial MR systems in the form of DIR, MP-RAGE, MP-2RAGE, IR-UTE/ZTE, DX, diffusion weighted, MT, angiography and perfusion sequences, and these are often in time efficient form. As a result, the entry cost for implementing MASTIR sequences may be quite low, although effort is usually required to optimize contrast and spatial resolution for particular applications, control artefacts and achieve further time efficiency. Many of the TP-filters discussed in this paper using a graphic format are shown in mathematical form in Tables 4,5,6. 
Table 6 TP-Filters in mathematical form

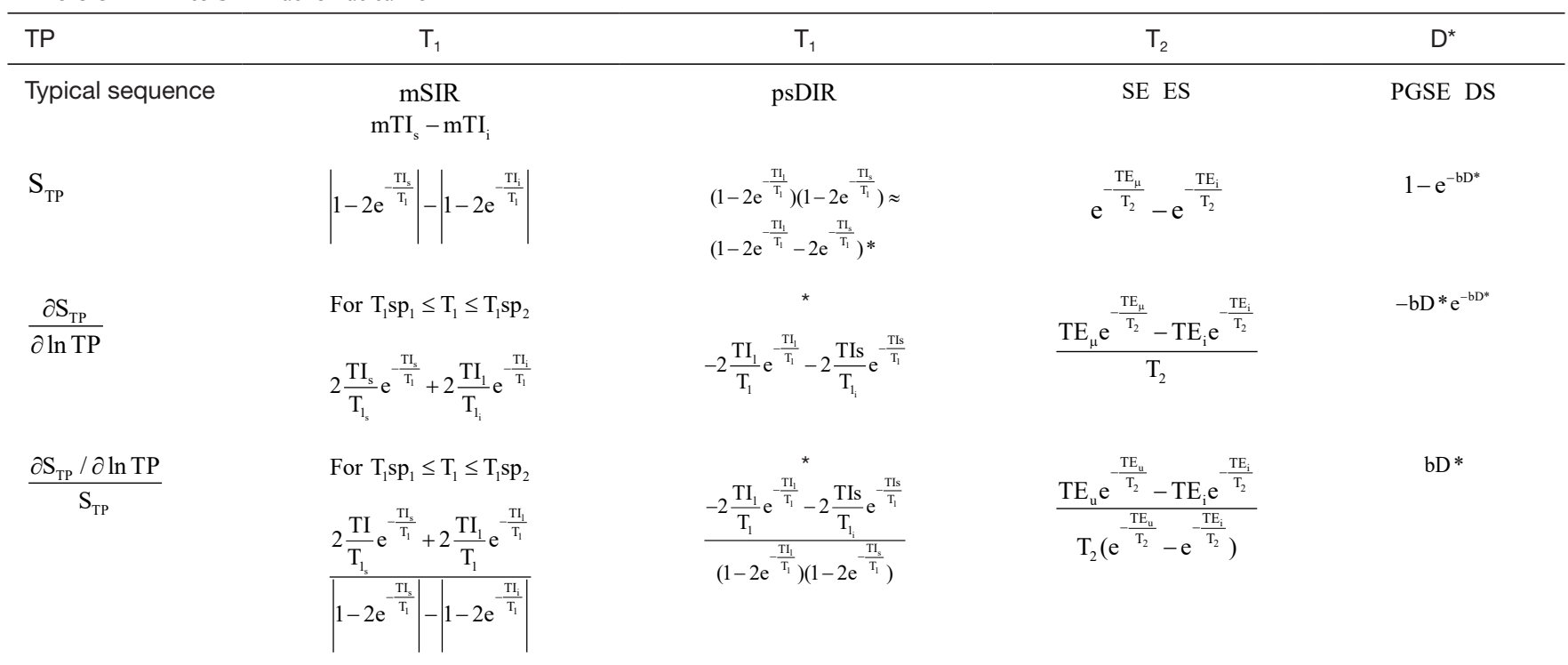

${ }^{*}$, Neglecting higher order terms. $T_{1} s p, T_{1}$ specific; $T_{1} s_{1}$, First $T_{1}$ specific; $T_{1} s p_{2}$, Second $T_{1}$ specific.

\section{Acknowledgments}

Funding: This study was supported by the National Institutes of Health (R01NS092650).

\section{Footnote}

Conflicts of Interest: All authors have completed the ICMJE uniform disclosure form (available at http://dx.doi. org/10.21037/qims-20-568). JD serves as an unpaid editorial board member of Quantitative Imaging in Medicine and Surgery. The other authors have no conflicts of interest to declare.

Open Access Statement: This is an Open Access article distributed in accordance with the Creative Commons Attribution-NonCommercial-NoDerivs 4.0 International License (CC BY-NC-ND 4.0), which permits the noncommercial replication and distribution of the article with the strict proviso that no changes or edits are made and the original work is properly cited (including links to both the formal publication through the relevant DOI and the license). See: https://creativecommons.org/licenses/by-nc-nd/4.0/.

\section{References}

1. Doyle FH, Gore JC, Pennock JM, Bydder GM, Orr JS, Steiner RE, Young IR, Burl M, Clow H, Gilderdale DJ,
Bailes DR, Walters PE. Imaging of the brain by nuclear magnetic resonance. Lancet 1981;2:53-7.

2. Smith FW, Mallard JR, Reid A, Hutchison JM. Nuclear magnetic resonance tomographic imaging in liver disease. Lancet 1981;1:963-6.

3. Young IR, Hall AS, Pallis CA, Legg NJ, Bydder GM, Steiner RE. Nuclear magnetic resonance imaging of the brain in multiple sclerosis. Lancet 1981;2:1063-6.

4. Bydder GM, Young IR. Clinical use of the inversion recovery sequence. J Comput Assist Tomogr 1985;9:659-75.

5. Redpath TW, Smith FW. Technical note: use of a double inversion recovery pulse sequence to image selectively gray or white brain matter. Br J Radiol 1994;67:1258-63.

6. Zee CS, Segall HD, Ferk MR. SPIR MRI in spinal diseases. J Comput Assist Tomogr 1992;16:356-60.

7. De Coene B, Hajnal JV, Gatehouse P, Longmore DB, White SJ, Oatridge A, Pennock JM, Young IR, Bydder GM. MR of the brain using fluid-attenuated inversion recovery (FLAIR) pulse sequences. AJNR Am J Neuroradiol 1992;13:1555-64.

8. IT'IS Foundation. IT'IS database for thermal and electromagnetic parameters of biological tissues. V4.0 May 15 2018. doi:10.13099/VIP21000-04-0.

9. McRobbie DW, Moore EA, Graves MJ, Prince MR. MRI from Picture to Proton. 3rd ed. Cambridge: Cambridge University Press 2016, p.131.

10. Wikipedia. The Free Encyclopedia. Available online: 
https://en.wikipedia.org/w/index.php? Title=Relaxation (NMR)\&oldid=921129080

11. Haase A, Frahm J, Hänicke W, Matthaei D. 1H NMR chemical shift selective (CHESS) imaging. Phys Med Biol 1985;30:341-4.

12. Young IR, Szeverenyi NM, Du J, Bydder GM. Pulse sequences as tissue property filters (TP-filters): a way of understanding the signal, contrast and weighting of magnetic resonance images. Quant Imaging Med Surg 2020;10:1080-120.

13. Ma Y, Zhao W, Searleman A, Du J, Szeverenyi NM, Bydder GM. Use of entwined magnitude and phasesensitive inversion REcovery (EMPIRE) pulse sequences to study the brain and knee. ISMRM, 2018, Paris, France.

14. Fan S, Ma Y, LvX, Du J, Bydder GM, Szeverenyi NM. Demonstration of abnormal cortical layers in Alzheimer's disease using subtracted tissue attenuated inversion (STAIR) pulse sequences. ISMRM, 2362, 2017, Honolulu, USA.

15. Ma Y, Zhao W, Searleman A. Cortical bone imaging using an Ultrashort Echo Time (UTE) phase sensitive clinical inversion recovery subtraction technique. ISMRM 2018:5166.

16. Specter H, Bido J, Henandez G, Rivera D, Suazo L, Valenzuela S, Fermin R, Oviedo J, Foerster B, Gonzalez $\mathrm{C}$, Stoeter P. Inversion recovery sequences improve delineation of optic pathways in the proximity of supratellar lesions. J Radiosurg SBRT 2018;5:115-22.

17. Kim JH, Ryu JK, Jahng GH, Song JY. Double inversion recovery MR imaging of the breast: efficacy in detection of breast cancer. J Magn Reson Imaging 2014;39:51-8.

18. Greenman RL, Shirosky JE, Mulkern RV, Rofsky NM. Double inversion black-blood fast spin echo imaging of the human heart: a comparison between 1.5 and 3.0T. J Magn Reson Imaging 2003;17:648-55.

19. Simonetti OP, Finn JP, White RD, Laub G, Henry DA. "Black Blood" T2-weighted inversion recovery MR imaging of the heart. Radiology 1996;199:49-57.

20. Dong HZ, Worters PW, Wu HH, Ingle RR, Vasanawala SS, Nishimura DG. Non-contrast enhanced renal angiography using multiple inversion recovery and alternating TR balanced steady state free precession. Magn Reson Med 2013;70:527-36.

21. Andia ME, Botnar RM. Arterial spin labeling angiography using a triple inversion recovery prepulse. Magn Reson Med 2012;67:477-483.

22. Yarnykh VL, Yuan C. T1-insensitive flow suppression using quadruple inversion-recovery. Magn Reson Med
2002;48:899-905.

23. Atanasova IP, Kim D, Lim RP, Storey P, Kim S, Guo H, Lee VS. Noncontrast MR angiography for comprehensive assessment of abdominopelvic arteries using quadruple inversion-recovery preconditioning and 3D balanced steady-state free precession imaging. J Magn Reson Imaging 2011;33:1430-9.

24. Mugler JP, Brookeman JR. Three-dimensional magnetization - prepared rapid gradient-echo imaging (3D MP RAGE). Magn Reson Med 1990;15:152-7.

25. Marques JP, Kober T, Krueger G, van der Zwaag W, Van de Moortele PF, Gruetter R. MP2RAGE, a self bias-field corrected sequence for improved segmentation and T1mapping at high field. NeuroImage 2010;49:1271-81.

26. Marques JP, Gruetter R. New developments and applications of the MP2RAGE sequence - focusing the contrast and high spatial resolution R1 mapping. PLOS One 2013;8:e69294.

27. Tanner M, Gambarota G, Kober T, Krueger G, Erritzoe D, Marques JP, Newbould R. Fluid and white matter suppression with the MP2RAGE sequence. J Magn Reson Imaging 2012;35:1063-70.

28. Sethi V, Yousry TA, Muhlert N, Ron M, Golay X, Wheeler-Kingshott C, Miller DH, Chard DT. Improved detection of cortical MS lesions with phase-sensitive inversion recovery MRI. J Neurol Neurosurg Psychiatry 2012;83:877-82.

29. Mougin O, Abdel-Fahim R, Dineen R, Pitiot A, Evangelou $\mathrm{N}$, Gowland P. Imaging gray matter with concomitant null point imaging from the phase-sensitive inversion recovery sequence. Magn Reson Med 2016;76:1512-6.

30. Kecskemeti S, Samsonov A, Hurley SA, Dean CD, Field A, Alexander AL. MPnRAGE: a technique to simultaneously acquire hundreds of differently contrasted MP-RAGE images with applications to quantitative T1 mapping. Magn Reson Med 2016;75:1040-53.

31. Ma YJ, Searleman AC, Jang H, Wong J, Chang EY, CoreyBloom J, Bydder GM, Du J. Whole-brain myelin imaging using a 3D Double Echo Sliding Inversion REcovery Ultrashort Echo Time (DESIRE UTE) MRI. Radiology 2020;294:362-74.

32. Wiggermann V, Hernández-Torres E, Traboulsee A, Li DK, Rauscher A. FLAIR2: A combination of FLAIR and T2 for improved MS lesion detection. AJNR 2016;37:259-65.

33. Essig M, Deimling M, Hawighorst H, Debus J, van Kaick G. Assessment of cerebral gliomas by a new dark fluid sequence, High Intensity REduction (HIRE): a 
preliminary study. J Magn Reson Imaging 2000;11:506-17.

34. Madhuranthakam AJ, Lee KS, Yassin A, Brittain JH, Pedrosa I, Rofsky NM, Alsop DC. Improved short tau inversion recovery (iSTIR) for increased tumor conspicuity in the abdomen. MAGMA 2014;27:245-55.

35. Takahara T, Imai Y, Yamashita T, Yasuda S, Nasu S, Van Cauteren M. Diffusion weighted whole body imaging with background body signal suppression (DWIBS): technical improvement using free breathing, STIR and high resolution 3D display. Radiat Med 2004;22:275-82.

36. Goldfarb JW. Magnetic resonance separation imaging using a Divided Inversion Recovery Technique (DIRT). Magn Reson Med 2010;63:1007-14.

37. Park J, Park S, Kim EY, Suh JS. Phase-sensitive, dualacquisition, single-slab, 3D, turbo-spin-echo pulse sequence for simultaneous T2-weighted and fluidattenuated whole-brain imaging. Magn Reson Med 2010;63:1422-30.

38. Jethwa K, AphiwaHanasumet K, Mougin O, Bowtell R, Auer D, Gowland P. Phase enhanced PSIR T1-weighted imaging improves contrast resolution of the nucleus basalis of Meynert at 7T: a preliminary study. Magn Reson Imaging 2019;61:296-9.

39. Piechnik SK, Ferreira VM, Dall'Armellina E, Cochlin LE, Greiser A, Neubauer S, Robson MD. Shortened Modified Look-Locker Inversion recovery (ShMOLLI) for clinical myocardial T1-mapping at 1.5 and $3 \mathrm{~T}$ within a 9 heartbeat breathhold. J Cardiovasc Magn Reson 2010;12:69.

40. Bley TA, Wieben O, François CJ, Brittain JH, Reeder SB. Fat and water magnetic resonance imaging. J Magn Reson Imaging 2010;31:4-18.

41. Shiehmorteza M, Yokoo T, Hamilton G, Bydder M, Rodriguez M, Lavine JE. Hepatic fat quantification in children using multi-echo imaging and fat spectral modeling at 1.5 T. Proc. Intl. Soc. Mag. Reson. Med. 2009:204.

42. Hajnal JV, Baudouin CJ, Oatridge A, Young IR, Bydder GM. Design and implementation of magnetization transfer pulse sequences for clinical use. J Comput Assist Tomogr 1992;16:7-18.

43. Meara SJP, Barker GJ. Impact of incidental magnetization transfer effects on inversion-recovery sequences that use a fast spin-echo readout. Magn Reson Med 2007;58:825-9.

44. Shin W, Gu H, Yang Y. Incidental magnetization transfer contrast by fat saturation preparation pulses in multislice Look-Locker echo planar imaging. Magn Reson Med 2009;62:520-6.

45. Girard OM, Du J, Agemy L, Sugahara KN, Kotamraju
VR, Ruoslahti E, Bydder GM, Mattrey RF. Optimization of iron oxide nanoparticle detection using ultrashort echo time pulse sequences: comparison of T2, T2*, and synergistic T1 - T2* contrast mechanisms. Magn Reson Med 2011;65:1649-60.

46. Nofiele JT, Cheng HL. Ultrashort echo time for improved positive-contrast manganese-enhanced MRI of cancer. PloS One 2013;8:e58617.

47. Sati P, George IC, Shea CD, Gaitán MI, Reich DS. FLAIR*: A combined MR contrast technique for visualising white matter lesions and parenchymal veins. Radiology 2012;265:926-32.

48. Dixon WT. Simple proton spectroscopic imaging. Radiology 1984;153:189-94.

49. Ma J. Dixon techniques for water and fat imaging. J Magn Reson Imaging 2008;28:543-58.

50. Sampath SC, Sampath SC, Bredella MA, Cypess AM, Torriani M. Imaging of Brown Adipose Tissue: State of the Art. Radiology 2016;280:4-19.

51. Karampinos DC, Weidlich D, Wu M, Hu HH, Franz D. Techniques and applications of magnetic resonance imaging for studying brown adipose tissue morphometry and function. Handb Exp Pharmacol 2019;251:299-324.

52. Hu HH, Nayak KS. Change in the proton of T1 of fat and water in mixture. Magn Reson Med 2010;63:494-501.

53. Hamilton G, Smith DL, Bydder M, Nayak KS, Hu HH. MR properties of brown and white adipose tissues. J Magn Reson Imaging 2011;34:468-73.

54. Holstila M, Virtanen KA, Grönroos TJ, Laine J, Lepomäki V, Saunavaara J, Lisinen I, Komo M, Hannukainen JC, Nuutila P, Parkkola R, Borra RJ. Measurement of brown adipose tissue mass using a novel dual-echo magnetic resonance imaging approach: a validation study. Metabolism 2013;62:1189-98.

55. Du J, Takahashi AM, Bae WC, Chung CB, Bydder GM. Dual inversion recovery, ultrashort echo time (DIR UTE) imaging: creating high contrast for short-T(2) species. Magn Reson Med 2010;63:447-55.

56. Wheaton AJ, Miyazaki M. Non-contrast enhanced MR angiography: physical principles. J Magn Reson Imaging 2012;36:286-304.

57. Miyazaki M, Akahane M. Non-contrast enhanced MR angiography: established techniques. J Magn Reson Imaging 2012;35:1-19.

58. Petcharunpaisan S, Ramalho J, Castillo M. Arterial spin labeling in neuroimaging. World J Radiol 2010;2:384-398.

59. Martirosian P, Boss A, Schraml C, Schwenzer NF, Graf H, Claussen CD, Schick F. Magnetic resonance perfusion 
imaging without contrast media. Eur J Nucl Med Mol

Imaging 2010;37:S52-64.

60. Kim SG. Quantification of relative cerebral blood-flow change by Flow-sensitive Alternating Inversion -Recovery (FAIR) technique-application to functional mappying. Magn Reson Med 1995;34:293-301.

61. Wengler K, Bangiyev L, Canli T, Duong TQ, Schweitzer ME, He X. 3D MRI of whole-brain matter permeability with Intrinsic Diffusivity Encoding of Arterial Labeled Spin (IDEALS). NeuroImage 2019;189:401-14.

62. Lu H, van Zijl PCM. A review of the development of vascular-space occupancy (VASO) fMRI. NeuroImage 2012;62:736-742.

63. Huber L, Ivanov D, Handwerker DA, Marrett S, Guidi M, Uludag K, Bandettini PA, Poser BA. Techniques for blood volume fMRI with VASO: from low-resolution mapping towards sub-millimeter layer-dependent applications. NeuroImage 2018;164:131-43.

64. Ma D, Gulani V, Seiberlich N, Liu K, Sunshine JL, Duerk JL, Griswold MA. Magnetic Resonance fingerprinting. Nature 2013;495:187-92.

65. Weigel M, Bien O. Spinal cord imaging using averaged magnetization inversion recovery acquisitions. Magn Reson Med 2018;79:1870-81.

66. Muscogiuri G, Rehwald WG, Schoepf UJ, Suranyi P, Litwin SE, De Cecco CN, Wichmann JL, Mangold S, Caruso D, Fuller SR, Bayer Nd RR, Varga-Szemes A. T1rho and magnetization transfer and Inversion Recovery
(TRAMINER) - prepared imaging: a novel contrastenhanced flow-independent dark-blood technique for the evaluation of myocardial late Gadolinium enhancement in patients with myocardial infarction. J Magn Reson Imaging 2017:45:1429-37.

67. Hwang T-L, van Zijl PCM, Garwood M. Asymmetric adiabatic pulses for $\mathrm{NH}$ selection. J Magn Reson 1999;138:173-7.

68. Ma YJ, Jerban S, Jang H, Chang EY, Du J. Fat suppression for ultrashort echo time imaging using a novel softhand composite radiofrequency pulse. Magn Reson Med 2019;82:2178-87.

69. Carl M, Bydder GM, Du J. UTE imaging with simultaneous water and fat signal suppression using a timeefficient multispoke inversion recovery pulse sequence. Magn Reson Med 2016;76:577-82.

70. Vidarsson L, Cunningham C, Gold GE, Pauly JM. T2selective magnetization preparation pulses. IEEE Trans Med Imaging 2007;26:981-9.

71. Wong EC, Liu TT, Luh VM, Frank LR, Buxton RB. T1 and T2 selective method for improved SNR in CSF attenuated imaging: T2 FLAIR. Magn Reson Med 2001;45:529-32.

72. Melhem ER, Israel DA, Eustace S, Jara H. MR of the spine with fast T1-weighted Fluid-Attenuated Inversion Recovery sequence. AJNR Am J Neuroradiol 1997;18:447-54.
Cite this article as: Ma YJ, Fan S, Shao H, Du J, Szeverenyi NM, Young IR, Bydder GM. Use of Multiplied, Added, Subtracted and/or FiTted Inversion Recovery (MASTIR) pulse sequences. Quant Imaging Med Surg 2020;10(6):1334-1369. doi: 10.21037/qims-20-568 


\section{Appendix: Abbreviations}

\section{General}

CNR: Contrast to Noise Ratio

FDG: FluoroDeoxyGlucose

fMRI: functional MRI

Gd-DTPA: Gadolinium-DethyleneTriaminePentaacetic

Acid

$\mathrm{m}$ : Slope of a curve

mpMRI: multi-parametric MRI

MRI: Magnetic Resonance Imaging

Mxy: transverse Magnetization

Mz: longitudinal Magnetization

p: ratio of inversion times

PET: Positron Emission Tomography

S: Signal

$\mathrm{S} \rho_{\mathrm{m}}$ : Signal from $\rho_{\mathrm{m}}$ (mobile proton density) component of pulse sequence

$\mathrm{S}_{\mathrm{T} 1}$ : Signal from $\mathrm{T}_{1}$ component of pulse sequence

$\mathrm{S}_{\mathrm{T} 2}$ : Signal from $\mathrm{T}_{2}$ component of pulse sequence

$\mathrm{S}_{\mathrm{D}^{*}}$ : Signal from Diffusion component of pulse sequence

SNR: Signal to Noise Ratio

sc MRI: synergistic contrast MRI

$\theta$ : phase

$\theta_{\mathrm{TE} 1}-\theta_{\mathrm{TE} 2}:$ phase at first echo time minus phase at second echo time

\section{Tissues and Fluids}

B: Blood

BAT: Brown Adipose Tissue

CSF: Cerebrospinal Fluid

F: Fat

G: Gray Matter

W: White Matter

WAT: White Adipose Tissue

\section{Tissue and Fluid MR Properties}

CBV: Cerebral Blood Volume

D: Diffusion

D*: Apparent Diffusion Coefficient

MT: Magnetization Transfer

MTR: Magnetization Transfer Ratio

$\rho_{\mathrm{m}}$ : Mobile Proton Density

PDFF: Proton Density Fat Fraction

$\mathrm{T}_{1}$ : Longitudinal Relaxation Time

$\mathrm{T}_{\text {1sp } 1}$ : Longitudinal Relaxation Time Specific 1
$\mathrm{T}_{\text {1sp2: }}$ Longitudinal Relaxation Time Specific 2

$\mathrm{T}_{2}$ : Transverse Relaxation Time

\section{Pulse Sequences}

AIR: Added Inversion Recovery

AIRES: Added Inversion Recovery Echo Subtraction

ASL: Arterial Spin Labelling

CHESS: Chemical Shift Selective Saturation

DESIRE: Double Echo Sliding Inversion REcovery

DIR: Double Inversion Recovery

DS: Diffusion Subtraction

DX: Dixon

EPI: Echo Planar Imaging

ES: Echo Subtraction

FAIR: Flow-sensitive Alternating Inversion Recovery

FLAIR: FLuid Attenuated Inversion Recovery

FLAIR $^{2}$ : Combined FLAIR and $\mathrm{T}_{2}$ imaging

FLAIR : Combined FLAIR and $\mathrm{T}_{2}{ }^{*}$ imaging

FLN: FLuid Nulled

FSE: Fast Spin Echo

FSSIR: Fat Suppressed Subtraction Inversion Recovery

FWSS: Fat Water Suppression and/or Separation

GE: Gradient Echo

IDEAL: Iterative Decomposition of water and fat with

Echo Asymmetry and Least: : squares estimation

IDEALS: Intrinsic Diffusivity Encoding of Arterial Labeled Spin

IR: Inversion Recovery

IRES: Inversion Recovery Echo Subtraction

LIPO-QUANT: Liver Imaging of Phase-interference

related signal Oscillation and QUANTification

MTS: Magnetization Transfer Subtraction

MASTIR: Multiplied, Added, Subtracted and/or fiTted

Inversion Recovery

MIR: Multiplied Inversion Recovery

MIRES: Multiplied Inversion Recovery Echo Subtraction

$\left(\mathrm{mTI}_{\mathrm{s}} \mathrm{x} \mathrm{mTI}_{\mathrm{s}}\right)\left(\mathrm{TE}_{\mathrm{u}}-\mathrm{TE}_{\mathrm{s}}\right)$

MP-RAGE: Magnetization Preparation - Rapid Acquisition

with Gradient Echo

MP-2RAGE: Magnetization Preparation - 2 Rapid

Acquisition with Gradient Echo

MPnRAGE: Magnetization Preparation n Rapid

Acquisition with Gradient Echo

MSIR: Multiplied Subtracted Inversion Recovery

r: reversed

rSIR: reverse Subtraction Inversion Recovery $\left(\mathrm{mTI}_{\mathrm{i}}-\mathrm{mTI}_{\mathrm{i}}\right)$

QIR: Quadruple Inversion Recovery 
SAIR: Subtracted Added Inversion Recovery

SAIRES: Subtracted Added Inversion Recovery Echo

Subtraction

SE: Spin Echo

SENSE: SENSitivity Encoding

ShMOLLI: Shortened MOdified Look-Locker Inversion

recovery

SIR: Subtracted Inversion Recovery $\left(\mathrm{mTI}_{\mathrm{s} / \mathrm{i} /}-\mathrm{mTI}_{\mathrm{s} / \mathrm{i} /}\right)$

SIRDES: Subtracted Inversion Recovery Diffusion Echo

Subtraction

SIRES: Subtracted Inversion Recovery Echo Subtraction

SIRGES: Subtraction Inversion Recovery Gradient Echo

Subtraction

SIRDGES: Subtraction Inversion Recovery Diffusion

Gradient Echo Subtraction

SPAIR: SPectral Adiabatic Inversion Recovery/SPectral

Attenuated Inversion Recovery

SPECIAL: SPECtral Inversion At Lipid

SPIR: SPectral Inversion Recovery/Spectral Presaturation

Inversion Recovery

STAIR: Short TR Adiabatic pulse prepared Inversion

Recovery

STAIRES: Short TR Adiabatic pulse prepared Inversion

Recovery Echo Subtraction

STIR: Short Inversion Time Inversion Recovery

STIRES: Short Inversion Time Inversion Recovery with

Echo Subtraction

$\mathrm{S}^{1}$ IR: Subtracted Inversion Recovery $\left(\mathrm{psIR}_{\mathrm{s} / \mathrm{i} / 1}-\mathrm{mIR}_{\mathrm{s} / \mathrm{i} / \mathrm{I}}\right)$

S$^{2} \mathrm{IR}$ : Subtracted S IR $\left\{\left(\mathrm{mTI}_{\mathrm{s} / \mathrm{i}}-\mathrm{psTI}_{\mathrm{s} / \mathrm{i}}\right)-\left(\mathrm{mTI}_{1}-\mathrm{psTI}_{1}\right)\right\}$

TIR: Triple Inversion Recovery

UTE: Ultrashort Echo Time

VSO: Vascular Space Occupancy

ZTE: Zero Echo Time

\section{Pulse Sequence Parameters}

ASE: Asymmetric Spin Echo

b: diffusion sensitivity parameter

dc: data collection

D o - D w: Diffusion without (o) minus Diffusion with (w) sensitization

$\mathrm{m}$ : magnitude (reconstruction)

MP: Magnetization Preparation

MTo - MTw: Magnetization Transfer without (o) minus

Magnetization Transfer with (w) sensitization

NPI: Null Point Imaging

ps: phase-sensitive (reconstruction)

rf: radiofrequency

SENSE: SENSitivity Encoding

TI: Inversion Time

TIi: intermediate Inversion Time

TIl: long Inversion Time

TIs: short Inversion Time

TIu: ultrashort Inversion Time

TE: Echo Time

TEi: intermediate Echo Time

$\mathrm{TE}_{\mathrm{in}}$ : in-phase Echo Time

$\mathrm{TE}_{\text {out }}$ : out-of-phase Echo Time

TEl: long Echo Time

TEs: short Echo Time

TEu: ultrashort Echo Time

$\mathrm{TE}_{1}$ : first Echo Time

$\mathrm{TE}_{2}$ : second Echo Time

$\mathrm{TI}_{\mathrm{BATN}}$ Inversion Time for Brown Adipose Tissue Nulling

$\mathrm{TI}_{\mathrm{FLN}}$ : Inversion Time for FLuid Nulling

$\mathrm{TI}_{\text {WATN }}$ Inversion Time for White Adipose Tissue Nulling

TR: Repetition Time 